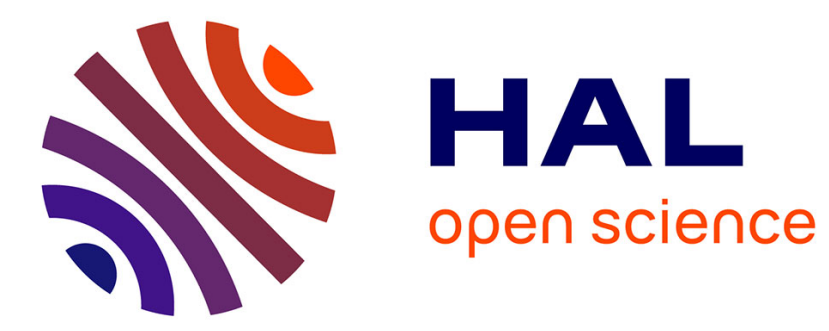

\title{
Logarithmes des points rationnels des variétés abéliennes
}

Vincent Bosser, Eric Gaudron

\section{To cite this version:}

Vincent Bosser, Eric Gaudron. Logarithmes des points rationnels des variétés abéliennes. Canadian Journal of Mathematics, 2019, 71 (2), pp.247-298. 10.4153/CJM-2018-005-7 . hal-02172182

\section{HAL Id: hal-02172182 \\ https://hal.science/hal-02172182}

Submitted on 3 Jul 2019

HAL is a multi-disciplinary open access archive for the deposit and dissemination of scientific research documents, whether they are published or not. The documents may come from teaching and research institutions in France or abroad, or from public or private research centers.
L'archive ouverte pluridisciplinaire $\mathbf{H A L}$, est destinée au dépôt et à la diffusion de documents scientifiques de niveau recherche, publiés ou non, émanant des établissements d'enseignement et de recherche français ou étrangers, des laboratoires publics ou privés. 


\title{
LOGARITHMES DES POINTS RATIONNELS DES VARIÉTÉS ABÉLIENNES
}

\author{
VINCENT BOSSER ET ÉRIC GAUDRON
}

\begin{abstract}
RÉsumé. Nous démontrons une généralisation du théorème des périodes de Masser et Wüstholz où la période est remplacée par un logarithme non nul $u$ d'un point rationnel $p$ d'une variété abélienne définie sur un corps de nombres. Nous en déduisons des minorations explicites de la norme de $u$ et de la hauteur de Néron-Tate de $p$ qui dépendent des invariants classiques du problème dont la dimension et la hauteur de Faltings de la variété abélienne. Les démonstrations reposent sur une construction de transcendance du type Gel'fond-Baker de la théorie des formes linéaires de logarithmes dans laquelle se greffent des formules explicites provenant de la théorie des pentes d'Arakelov.

Abstract. Let $u$ be a logarithm of an algebraic point $p$ of an abelian variety defined over a number field. Let $A_{u}$ be the smallest abelian subvariety whose tangent space at the origin contains $u$. We give a bound for the geometrical degree of $A_{u}$ which generalizes the famous period theorem by Masser and Wüstholz. From this statement, we also deduce some lower bounds for the norm of $u$ and for the Néron-Tate height of $p$. The main new feature of these results is that the bounds are fully explicit in terms of the classical invariants of the problem, among which the dimension and the Faltings height of the abelian variety. The proofs rest on tools from Gel'fond-Baker theory of linear forms in logarithms and from Arakelov slope theory.
\end{abstract}

\section{INTRODUCTION}

1.1. Dans ce texte nous proposons une généralisation du théorème des périodes de Masser et Wüstholz où nous remplaçons la période par un logarithme d'un point rationnel d'une variété abélienne définie sur un corps de nombres. Nous examinons ensuite les conséquences d'un tel énoncé sur la norme du logarithme et la hauteur de Néron-Tate du point rationnel associé en donnant des minorants explicites de ces quantités. Nous montrerons également une version effective d'un théorème de Bertrand portant sur le degré du sous-groupe algébrique minimal contenant un point rationnel donné. Une partie des résultats présentés ici a fait l'objet d'un compte-rendu aux Oberwolfach reports [Ga4].

Soient $k$ un corps de nombres de degré $D$ et $\sigma_{0}: k \hookrightarrow \mathbb{C}$ un plongement complexe. Soit $A$ une variété abélienne définie sur $k$, de dimension $g$, que l'on munit d'une polarisation $L$. La variété abélienne complexe $A_{\sigma_{0}}$ obtenue par extension des scalaires à partir de $A$ possède un espace tangent à l'origine $t_{A_{\sigma_{0}}}$ et une application exponentielle $\exp _{A_{\sigma_{0}}}: t_{A_{\sigma_{0}}} \rightarrow A_{\sigma_{0}}$ surjective. Soient $p \in A(k)$ et $u \in t_{A_{\sigma_{0}}}$ un logarithme de l'image de $p$ dans $A_{\sigma_{0}}$, c'est-à-dire tel que $\exp _{A_{\sigma_{0}}}(u)=\sigma_{0}(p)$. Enfin considérons la plus petite sous-variété abélienne $A_{u}$ de $A_{\sigma_{0}}$ dont l'espace tangent à l'origine contient $u$.

Classiquement un théorème des périodes fournit une estimation du degré géométrique de $A_{u}$ relativement à $L$ lorsque $u$ est une période non nulle de $A_{\sigma_{0}}$ en fonction des invariants de la variété (dimension, hauteur de Faltings, degré de $k$ ) et de la norme de $u$. Ceci correspond donc au cas $p=0$. Le premier énoncé de ce type a été obtenu en 1993 par Masser et Wüstholz [MaWü]. Une version améliorée et entièrement explicite a récemment été démontrée par Gaudron et Rémond [GR2] en s'inspirant de l'approche arakelovienne de Bost [Bo1] et d'un résultat non publié de David [Da].

MSC 2010 : 11J86 (11J95, 11G10, 11G50, 14G40).

Mots-clefs : Théorème des périodes, variété abélienne, logarithme, méthode de Gel'fond-Baker, théorie des pentes, hauteur de Néron-Tate, lemme d'interpolation.

Date: 7 juin 2018. 
Notre premier résultat est un théorème des périodes étendu au logarithme $u$ d'un point $k$-rationnel $p$ quelconque de $A$, appelé théorème des périodes généralisé dans la suite. Nous notons $\|\cdot\|_{L, \sigma_{0}}$ la norme hermitienne sur $t_{A_{\sigma_{0}}}$ induite par la forme de Riemann de $L_{\sigma_{0}}, \widehat{h}_{L}(p)$ la hauteur de NéronTate du point $p$ relative à $L$ et $h_{F}(A)$ la hauteur de Faltings stable de $A$ (voir le début de la partie 3 pour la normalisation choisie).

Théorème 1.1. Avec les données $\left(k, \sigma_{0}, A, L, p, u\right)$ ci-dessus, si $u \neq 0$ et si $g \geq 2$ alors on $a$

$$
\begin{aligned}
\left(\operatorname{deg}_{L} A_{u}\right)^{1 / \operatorname{dim} A_{u} \leq} & (100 g)^{4 g}\left(D \widehat{h}_{L}(p)+\|u\|_{L, \sigma_{0}}^{2}\right) \\
& \times\left(D \max \left\{1, \log D, h_{F}(A), \log \left(D \widehat{h}_{L}(p)+\|u\|_{L, \sigma_{0}}^{2}\right)\right\}\right)^{1+1 / \operatorname{dim} A_{u}} .
\end{aligned}
$$

Si $g=1$ l'inégalité reste vraie avec la constante $1,2.10^{14}$ au lieu de $(100 \mathrm{~g})^{4 g}$.

Le théorème des périodes [GR2, Théorème 1.3] donne un énoncé comparable mais plus précis lorsque $u$ est une période de $A_{\sigma_{0}}$ avec, en particulier, l'exposant du second terme entre parenthèses égal à 1. L'énoncé à la base de ce théorème sera démontré dans la partie 3. D'autres résultats effectifs de nature un peu différente en découlent, comme la minoration suivante de la norme du logarithme $u$.

Théorème 1.2. Si $u \neq 0$ alors on a $\log \|u\|_{L, \sigma_{0}} \geq-(196 g)^{3 g+3} D \max \left\{1, h_{F}(A), \widehat{h}_{L}(p)\right\}$.

Cette estimation, qui peut s'interpréter comme une minoration de formes linéaires en un logarithme sur une variété abélienne, est, à notre connaissance, la première minoration de $\|u\|_{L, \sigma_{0}}$ qui soit entièrement explicite et sans condition sur $p$. Elle est à comparer aux résultats de Pellarin [Pe] et du second auteur [Ga1, Corollaire 1.2]. Si Pellarin propose des exposants légèrement meilleurs pour $h_{F}(A)$ et $\widehat{h}_{L}(p)$, c'est au prix d'hypothèses assez fortes sur $p$ et sa hauteur. De plus il reste une constante non explicitée qui dépend de $g$ et du $\operatorname{degré~} \operatorname{deg}_{L} A$. Ces restrictions ont été supprimées dans [Ga1] sauf l'hypothèse de non torsion modulo les endomorphismes de $A$ du point $p$. Le théorème 1.2 enlève donc toutes ces limitations d'usage.

Une autre conséquence du théorème des périodes généralisé concerne la question de la minoration de la hauteur de Néron-Tate d'un point $p \in A(k)$ qui n'est pas de torsion. Sur ce thème, la littérature s'est surtout concentrée sur la dépendance de la borne en le degré de $k / \mathbb{Q}$ (problème de Lehmer généralisé) ou en la hauteur de $A$ (conjecture de Lang-Silverman). En outre la plupart des énoncés ne concernent que des variétés abéliennes à multiplication complexe. L'énoncé suivant vise au contraire à donner un résultat entièrement explicite avec des hypothèses minimales (voir le paragraphe 4.3 pour des bornes plus précises).

Théorème 1.3. Soit $p \in A(k)$ un point qui n'est pas de torsion. Alors on a

$$
\widehat{h}_{L}(p)^{-1} \leq \max \left\{D+g^{g}, h_{F}(A)\right\}^{6074 g} .
$$

Le groupe de Mordell-Weil $A(k)$ est de type fini et sa partie libre $A(k) / A(k)$ tors est un réseau de $A(k) \otimes_{\mathbb{Z}} \mathbb{R}$ qui, muni de la forme quadratique $p \mapsto \widehat{h}_{L}(p)$, est euclidien. Le théorème s'interprète alors comme une minoration effective du premier minimum de ce réseau. Ceci nous amène à présenter une dernière conséquence du théorème 1.1 qui concerne le sous-groupe algébrique $A_{p}$ engendré par un point $p \in A(k)$. En 1995, Bertrand a montré comment obtenir une borne pour $\left(\operatorname{deg}_{L} A_{p}\right)^{1 / \operatorname{dim} A_{p}}$ linéaire en la hauteur de $p$ lorsque $A_{p}$ est connexe et non nul [Be]. En reprenant ce travail et en y ajoutant un argument de Rémond pour supprimer l'hypothèse de connexité, nous pouvons donner une version totalement explicite du théorème de Bertrand.

Théorème 1.4. Soit $p \in A(k)$ qui n'est pas un point de torsion. Alors on a

$$
\left(\operatorname{deg}_{L} A_{p}\right)^{1 / \operatorname{dim} A_{p}} \leq \widehat{h}_{L}(p)\left((14 g)^{64 g^{2}} D \max \left\{1, \log D, h_{F}(A)\right\}^{2}\right)^{8192 g^{3}} .
$$


1.2. La démonstration du théorème des périodes généralisé 1.1 repose sur une construction de transcendance analogue à celle de [GR2] mais où l'on tient compte des multiples du point $p$. La méthode employée est celle de Gel'fond-Baker de la théorie des formes linéaires de logarithmes dans le canevas de l'approche de Philippon-Waldschmidt [PW]. Nous avons utilisé la méthode de la section auxiliaire [Ga3] dans le cadre de la théorie des pentes [Bo1]. Pour contenir la taille des constantes numériques, nous avons repris et amélioré le lemme d'interpolation analytique de Cijsouw et Waldschmidt [CW] (voir partie suivante), qui était couramment utilisé jusqu'alors. De la méthode de Gel'fond-Baker est issu un énoncé technique (théorème 3.1) qui entraîne les théorèmes 1.1 et 1.2. Le passage à une minoration de la hauteur de $p$, pour obtenir le théorème 1.3, requiert un argument de géométrie des nombres supplémentaire. L'idée est d'appliquer le théorème 1.1 avec un multiple du point $p$ et un logarithme quelconque de ce multiple. Les choix doivent être faits de sorte à minimiser la valeur de la forme quadratique

$$
(\ell, \omega) \mapsto q(\ell, \omega):=\ell^{2} D \widehat{h}_{L}(p)+\|\ell u+\omega\|_{L, \sigma_{0}}^{2}
$$

pour $\ell \in \mathbb{Z}$ et $\omega$ une période de $A_{\sigma_{0}}$. Le premier théorème de Minkowski permet cette optimisation en donnant un majorant de $q(\ell, \omega)$ proportionnel à une puissance fractionnaire de $\widehat{h}_{L}(p)$. Ceci conduit à une minoration de $\widehat{h}_{L}(p)$ qui, bien qu'indépendante de $u$, dépend encore de la polarisation $L$ à travers le degré de $A$. Pour nous en affranchir, nous utilisons alors l'astuce de Zarhin en nous plaçant dans $(A \times \widehat{A})^{4}(\widehat{A}$ est la variété abélienne duale) munie d'une polarisation principale compatible à $L$. Le contrecoup de ce procédé est l'augmentation des constantes numériques puisque la dimension et la hauteur de la variété sont multipliées par huit. Enfin, la démonstration du théorème 1.4 s'appuie encore sur des arguments de géométrie des nombres. Dans son texte [Be],

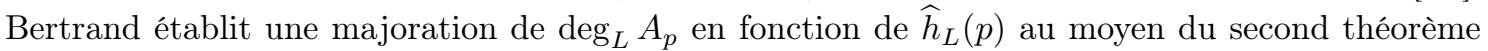
de Minkowski. Les explications détaillées qu'il fournit pour rendre effective la constante ainsi qu'une borne du volume de l'anneau des endomorphismes de $A$ extraite de [Ré1] et le théorème 1.3 permettent aisément d'en déduire une version légèrement plus précise du théorème 1.4 lorsque $A_{p}$ est connexe. Par une méthode que nous a décrite Gaël Rémond, utilisant une borne de l'exposant du groupe de torsion $A(k)_{\text {tors }}$ venant de [Ré1], nous sommes alors en mesure de démontrer le théorème 1.4 dans toute sa généralité.

Remerciements. Nous remercions Daniel Bertrand pour l'échange éclairant que nous avons eu concernant le théorème 1.4 ainsi que Pascal Autissier pour nous avoir signalé une erreur subtile dans une version antérieure de ce texte. Nous rendons grâce également à Gaël Rémond dont les remarques ont permis de corriger et améliorer nombre de détails. Les auteurs ont bénéficié du soutien du projet ANR Gardio 14-CE25-0015.

\section{TABLE DES MATIÈRES}

1. Introduction 1

2. Lemme d'interpolation 3

3. Énoncé clef 6

4. Démonstrations des théorèmes de l'introduction 37

Références 43

\section{LEMME D'INTERPOLATION}

Étant donné une fonction holomorphe $f$ sur un ouvert de $\mathbb{C}$, par lemme d'interpolation nous entendons une borne de la valeur de $f$ en un point de l'ouvert en fonction du supremum de $|f|$ sur un disque contenant ce point et des modules de dérivées de $f$ en des points choisis de l'ouvert. 
Si $r$ est un nombre réel positif et $f$ une fonction définie sur le disque fermé $D(0, r)=\{z \in$ $\mathbb{C} ;|z| \leq r\}$, nous désignons par $|f|_{r}$ le supremum de $|f|$ sur $D(0, r)$. Le résultat principal de cette partie est le suivant.

Proposition 2.1. Soient $S$ un entier strictement positif, $r, R$ deux nombres réels tels que $R>r \geq$ $S-1 / 2$ et $\epsilon \in] 0,1 / 2[$. Soit $f$ une fonction holomorphe dans un ouvert contenant le disque fermé $D(0, R)$. Alors, pour tout entier $T \geq 1$ et tout nombre réel $a>0$, on a

$$
\begin{aligned}
|f|_{r} \leq \frac{R}{R-r} & \left(\frac{r}{R}\right)^{T}\left(\frac{R^{2}\left(r^{2}+\frac{S(2 S-1)}{6}\right)}{R^{4}+r^{2} \frac{S(2 S-1)}{6}}\right)^{T(S-1)} \times|f|_{R} \\
& +\frac{1}{2 \epsilon}\left(\sum_{\substack{|j| \leq S-1 \\
0 \leq \ell \leq T-1}} \frac{\left|f^{(\ell)}(j)\right|}{2^{\ell} \ell !}\right)\left(\frac{r}{a \cos \pi \epsilon}\right)^{T} \max \left\{1, \frac{r}{a}\right\}^{2 T(S-1)}(\operatorname{sh} \pi a)^{T} .
\end{aligned}
$$

L'approche pour la démonstration est classique. Elle repose sur une formule d'interpolation d'Hermite et l'utilisation de facteurs de Blaschke, variante qui, dans ce contexte, remonte (au moins) à un article de Mignotte et Waldschmidt [MiWa]. Une version avec dérivées comme ici a été mise en œuvre (mais non formalisée) par Baker et Wüstholz [BW]. La différence avec l'énoncé de la proposition est le choix des points d'interpolation pour lesquels nous exploiterons la symétrie par rapport à l'origine, comme dans [GR2, § 5].

2.1. Formule d'interpolation d'Hermite. Considérons un entier $n \geq 1$, des nombres complexes $a_{1}, \ldots, a_{n}$ distincts et des entiers strictement positifs $\ell_{1}, \ldots, \ell_{n}$. Soit $z$ un nombre complexe distinct de $a_{1}, \ldots, a_{n}$. Soit $\Gamma$ un lacet fermé délimitant un compact $C$, contenu dans un ouvert $\Omega$ et dont l'intérieur comprend $a_{1}, \ldots, a_{n}, z$. Pour tout $j \in\{1, \ldots, n\}$, soit $\Gamma_{j}$ un contour fermé inclus dans $C$ et dont l'intérieur comprend $a_{j}$ mais pas $a_{1}, \ldots, a_{j-1}, z, a_{j+1}, \ldots, a_{n}$. Soit $\omega: \Omega \rightarrow \mathbb{C}$ une fonction holomorphe qui a pour seuls zéros les $a_{j}$ avec multiplicités $\ell_{j}$. La formule d'interpolation d'Hermite stipule que, pour toute fonction holomorphe $f: \Omega \rightarrow \mathbb{C}$, on a

$$
\frac{f(z)}{\omega(z)}=\frac{1}{2 i \pi} \int_{\Gamma} \frac{f(\xi)}{\omega(\xi)} \frac{\mathrm{d} \xi}{\xi-z}-\frac{1}{2 i \pi} \sum_{j=1}^{n} \sum_{\ell=0}^{\ell_{j}-1} \frac{f^{(\ell)}\left(a_{j}\right)}{\ell !} \int_{\Gamma_{j}} \frac{\left(\xi-a_{j}\right)^{\ell}}{\omega(\xi)} \frac{\mathrm{d} \xi}{\xi-z} .
$$

La démonstration consiste à appliquer le théorème des résidus à la fonction $g(\xi)=f(\xi) /(\omega(\xi)(\xi-$ $z)$ ) le long du contour $\Gamma$. Le résidu de $g$ en $\xi=z$ est $f(z) / \omega(z)$ et $g$ a le même résidu en $a_{j}$ que la fonction

$$
\xi \mapsto \frac{1}{\omega(\xi)(\xi-z)} \sum_{\ell=0}^{\ell_{j}-1} \frac{f^{(\ell)}\left(a_{j}\right)}{\ell !}\left(\xi-a_{j}\right)^{\ell}
$$

qui diffère de $g$ par une fonction holomorphe en $a_{j}$. On exprime alors le résidu de cette fonction au moyen d'une intégrale sur le lacet $\Gamma_{j}$.

2.2. Facteurs de Blaschke. Soient $R>0$ et $a$ un nombre complexe de module $<R$. Le facteur de Blaschke associé au couple $(R, a)$ est la fonction $z \mapsto \frac{R(z-a)}{R^{2}-\bar{a} z}$ qui est holomorphe dans un ouvert contenant $D(0, R)$. Cette fonction est de module constant égal à 1 lorsque $|z|=R$ et, pour $|z| \leq R$, on a

$$
\frac{R|| z|-| a||}{R^{2}-|z||a|} \leq\left|\frac{R(z-a)}{R^{2}-\bar{a} z}\right| \leq \frac{R(|z|+|a|)}{R^{2}+|z||a|} .
$$

Si $S$ est un entier naturel $<R+1$, la fonction

$$
B_{R}(z, S)=\prod_{j=-(S-1)}^{S-1} \frac{R(z-j)}{R^{2}-j z}=\frac{z}{R} \prod_{j=1}^{S-1} \frac{R^{2}\left(z^{2}-j^{2}\right)}{R^{4}-j^{2} z^{2}}
$$


est holomorphe dans un ouvert contenant $D(0, R)$, avec des zéros simples en les entiers compris entre $-(S-1)$ et $S-1$. On a $\left|B_{R}(z, S)\right|=1$ pour tout nombre complexe $z$ de module $R$ et, si $r$ est un nombre réel positif tel que $r \leq R$,

$$
\left|B_{R}(z, S)\right| \leq \frac{r}{R} \prod_{j=1}^{S-1} \frac{R^{2}\left(r^{2}+j^{2}\right)}{R^{4}+r^{2} j^{2}} \quad \text { pour }|z| \leq r .
$$

2.3. En appliquant la formule d'interpolation d'Hermite avec $B_{R}(z, S)^{T}$, nous obtenons le résultat intermédiaire suivant.

Proposition 2.2. Soient $T, S$ des entiers strictement positifs et $r, R$ des nombres réels strictement positifs tels que $R>\max \{r, S-1\}$. Soit $f$ une fonction holomorphe dans un ouvert contenant $D(0, R)$. Alors, pour tout $\epsilon \in] 0,1 / 2[$, pour tout nombre complexe $z$ de module $r$, vérifiant $|z-j| \geq$ $1 / 2$ pour tout $j \in\{-S+1, \ldots, S-1\}$, on a

$$
\begin{aligned}
|f(z)| \leq\left(\frac{r}{R}\right)^{T} & \left(\prod_{j=1}^{S-1} \frac{R^{2}\left(r^{2}+j^{2}\right)}{R^{4}+r^{2} j^{2}}\right)^{T}|f|_{R} \frac{R}{R-r} \\
& +\frac{1}{2 \epsilon}\left(\sum_{\substack{|j| \leq S-1 \\
0 \leq \ell \leq T-1}} \frac{\left|f^{(\ell)}(j)\right|}{2^{\ell} \ell !}\right)\left(\frac{\pi r}{\cos \pi \epsilon}\right)^{T} \prod_{j=1}^{S-1}\left(1+\frac{r^{2}}{j^{2}}\right)^{T} .
\end{aligned}
$$

Démonstration. La formule d'interpolation d'Hermite appliquée avec $\omega=B_{R}(\cdot, S)^{T}$, les cercles $\Gamma=\{\xi \in \mathbb{C} ;|\xi|=R\}$ et $\Gamma_{j}=\{\xi \in \mathbb{C} ;|\xi-j|=1 / 2-\epsilon\}$ et $\ell_{j}=T$ pour tout $j \in\{-S+1, \ldots, S-1\}$ donne

$$
|f(z)| \leq\left|B_{R}(z, S)\right|^{T}|f|_{R} \frac{R}{R-r}+\sum_{|j|<S} \frac{1 / 2-\epsilon}{|z-j|-(1 / 2-\epsilon)} \sum_{\ell=0}^{T-1} \frac{\left|f^{(\ell)}(j)\right|}{2^{\ell} \ell !}\left(\sup _{\xi \in \Gamma_{j}}\left|\frac{B_{R}(z, S)}{B_{R}(\xi, S)}\right|\right)^{T} .
$$

Le terme $\left|B_{R}(z, S)\right|$ a été majoré précédemment. Le quotient devant la dernière somme est plus petit que $1 /(2 \epsilon)$ par hypothèse sur $z$. De plus, pour $\xi \in \Gamma_{j}$, on a

$$
\left|\frac{B_{R}(z, S)}{B_{R}(\xi, S)}\right| \leq \frac{r}{|\xi|} \prod_{h=1}^{S-1}\left(\frac{r^{2}+h^{2}}{\left|\xi^{2}-h^{2}\right|}\right)\left(\frac{\left|R^{4}-\xi^{2} h^{2}\right|}{R^{4}+r^{2} h^{2}}\right) .
$$

Par les points (2) et (4) du lemme 5.2 de [GR2], on a $|\xi| \prod_{h=1}^{S-1}\left|\xi^{2}-h^{2}\right| \geq(S-1) !^{2} \pi^{-1} \cos \pi \epsilon$. En outre, l'appartenance $\xi \in \Gamma_{j}$ implique $|\xi-j| \leq 1 / 2$ donc $\left|\xi^{2}-j^{2}\right| \leq|\xi+j| / 2 \leq|j|+1 / 4$ puis

$$
\left|R^{4}-\xi^{2} h^{2}\right|=\left|R^{4}-j^{2} h^{2}-\left(\xi^{2}-j^{2}\right) h^{2}\right| \leq R^{4}-j^{2} h^{2}+|j| h^{2}+h^{2} / 4 \leq R^{4}+h^{2} / 4 .
$$

Par conséquent, comme $r=|z-0| \geq 1 / 2$, on a $\left|R^{4}-\xi^{2} h^{2}\right| \leq R^{4}+r^{2} h^{2}$ d'où l'on déduit finalement la borne voulue

$$
\left|\frac{B_{R}(z, S)}{B_{R}(\xi, S)}\right| \leq \frac{\pi r}{\cos \pi \epsilon} \prod_{h=1}^{S-1}\left(1+\frac{r^{2}}{h^{2}}\right) .
$$

2.4. Démonstration de la proposition 2.1. Par le principe du maximum il suffit de majorer $|f(z)|$ pour tout nombre complexe $z$ de module $r$. Comme $r \geq S-1 / 2$ on a $|z-j| \geq 1 / 2$ pour tout $j \in\{-S+1, \ldots, S-1\}$ et l'on peut donc appliquer la proposition 2.2 pour majorer $|f(z)|$. L'égalité $\operatorname{sh} \pi a=\pi a \prod_{j=1}^{\infty}\left(1+a^{2} / j^{2}\right)$ et la majoration $1+r^{2} / j^{2} \leq\left(1+a^{2} / j^{2}\right) \max \{1, r / a\}^{2}$ conduisent immédiatement à

$$
\prod_{j=1}^{S-1}\left(1+\frac{r^{2}}{j^{2}}\right) \leq \frac{\operatorname{sh} \pi a}{\pi a} \cdot \max \left\{1, \frac{r}{a}\right\}^{2(S-1)} .
$$


Enfin l'estimation

$$
\prod_{j=1}^{S-1} \frac{r^{2}+j^{2}}{R^{4}+r^{2} j^{2}} \leq\left(\frac{r^{2}+\frac{S(2 S-1)}{6}}{R^{4}+r^{2} \frac{S(2 S-1)}{6}}\right)^{S-1}
$$

découle de la concavité sur $\mathbb{R}^{+}$de la fonction $\xi \mapsto \log \frac{r^{2}+\xi}{R^{4}+r^{2} \xi}$ et de la formule classique pour la somme des carrés d'entiers.

2.5. Remarque. Sur la courbe $\min (|z|, 2|z-1|, 2|z+1|)=1$ tout nombre complexe est de module plus petit que $3 / 2$ et vérifie $|z-j| \geq 1 / 2$ pour tout entier $j$. Ainsi, par le principe du maximum, la proposition 2.2 (avec $r=3 / 2$ et $R=S$ ) donne-t-elle une borne pour $|f|_{1}$ du type

$$
|f|_{1} \leq\left(\frac{3}{2 S} \prod_{j=1}^{S-1} \frac{S^{2}\left((3 / 2)^{2}+j^{2}\right)}{S^{4}+(3 j / 2)^{2}}\right)^{T}|f|_{S} \frac{S}{S-3 / 2}+\frac{S T}{\epsilon} \max _{\substack{|j|<S \\ \ell<T}}\left|\frac{f^{(\ell)}(j)}{2^{\ell} \ell !}\right|\left(\frac{\operatorname{sh}(3 \pi / 2)}{\cos \pi \epsilon}\right)^{T} .
$$

Cette estimation est à comparer à celle de la proposition 5.1 de [GR2]. En particulier le premier terme de droite, à l'intérieur des parenthèses, s'écrit $e^{-2 S(1+o(1))}$ au lieu de $2^{-2 S(1+o(1))}$ comme dans l'énoncé analogue de [GR2].

\section{3. ÉNONCÉ CLEF}

Comme nous l'avons dit dans l'introduction, le théorème des périodes généralisé 1.1 résulte d'un énoncé technique (théorème 3.1) que nous énonçons et démontrons dans cette partie.

Nous reprenons les notations de l'introduction. Considérons donc $k$ un corps de nombres de degré $D \operatorname{sur} \mathbb{Q}$, et fixons un plongement $\sigma_{0}: k \hookrightarrow \mathbb{C}$ de $k$ dans $\mathbb{C}$. Soit $(A, L)$ une variété abélienne polarisée sur $k$, de dimension $g$. Notons $\operatorname{deg}_{L} A$ le degré de $A$ relatif à $L$. Nous désignons par $h(A)$ la hauteur de Faltings stable avec la normalisation de Deligne et $h_{F}(A)=h(A)-(\operatorname{dim} A / 2) \log \pi$ est celle avec la normalisation de Faltings (une définition est rappelée au paragraphe 2.3 de [GR2]). Cette seconde normalisation, qui a l'avantage d'être plus petite que toutes les normalisations usuelles, n'est utilisée que dans l'introduction et dans la partie 4. Soient $t_{A_{\sigma_{0}}}$ l'espace tangent de $A_{\sigma_{0}}$ et $\exp _{A_{\sigma_{0}}}: t_{A_{\sigma_{0}}} \rightarrow A(\mathbb{C})$ son application exponentielle. Soient $p \in A(k)$ et $u \in t_{A_{\sigma_{0}}}$ un logarithme de $\sigma_{0}(p)$. Notons $\widehat{h}_{L}(p)$ la hauteur de Néron-Tate de $p$ et $\|\cdot\|_{L, \sigma_{0}}$ la norme hermitienne sur $t_{A_{\sigma_{0}}}$ induite par $L_{\sigma_{0}}$. L'énoncé clef de cette partie est le suivant :

Théorème 3.1. Soit $E \geq e$ un nombre réel. Supposons que u n'appartienne à l'espace tangent d'aucune sous-variété abélienne stricte de $A_{\sigma_{0}}$. Alors on a :

$$
\begin{aligned}
\left(\operatorname{deg}_{L} A\right)^{1 / g} \log E \leq & c_{0}(g)\left(D \widehat{h}_{L}(p)+\frac{\pi}{2} E^{2}\|u\|_{L, \sigma_{0}}^{2}\right) \\
& \times \max \left\{1, \frac{D}{\log E}, \frac{D}{\log E} \log \frac{D}{\log E}, \frac{D h(A)}{\log E}, \frac{D \log \operatorname{deg}_{L} A}{\log E}\right\}^{1+1 / g}
\end{aligned}
$$

où $c_{0}(1)=8,3.10^{8}$ et $c_{0}(g)=3.10^{6} g^{2 g+11}(\log g)^{2}$ si $g \geq 2$.

La démonstration de ce théorème s'inscrit dans une démarche typique de la transcendance. Elle est issue de la méthode mise au point par Philippon et Waldschmidt [PW] pour obtenir des minorations de formes linéaires de logarithmes dans les groupes algébriques commutatifs définis sur $\overline{\mathbb{Q}}$, méthode dans laquelle se greffent des calculs explicites de la théorie des pentes de Bost [Bo1]. Elle comporte plusieurs étapes. L'idée de base est de construire une section auxiliaire non nulle du faisceau inversible $L^{\otimes n}$ (pour un certain entier $n \geq 1$ ) qui soit de petite hauteur et dont les dérivées sur un ensemble fini de multiples épaissis du point $p$ soient également petites. À cette fin nous avons recours au lemme de Siegel approché absolu élaboré dans [Ga3] (proposition 3.10). Puis, par le lemme d'interpolation analytique du paragraphe précédent (proposition 2.1), nous montrons que le premier jet non nul de cette section en un certain multiple de $p$ est lui-même de petites normes relativement aux plongements du corps de nombres ambiant au-dessus de $\sigma_{0}$ (propositions 3.14 et 3.15). Nous majorons ensuite les normes de ce jet en les autres places, ce 
qui permet d'en déduire une borne pour la hauteur du jet (propositions 3.16 et 3.17) que nous comparons alors à la minoration de type Liouville de la hauteur (proposition 3.21). Nous obtenons ainsi une inégalité assez compliquée où s'entremêlent plusieurs paramètres. Nous divisons alors par l'un des paramètres que nous faisons tendre vers l'infini, ce qui élimine au passage quelques termes secondaires. Nous parvenons à une minoration intermédiaire de $D \widehat{h}_{L}(p)+\frac{\pi}{2} E^{2}\|u\|_{L, \sigma_{0}}^{2}$ (lemmes 3.24 et 3.26) de laquelle découle celle du théorème 3.1 par un bon choix de paramètres. Hormis les lemmes de Siegel et d'interpolation qui diffèrent, ces caractéristiques de la démonstration sont celles de la preuve du théorème des périodes (cas $p=0$ ) obtenu dans [GR2]. La différence est que, comme dans $[\mathrm{PW}$, nous sommes amenés à distinguer deux cas, dits cas périodique et cas non périodique, selon qu'un certain multiple de $p$ appartient ou non à une certaine sous-variété abélienne de $A$ (définition 3.2). Le cas de [GR2] étant nativement le cas périodique, cette distinction n'avait pas lieu d'être. Ici la présence des deux cas nécessite d'utiliser des arguments différents à certains endroits de la démonstration. Ceci est particulièrement visible lors de l'étude des rangs des matrices auxiliaires qui prélude au lemme de Siegel (lemme 3.6) et lors de l'extrapolation analytique proprement dite $(\S 3.9 .1$ et 3.9 .2$)$. Il en résulte une analyse finale qui se dédouble $(\S 3.11 .4$ et 3.11.5). À cette complication inhérente à notre approche s'ajoute la disjonction entre les cas $g=1$ et $g \geq 2$, qui, elle, ne vise qu'à proposer une constante $c_{0}(g)$ plus petite.

Le reste de cette partie est consacré à la démonstration du théorème 3.1.

3.1. Choix des métriques. Soient $(A, L)$ la variété abélienne polarisée de dimension $g$ et $p \in A(k)$ les données de l'énoncé clef. Soient $n$ et $S$ deux entiers $\geq 1$, qui seront définis au paragraphe 3.2. Pour $a \in \mathbb{N}$, posons $\Sigma_{p}(a)=\{m p ; m \in\{-a, \ldots, a\}\}$. Considérons un modèle de Moret-Bailly du triplet $\left(A, L^{\otimes n}, \Sigma_{p}(g S)\right.$ ) (noter que dans [GR2] l'entier $n$ aurait dû être introduit comme ici avant le choix du modèle). La définition, l'existence et les propriétés d'un tel modèle sont l'objet du paragraphe 4.3 de [Bo2]. Rappelons qu'un tel modèle comprend :

- une extension finie $K / k$

- un schéma en groupes $\pi: \mathcal{A} \rightarrow \operatorname{Spec} \mathcal{O}_{K}$ semi-stable, de fibre générique isomorphe à $A_{K}=$ $A \times$ Spec $k \operatorname{Spec} K$

- un fibré hermitien cubiste $\overline{\mathcal{L}_{n}}$ sur $\mathcal{A}$, de fibre générique isomorphe à $L_{K}^{\otimes n}$

- pour tout point $q \in \Sigma_{p}(g S)$, une section $\varepsilon_{q}: \operatorname{Spec} \mathcal{O}_{K} \rightarrow \mathcal{A}$ de $\pi$ qui relève $q$.

Parmi les propriétés décrites dans le théorème 4.10 de [Bo2], mentionnons les deux suivantes :

1) Pour tout $q \in \Sigma_{p}(g S)$, le degré d'Arakelov normalisé du fibré adélique hermitien $\varepsilon_{q}^{*} \overline{\mathcal{L}_{n}}$ est la hauteur de Néron-Tate $\widehat{h}_{L \otimes n}(q)=n \widehat{h}_{L}(q)$ de $q$ relative à $L^{\otimes n}$.

2) Le $K$-espace vectoriel $H_{n}=H^{0}\left(A, L^{\otimes n}\right) \otimes_{k} K$ des sections globales de $L_{K}^{\otimes n}$ est muni d'une structure d'espace adélique hermitien en utilisant le $\mathcal{O}_{K}$-module de type fini (localement libre) $H^{0}\left(\mathcal{A}, \mathcal{L}_{n}\right)$ pour définir les normes aux places finies et les métriques cubistes de $\overline{\mathcal{L}_{n}}$ aux places archimédiennes (voir [GR2, § 6.1]). La pente d'Arakelov normalisée du fibré adélique hermitien $\overline{H_{n}}$ ainsi obtenu vaut

$$
\widehat{\mu}\left(\overline{H_{n}}\right)=-\frac{h(A)}{2}+\frac{1}{4} \log \frac{n^{g} h^{0}(A, L)}{(2 \pi)^{g}}
$$

(où $h^{0}(A, L)$ est la dimension de $H^{0}(A, L)$ ).

Par ailleurs l'espace tangent à l'origine $t_{A_{K}}$ de la variété abélienne $A_{K}$ est également muni d'une structure de fibré adélique hermitien $\overline{t_{A_{K}}}=\left(t_{A_{K}},\left(\|\cdot\|_{L, \sigma}\right)_{\sigma: K \hookrightarrow \mathbb{C}}\right)$ en utilisant l'espace tangent à l'origine $t_{\mathcal{A}}$ de $\mathcal{A}$ comme modèle entier (définissant les métriques aux places ultramétriques) et les formes de Riemann $\|\cdot\|_{L, \sigma}$ des faisceaux inversibles $\left(L_{K}\right)_{\sigma} \rightarrow\left(A_{K}\right)_{\sigma}$ (voir [GR2, § 2.4]). La pente d'Arakelov normalisée de $\overline{t_{A_{K}}}$ est alors égale à

$$
\widehat{\mu}\left(\overline{t_{A_{K}}}\right)=-\frac{1}{g} \cdot\left(h(A)+\frac{1}{2} \log h^{0}(A, L)-\frac{g}{2} \log \pi\right)
$$


(voir [Ga1, Proposition 4.7]). Dans la suite, étant donné un plongement complexe $\sigma: K \hookrightarrow \mathbb{C}$, nous noterons $A_{\sigma}$ (resp. $\left.L_{\sigma}\right)$ la variété abélienne $\left(A_{K}\right)_{\sigma}$ (resp. le faisceau inversible $\left.\left(L_{K}\right)_{\sigma}\right)$. Pour un tel plongement $\sigma$, le tiré en arrière du faisceau inversible hermitien $\overline{\left(\mathcal{L}_{n}\right)_{\sigma}}$ muni de la métrique cubiste $\|\cdot\|_{\text {cub }, \sigma}$ par l'application exponentielle $\exp _{A_{\sigma}}$ est isomorphe (isométriquement) au faisceau trivial $t_{A_{\sigma}} \times \mathbb{C} \rightarrow t_{A_{\sigma}}$ muni en chaque fibre $z$ de la norme caractérisée par $\|1\|_{z, \sigma}=\exp \left(-\pi n\|z\|_{L, \sigma}^{2} / 2\right)$. En prenant les sections globales de ces faisceaux, nous pouvons alors identifier $H^{0}\left(\mathcal{A}, \mathcal{L}_{n}\right) \otimes_{\sigma} \mathbb{C}$ à l'ensemble des fonctions holomorphes $\vartheta: t_{A_{\sigma}} \rightarrow \mathbb{C}$ vérifiant $\vartheta(z+\omega)=a_{L_{\sigma}}(\omega, z)^{n} \vartheta(z)$ pour tout $z \in t_{A_{\sigma}}$ et toute période $\omega$ de $A_{\sigma}$, où $a_{L_{\sigma}}(\omega, z)$ est le facteur d'automorphie canonique de $L_{\sigma}$ (voir par exemple [GR2, § 2.5]). Pour $s \in H^{0}\left(\mathcal{A}, \mathcal{L}_{n}\right) \otimes_{\sigma} \mathbb{C}$ de fonction $\vartheta$ associée et pour $x \in A_{\sigma}(\mathbb{C})$, la norme cubiste de $s(x)$ relative à $\left(\overline{\mathcal{L}_{n}}, \sigma\right)$ est reliée à la forme de Riemann d'un logarithme $z$ quelconque de $x$ et au module de $\vartheta(z)$ par la formule

$$
\|s(x)\|_{\mathrm{cub}, \sigma}=|\vartheta(z)| \exp \left(-\frac{\pi n}{2}\|z\|_{L, \sigma}^{2}\right) .
$$

Plus généralement, si $\mathcal{J}_{\sigma}$ est le faisceau d'idéaux sur $A_{\sigma}$ défini par la section neutre $\varepsilon_{\sigma}:$ Spec $\mathbb{C} \rightarrow$ $A_{\sigma}$, si $s \in H^{0}\left(A_{\sigma}, J_{\sigma}^{\ell} \otimes \tau_{x}^{*}\left(\mathcal{L}_{n}\right)_{\sigma}\right)$ où $\ell \in \mathbb{N}, x \in A_{\sigma}(\mathbb{C})$ et $\tau_{x}: A_{\sigma} \rightarrow A_{\sigma}$ la translation, le jet de $s$ d'ordre $\ell$ au point $x$, noté jet ${ }^{\ell} s(x)$, est l'image de $s$ dans le quotient

$$
H^{0}\left(\operatorname{Spec} \mathbb{C}, \varepsilon_{\sigma}^{*}\left(\mathcal{I}_{\sigma}^{\ell} / \mathcal{I}_{\sigma}^{\ell+1} \otimes_{\mathcal{O}_{A_{\sigma}}} \tau_{x}^{*}\left(\mathcal{L}_{n}\right)_{\sigma}\right)\right) \simeq S^{\ell}\left(t_{A_{\sigma}}^{v}\right) \otimes x^{*}\left(\mathcal{L}_{n}\right)_{\sigma}
$$

$\left(S^{\ell}\left(t_{A_{\sigma}}^{\vee}\right)\right.$ désigne la puissance symétrique $\ell$-ème du cotangent). Cette construction est compatible à la trivialisation de $\exp _{A_{\sigma}}^{*}\left(\mathcal{L}_{n}\right)_{\sigma}$ choisie pour identifier $s$ à $\vartheta$. Ainsi l'on peut définir de la même manière le jet jet ${ }^{\ell} \vartheta(z)$ en un point $z \in t_{A_{\sigma}}$ en considérant $\exp _{A_{\sigma}}^{*} J_{\sigma}^{\ell} \otimes \tau_{z}^{*}\left(\exp _{A_{\sigma}}^{*}\left(\mathcal{L}_{n}\right)_{\sigma}\right) \operatorname{sur}_{A_{\sigma}}$ et ce vecteur appartient à $S^{\ell}\left(t_{A_{\sigma}}^{\vee}\right)$. Les normes de ces jets sont alors reliées par la formule (pour $\left.x=\exp _{A_{\sigma}}(z)\right)$

$$
\left\|\operatorname{jet}^{\ell} s(x)\right\|_{S^{\ell}\left(t_{A_{\sigma}}^{v}\right) \otimes x^{*}\left(\mathcal{L}_{n}\right)_{\sigma}}=\left\|\operatorname{jet}^{\ell} \vartheta(z)\right\|_{S^{\ell}\left(t_{A_{\sigma}}^{\mathrm{v}}\right.}\|1\|_{z, \sigma}=\left\|\operatorname{jet}^{\ell} \vartheta(z)\right\|_{S^{\ell}\left(t_{A_{\sigma}}^{\mathrm{v}}\right)} \exp \left(-\frac{\pi n}{2}\|z\|_{L, \sigma}^{2}\right) .
$$

Au paragraphe 3.8 nous verrons une formule pour la norme $\operatorname{de~jet~}^{\ell} \vartheta(z)$ en fonction des dérivées de $\vartheta$ à l'ordre $\ell$ au point $z$.

Remarque. Nous aurons à considérer les plongements de $K$ qui prolongent le plongement $\sigma_{0}: k \hookrightarrow$ $\mathbb{C}$ de l'énoncé du théorème 3.1 ainsi que ceux qui prolongent le conjugué complexe $\overline{\sigma_{0}}$ (lorsque $\sigma_{0}$ n'est pas un plongement réel). Or le morphisme naturel de $\mathbb{R}$-schémas $\mathrm{f}: A_{\overline{\sigma_{0}}} \rightarrow A_{\sigma_{0}}$ se relève en un isomorphisme antilinéaire et isométrique $\mathrm{df}:\left(t_{A_{\overline{\sigma_{0}}}},\|\cdot\|_{L, \overline{\sigma_{0}}}\right) \rightarrow\left(t_{A_{\sigma_{0}}},\|\cdot\|_{L, \sigma_{0}}\right)$ des espaces tangents normés, avec correspondance des réseaux des périodes (voir [GR2, Proposition 2.2]). Dans la suite les constructions faites pour les plongements $\sigma \mid \sigma_{0}$ seront systématiquement transposées aux plongements au-dessus de $\overline{\sigma_{0}}$ via $\mathrm{f}$ (ou df), assurant ainsi des bornes identiques pour les normes associées à $\sigma$ ou $\bar{\sigma}$. En particulier le logarithme choisi pour $\overline{\sigma_{0}}(p)$ sera $\bar{u} \in t_{A_{\overline{\sigma_{0}}}}$ tel que $\operatorname{df}(\bar{u})=u$, ce qui implique $\|\bar{u}\|_{L, \overline{\sigma_{0}}}=\|u\|_{L, \sigma_{0}}$.

3.2. Choix des paramètres. À partir de maintenant et jusqu'à la fin du $\S 3.12$, nous fixons $k$, $\sigma_{0}, A, L, u, p$ et $E$ comme dans l'énoncé du théorème 3.1. Soit

$$
M:=\max \left\{1, \frac{D}{\log E}, \frac{D}{\log E} \log \frac{D}{\log E}, \frac{D h(A)}{\log E}, \frac{D \log \operatorname{deg}_{L} A}{\log E}\right\} .
$$

Soit $\widetilde{S}$ le nombre réel défini par $\widetilde{S}:=2919 M$ si $g=1$, et lorsque $g \geq 2$, par $\widetilde{S}:=248 g^{g+7}(\log g) M$. Notons alors $S:=[\widetilde{S}]$. Lorsque $B$ est une sous-variété abélienne stricte de $A_{\sigma_{0}}$, posons

$$
x(B):=\left(\operatorname{Card}\left(\frac{\Sigma_{p}(S)+B}{B}\right) \frac{\operatorname{deg}_{L} B}{\operatorname{deg}_{L} A}\right)^{1 / \operatorname{codim} B} \quad \text { et } \quad x:=\min \left\{x(B) \mid B \subsetneq A_{\sigma_{0}}\right\}
$$


(rappelons que $\left.\Sigma_{p}(S)=\{m p ; m \in\{-S, \ldots, S\}\}\right)$. Choisissons une sous-variété abélienne stricte $B_{0}$ de $A_{\sigma_{0}}$ telle que $x\left(B_{0}\right)=x$. Nous disposons de l'encadrement

$$
\frac{1}{\operatorname{deg}_{L} A} \leq x \leq x(\{0\}) \leq\left(\frac{2 S+1}{\operatorname{deg}_{L} A}\right)^{1 / g} .
$$

Notons $\Omega_{A_{\sigma_{0}}}$ le réseau des périodes de $A_{\sigma_{0}}$ et $t_{B_{0}}$ l'espace tangent à l'origine de la sous-variété abélienne $B_{0}$.

Définition 3.2. Nous dirons que nous sommes dans le cas non périodique si le cardinal du quotient $\left(\Sigma_{p}(S)+B_{0}\right) / B_{0}$ est maximal, c'est-à-dire égal à $2 S+1$. Sinon nous dirons que nous sommes dans le cas périodique.

En termes de logarithmes, être dans le cas périodique signifie qu'il existe $m \in\{1,2, \ldots, 2 S\}$ tel que $m u \in t_{B_{0}}+\Omega_{A_{\sigma_{0}}}$. On introduit alors le nombre réel $\varepsilon_{0}>0$ de la manière suivante. Dans le cas non périodique, on pose

$$
\varepsilon_{0}=0,044 \quad \text { si } g=1 \quad \text { et } \quad \varepsilon_{0}=\frac{0,57}{4 g \log (g+1)+4 \log g+7,31} \quad \text { si } g \geq 2 .
$$

Dans le cas périodique, on pose

$$
\varepsilon_{0}=0,06 \quad \text { si } g=1 \quad \text { et } \quad \varepsilon_{0}=\frac{3}{50 g^{g}+3} \quad \text { si } g \geq 2 .
$$

Considérons maintenant un entier $T \geq 1$ et $n$ l'entier tel que $n<T x \leq n+1$. Le paramètre $T$ tendra vers $+\infty$ en fin de démonstration, nous pouvons donc supposer que $n \geq 2$ dans la suite, ce que nous ferons. Nous notons $K / k$ une extension finie pour laquelle nous disposons d'un modèle de Moret-Bailly de $\left(A, L^{\otimes n}, \Sigma_{p}(g S)\right)$ (paragraphe précédent).

Avec ces choix de paramètres, nous avons le lemme de multiplicité suivant, une clôture algébrique $\bar{K}$ de $K$ étant fixée.

Proposition 3.3. Il n'existe aucune section non nulle de $H_{n} \otimes_{K} \bar{K}$ qui s'annule à l'ordre gT le long de $t_{A}$ sur $\Sigma_{p}(g S)$.

Démonstration. Si une telle section existait, le lemme de multiplicité de Nakamaye [Na] assurerait l'existence d'une sous-variété abélienne stricte $B$ de $A_{\sigma_{0}}$ telle que

$$
T^{\operatorname{codim} B} \operatorname{Card}\left(\frac{\Sigma_{p}(S)+B}{B}\right) \operatorname{deg}_{L \otimes n} B \leq \operatorname{deg}_{L^{\otimes n}} A=n^{g} \operatorname{deg}_{L} A .
$$

On aurait donc $T x \leq T x(B) \leq n$, ce qui est impossible puisque $T x>n$ par définition de $n$.

Nous utiliserons aussi trois autres paramètres $\widetilde{S}_{0}, S_{0}$ et $T_{0}$ définis de la manière suivante. Le nombre réel $\widetilde{S}_{0}$ est déterminé par la formule

$$
2 \widetilde{S}_{0}+1=\varepsilon_{0}(g+1)^{-g}(2 S+1)
$$

puis l'on pose $S_{0}:=\left[\widetilde{S}_{0}\right]$. Nous notons également $T_{0}:=\left[\varepsilon_{0} T\right]$. Les nombres $\widetilde{S}_{0}$ et $S_{0}$ ne seront utilisés que dans le cas non périodique et $T_{0}$ uniquement dans le cas périodique. Leur utilisation ne peut se faire que dans le cas associé. Afin de pouvoir traiter les cas simultanément, nous noterons

$$
\left(S_{1}, T_{1}\right)= \begin{cases}\left(S_{0}, T\right) & \text { dans le cas non périodique } \\ \left(g S, T_{0}\right) & \text { dans le cas périodique. }\end{cases}
$$

Nous aurons besoin dans la suite de savoir que le paramètre $S_{0}$ est grand :

Lemme 3.4. En notant $\kappa(1)=62$ et $\kappa(g)=4 g^{6}$ si $g \geq 2$, on a la minoration :

$$
S_{0} \geq \kappa(g) \max \left\{1, \frac{D}{\log E}, \frac{D}{\log E} \log \frac{D}{\log E}, \frac{D h(A)}{\log E}, \frac{D \log \operatorname{deg}_{L} A}{\log E}\right\} .
$$


Démonstration. Supposons d'abord $g \geq 2$. D'après les définitions de $\widetilde{S}_{0}$ et $\widetilde{S}$, on a :

$$
2 \widetilde{S}_{0}+1=\frac{\varepsilon_{0}(2 S+1)}{(g+1)^{g}} \geq \frac{\varepsilon_{0}(2 \widetilde{S}-1)}{(g+1)^{g}} \geq \frac{495 \varepsilon_{0}}{(1+1 / g)^{g}} \times g^{7}(\log g) M .
$$

Cette dernière quantité est supérieure à $9,2 g^{6} M$. Comme $\widetilde{S}_{0} \leq S_{0}+1$, on en déduit $2 S_{0} \geq 2 \widetilde{S}_{0}-2 \geq$ $9 g^{6} M$, d'où le lemme dans le cas $g \geq 2$. Le cas $g=1$ se démontre de façon analogue : on trouve d'abord

$$
2 \widetilde{S}_{0}+1=\frac{\varepsilon_{0}}{2}(2 S+1) \geq \frac{0,044 \times(2 \times 2919-1)}{2} M \geq 128 M,
$$

d'où $2 \widetilde{S}_{0} \geq 127 M$ puis $S_{0} \geq \widetilde{S}_{0}-1 \geq 62 M$.

Pour $\tau=\left(\tau_{1}, \ldots, \tau_{g}\right) \in \mathbb{N}^{g}$, notons $|\tau|$ la somme $\tau_{1}+\cdots+\tau_{g}$. Définissons alors les ensembles

$$
\Upsilon:=\left\{(m, \tau) \in \mathbb{Z} \times \mathbb{N}^{g} ;|m| \leq S_{0} \text { et }|\tau| \leq(g+1) T\right\}
$$

dans le cas non périodique, et

$$
\Upsilon:=\left\{(m, \tau) \in \mathbb{Z} \times \mathbb{N}^{g} ;|m| \leq g S \text { et }|\tau| \leq g T+T_{0}, \tau_{g} \leq T_{0}\right\}
$$

dans le cas périodique. Le cardinal de $\Upsilon$ est un polynôme en $T$ et, en particulier, $\log \operatorname{Card} \Upsilon=o(T)$ est négligeable devant $T$ lorsque $T$ tend vers l'infini.

3.3. Compléments au cas périodique. Notons dist la distance sur $t_{A_{\sigma_{0}}}$ induite par la forme de Riemann $\|\cdot\|_{L, \sigma_{0}}$. Considérons une base orthonormée $\mathbf{f}=\left(f_{1}, \ldots, f_{g}\right)$ de $t_{A_{\sigma_{0}}}$ telle que

$$
f_{g}= \begin{cases}u /\|u\|_{L, \sigma_{0}} & \text { dans le cas non périodique, } \\ v /\|v\|_{L, \sigma_{0}} & \text { dans le cas périodique }\end{cases}
$$

où $v$ est le projeté orthogonal de $u$ parallèlement à $t_{B_{0}}$ (non nul puisque $u \notin t_{B_{0}}$ ). Sur $t_{A_{\overline{\sigma_{0}}}}$, nous considérons la base orthonormée $\overline{\mathbf{f}}$ compatible à $\mathbf{f}$ au sens de la remarque du paragraphe 3.1.

Dans le cas périodique, nous aurons besoin d'une minoration de la dernière coordonnée de $u$ dans la base $\mathbf{f}$ :

Proposition 3.5. Notons $\left(u_{1}, \ldots, u_{g}\right)$ les coordonnées de u dans la base $\mathbf{f}$. Dans le cas périodique, on a $u_{g}>0$, ainsi que les estimations :

(i) Si $g=1$,

$$
\frac{1}{u_{g}} \leq 2 S \sqrt{6,45 D}\left(\operatorname{deg}_{L} A\right)^{-1 / 2} \max \{1, h(A)\}^{1 / 2} .
$$

(ii) Si $g \geq 2$,

$$
\left(\frac{\left(\operatorname{deg}_{L} A\right)^{1 / g}}{D u_{g}^{2}}\right)^{1 / 2} \leq 2 S(2 S+1)^{g} \sqrt{131} g^{g+3} \max \left\{h(A), \log \operatorname{deg}_{L} A, \log (2 S+1)\right\}^{1 / 2} .
$$

Démonstration. Par définition du cas périodique, il existe un entier $m \in\{1,2, \ldots, 2 S\}$ tel que $m u \in t_{B_{0}}+\Omega_{A_{\sigma_{0}}}$. Soit $\omega \in \Omega_{A_{\sigma_{0}}}$ une période telle que $\operatorname{dist}\left(m u, t_{B_{0}}\right)=\operatorname{dist}\left(\omega, t_{B_{0}}\right)$. Compte tenu du choix de la base $\mathbf{f}$, on a $u_{g}=\|v\|_{L, \sigma_{0}}$ donc

$$
\operatorname{dist}\left(\omega, t_{B_{0}}\right)=\operatorname{dist}\left(m u, t_{B_{0}}\right)=m \operatorname{dist}\left(u, t_{B_{0}}\right)=m u_{g} .
$$

Nous en déduisons

$$
2 S u_{g} \geq \operatorname{dist}\left(\omega, t_{B_{0}}\right) \geq \min \left\{\operatorname{dist}\left(\omega^{\prime}, t_{B_{0}}\right) ; \omega^{\prime} \in \Omega_{A_{\sigma_{0}}} \backslash \Omega_{B_{0}}\right\}=: \delta .
$$

Il suffit donc de minorer $\delta$. Si $g=1$ alors $\delta$ est égal au diamètre d'injectivité $\rho\left(A_{\sigma_{0}}, L_{\sigma_{0}}\right):=$ $\min \left\{\left\|\omega^{\prime}\right\|_{L, \sigma_{0}} ; \omega^{\prime} \in \Omega_{A_{\sigma_{0}}} \backslash\{0\}\right\}$ de $\left(A_{\sigma_{0}}, L_{\sigma_{0}}\right)$. En notant $L_{0}$ l'unique polarisation principale de la courbe elliptique $A$, nous avons alors $L=L_{0}^{\otimes \operatorname{deg}_{L} A}$ et $\rho\left(A_{\sigma_{0}}, L_{\sigma_{0}}\right)^{2}=\left(\operatorname{deg}_{L} A\right) \rho\left(A_{\sigma_{0}},\left(L_{0}\right)_{\sigma_{0}}\right)^{2}$. La partie (i) de la proposition découle alors de la proposition 3.2 de [GR2] (conséquence du lemme 
matriciel d'Autissier $[\mathrm{Au}]): \rho\left(A_{\sigma_{0}},\left(L_{0}\right)_{\sigma_{0}}\right)^{-2} \leq 6,45 D \max \{1, h(A)\}$. Supposons maintenant $g \geq 2$. Si $B$ est une sous-variété abélienne stricte de $A_{\sigma_{0}}$, posons

$$
y(B)=\left(\frac{\operatorname{deg}_{L} B}{\operatorname{deg}_{L} A}\right)^{1 / \operatorname{codim} B} \quad \text { et } \quad y:=\min \left\{y(B) ; B \subsetneq A_{\sigma_{0}}\right\} .
$$

Observons maintenant que, pour toute sous-variété abélienne stricte $B$ de $A_{\sigma_{0}}$, on a $y(B) \leq x(B) \leq$ $(2 S+1) y(B)$, ce qui entraîne immédiatement $y\left(B_{0}\right) \leq x \leq(2 S+1) y$. En utilisant la majoration $x \leq\left((2 S+1) / \operatorname{deg}_{L} A\right)^{1 / g}$ nous avons donc

$$
\operatorname{deg}_{L} B_{0}=y\left(B_{0}\right)^{\operatorname{codim} B_{0}} \operatorname{deg}_{L} A \leq \max \left\{\operatorname{deg}_{L} A, 2 S+1\right\} .
$$

Cette même majoration de $x$ fournit également

$$
\begin{aligned}
y\left(\frac{y\left(B_{0}\right)}{y}\right)^{2 \operatorname{codim} B_{0}} & =y\left(B_{0}\right)\left(\frac{y\left(B_{0}\right)}{y}\right)^{2 \operatorname{codim} B_{0}-1} \\
& \leq x\left(\frac{x}{y}\right)^{2 \operatorname{codim} B_{0}-1} \leq x(2 S+1)^{2 g-1} \leq(2 S+1)^{2 g}\left(\operatorname{deg}_{L} A\right)^{-1 / g} .
\end{aligned}
$$

Appliquons alors le théorème des périodes 4.5 de [GR2] à l'image $B[\sigma]$ de $B_{0}$ par un isomorphisme $A_{\sigma_{0}} \rightarrow A_{\sigma}$ pour chaque plongement $\sigma: k \hookrightarrow \mathbb{C}:$

$$
\frac{1}{\delta^{2}} \leq 131 D g^{2 g+6} y\left(\frac{y\left(B_{0}\right)}{y}\right)^{2 \operatorname{codim} B_{0}} \max \left\{1, h(A), \log \operatorname{deg}_{L} A, \log \operatorname{deg}_{L} B_{0}\right\} .
$$

En réunissant les estimations précédentes, nous obtenons la majoration (ii) de la proposition.

3.4. Fibré adélique hermitien des sections auxiliaires. Nous noterons dans la suite

$$
\nu=\operatorname{dim} H^{0}\left(A, L^{\otimes n}\right)=n^{g} h^{0}(A, L) .
$$

Pour $\sigma: K \hookrightarrow \mathbb{C}$ qui prolonge $\sigma_{0}$, considérons une base orthonormée $\left(s_{1}, \ldots, s_{\nu}\right)$ de l'espace hermitien $\left(H_{n} \otimes_{\sigma} \mathbb{C},\|\cdot\|_{\overline{H_{n}}, \sigma}\right)$ introduit au paragraphe 3.1 et $\vartheta_{1}, \ldots, \vartheta_{\nu}$ les fonctions thêta associées aux éléments de cette base. Soit $U_{\sigma} \in M_{\mathrm{Card} \Upsilon, \nu}(\mathbb{C})$ la matrice dont les coefficients sont donnés par

$$
U_{\sigma}[(m, \tau), i]=\frac{1}{\tau !} D_{\mathbf{f}}^{\tau} \vartheta_{i}(m u) \exp \left(-\frac{\pi}{2} n\|m u\|_{L, \sigma_{0}}^{2}\right)
$$

pour $(m, \tau) \in \Upsilon$ et $1 \leq i \leq \nu$. Dans cette expression $\mathbf{f}$ est la base de $t_{A_{\sigma_{0}}}$ introduite au début du paragraphe précédent et $\frac{1}{\tau !} D_{\mathbf{f}}^{\tau} \vartheta_{i}(m u)$ est la dérivée divisée de $\vartheta_{i}$ le long de la base $\mathbf{f}$ de $t_{A_{\sigma_{0}}}$ à l'ordre $\tau=\left(\tau_{1}, \ldots, \tau_{g}\right)$ au point $m u$, c'est-à-dire le coefficient devant $z^{\tau}=z_{1}^{\tau_{1}} \cdots z_{g}^{\tau_{g}}$ de la série de Taylor de $\vartheta_{i}\left(m u+z_{1} f_{1}+\cdots+z_{g} f_{g}\right)$. Nous définissons de la même manière une matrice $U_{\bar{\sigma}}$ en utilisant la base $\overline{\mathbf{f}}$ de $t_{A_{\overline{\sigma_{0}}}}$ compatible à $\mathbf{f}$.

Fixons un nombre réel $\epsilon$ tel que $0<\epsilon<1 / 2$ (qui tendra vers 0 par la suite) et définissons les nombres réels $P$ et $Q_{\epsilon}$ de la façon suivante. Dans le cas non périodique, posons

$$
\begin{aligned}
P=( & \left.\frac{1}{E}\right)^{\left(2 S_{0}+1\right)(T+1)}\left(1+\frac{\varepsilon_{0}^{2}}{2,9 g^{2}(g+1)^{2 g}}\right)^{S_{0}(T+1)} \exp \left(T+\frac{\pi}{2} n(g S)^{2} E^{2}\|u\|_{L, \sigma_{0}}^{2}\right) \\
& \times \max \left\{1, \pi x \sqrt{g}\left(E g S\|u\|_{L, \sigma_{0}}+\sqrt{1 /(2 \pi x)}\right)\right\}^{g T}
\end{aligned}
$$

et

$$
Q_{\epsilon}=2^{g T} \max \left\{1,\|u\|_{L, \sigma_{0}}\right\}^{T}\left(\frac{1}{\cos \pi \epsilon}\right)^{T+1}\left(\frac{g S}{S_{0}}\right)^{\left(2 S_{0}+1\right)(T+1)}\left(\operatorname{sh}\left(\pi S_{0}\right)\right)^{T+1}
$$


Dans le cas périodique, posons

$$
\begin{gathered}
P=\left(\frac{4}{3}\right)^{g S\left(T_{0}+1\right)}\left(\frac{1}{E}\right)^{(2 g S+1)\left(T_{0}+1\right)} \exp \left(T+\frac{\pi}{2} n E^{2}(g S+1)^{2}\|u\|_{L, \sigma_{0}}^{2}\right) \\
\times \max \left\{1, \pi x \sqrt{g}\left(E(g S+1)\|u\|_{L, \sigma_{0}}+\sqrt{1 /(2 \pi x)}\right)\right\}^{g T}
\end{gathered}
$$

et

$$
Q_{\epsilon}=2^{g T} \max \left\{1,\|u\|_{L, \sigma_{0}}\right\}^{T_{0}}\left(\frac{1}{\cos \pi \epsilon}\right)^{T_{0}+1}(\operatorname{sh}(\pi(g S+1)))^{T_{0}+1} \exp \left(\frac{\pi}{2} n(g S)^{2}\|u\|_{L, \sigma_{0}}^{2}\right) .
$$

Définissons alors le nombre réel $\alpha>0$ par $\alpha:=Q_{\epsilon} / P$. Pour $\left(x_{1}, \ldots, x_{\nu}\right) \in \mathbb{C}^{\nu}$ et $\sigma \mid \sigma_{0}$, posons

$$
\left\|x_{1} s_{1}+\cdots+x_{\nu} s_{\nu}\right\|_{H_{n, \alpha}, \sigma}^{2}:=\left|x_{1}\right|^{2}+\cdots+\left|x_{\nu}\right|^{2}+\left|\alpha U_{\sigma}\left(\begin{array}{c}
x_{1} \\
\vdots \\
x_{\nu}
\end{array}\right)\right|_{2}^{2} .
$$

Ceci confère à $H_{n} \otimes_{\sigma} \mathbb{C}$ une norme hermitienne, qui se transporte par conjugaison complexe sur $H_{n} \otimes_{\bar{\sigma}} \mathbb{C}$ (norme définie par la matrice $U_{\bar{\sigma}}$ ). En les plongements (complexes ou $p$-adiques) $\sigma$ de $K$ différents de ceux au-dessus de $\sigma_{0}$ ou $\overline{\sigma_{0}}$, nous munissons $H_{n} \otimes_{\sigma} \mathbb{C}_{p}$ de la norme donnée par $\overline{H_{n}}$. Nous obtenons ainsi un fibré adélique hermitien $\bar{H}_{n, \alpha}$ sur $K$

$$
\bar{H}_{n, \alpha}=\left(H^{0}\left(A, L^{\otimes n}\right) \otimes_{k} K,\left(\|\cdot\|_{\bar{H}_{n, \alpha}, \sigma}\right)_{\sigma}\right) .
$$

3.5. Estimations des rangs des matrices auxiliaires. Soit $\sigma: K \hookrightarrow \mathbb{C}$ un plongement qui prolonge $\sigma_{0}$ ou $\overline{\sigma_{0}}$.

Lemme 3.6. Dans le cas non périodique, le rang $\rho_{\sigma}$ de $U_{\sigma}$ est majoré par

$$
\left(\begin{array}{c}
(g+1) T+\operatorname{codim} B_{0} \\
\operatorname{codim} B_{0}
\end{array}\right) \operatorname{Card}\left(\frac{\Sigma_{p}\left(S_{0}\right)+B_{0}}{B_{0}}\right) \frac{\operatorname{deg}_{L} B_{0}}{\left(\operatorname{dim} B_{0}\right) !} n^{\operatorname{dim} B_{0}}
$$

et, dans le cas périodique, par

$$
X \text { Card }\left(\frac{\Sigma_{p}(S)+B_{0}}{B_{0}}\right) \frac{\operatorname{deg}_{L} B_{0}}{\left(\operatorname{dim} B_{0}\right) !} n^{\operatorname{dim} B_{0}}
$$

où

$$
X=\left(\begin{array}{c}
g T+T_{0}+\operatorname{codim} B_{0} \\
\operatorname{codim} B_{0}
\end{array}\right)-\left(\begin{array}{c}
g T-1+\operatorname{codim} B_{0} \\
\operatorname{codim} B_{0}
\end{array}\right)
$$

Démonstration. Comme $\rho_{\sigma}=\rho_{\bar{\sigma}}$, nous pouvons supposer que $\sigma$ prolonge $\sigma_{0}$. Comme nous l'avons rappelé page 8 , nous pouvons identifier l'espace complexe $F_{-1}=\left(H^{0}\left(A, L^{\otimes n}\right) \otimes_{k} K\right) \otimes_{\sigma} \mathbb{C}$ à l'ensemble des fonctions holomorphes $\vartheta: t_{A_{\sigma_{0}}} \rightarrow \mathbb{C}$ vérifiant $\vartheta(z+\omega)=a_{L}(\omega, z)^{n} \vartheta(z)$ pour tous $z \in t_{A_{\sigma_{0}}}$ et $\omega \in \Omega_{A_{\sigma_{0}}}$, où $a_{L}(\omega, z)$ est le facteur d'automorphie canonique de $L_{\sigma_{0}}$. Considérons une base $\mathfrak{f}$ de $t_{A_{\sigma_{0}}}$ dont les $\operatorname{dim} B_{0}$ premiers vecteurs forment une base de $t_{B_{0}}$ et telle que, dans le cas périodique, le dernier vecteur de cette base soit proportionnel au projeté orthogonal de $u$ parallèlement à $t_{B_{0}}$. Cette dernière caractéristique assure que l'application $\iota: F_{-1} \rightarrow \mathbb{C}^{\text {Card } \Upsilon}$ qui à $\vartheta$ associe $\left(D_{\mathfrak{f}}^{\tau} \vartheta(m u)\right)_{(m, \tau) \in \Upsilon}$ est de même rang $\rho_{\sigma}$ que $U_{\sigma}$. Pour $\ell \in \mathbb{N}$, considérons le sous-espace $F_{\ell} \subset F_{-1}$ des fonctions $\vartheta$ telles que, pour tout entier $m$ de valeur absolue $\leq S_{1}$ (défini page 9 ) et tout multiplet $\tau=\left(0, \ldots, 0, \tau_{\operatorname{dim} B_{0}+1}, \ldots, \tau_{g}\right) \in \mathbb{N}^{g}$ de longueur $\leq \ell$ avec la condition supplémentaire $\tau_{g} \leq T_{0}$ dans le cas périodique, l'application restreinte $\xi \in t_{B_{0}} \mapsto D_{\mathfrak{f}}^{\tau} \vartheta(m u+\xi)$ soit identiquement nulle. La suite $\left(F_{\ell}\right)_{\ell \in \mathbb{N}}$ est emboîtée et $F_{g T+T_{1}} \subset$ ker $\iota$. Nous affirmons que, dans la définition de $F_{\ell}$, nous pouvons remplacer chaque $m u$ par un translaté de celui-ci par un élément quelconque de $t_{B_{0}}+\Omega_{A_{\sigma_{0}}}$. En effet l'invariance par translation par un élément de $t_{B_{0}}$ découle immédiatement de la définition de $F_{\ell}$. De plus, si $\omega$ est une période de $A_{\sigma_{0}}$ alors la relation avec le facteur d'automorphie rappelée en préambule et le théorème de dérivation de Leibniz montrent que si $\vartheta$ appartient à $F_{\ell}$ il en est de même pour $\vartheta(\cdot+\omega)$. Grâce à cette observation l'on peut remplacer les $m u,|m| \leq S_{1}$, 
qui apparaissent dans la définition de $F_{\ell}$, par une famille complète de représentants $\alpha_{1}, \ldots, \alpha_{h}$ de $\left\{m u ;|m| \leq S_{1}\right\}$ modulo $t_{B_{0}}+\Omega_{A_{\sigma_{0}}}$. Notons d'ailleurs que $h$ est le cardinal du quotient $\left(\Sigma_{p}\left(S_{1}\right)+B_{0}\right) / B_{0}$. En outre, pour $1 \leq i \leq h$ et $\tau=\left(0, \ldots, 0, \tau_{\operatorname{dim} B_{0}+1}, \ldots, \tau_{g}\right) \in \mathbb{N}^{g}$ de longueur $\leq$ $\ell$, avec $\tau_{g} \leq T_{0}$ dans le cas périodique, et pour $\vartheta \in F_{\ell-1}$, l'application $\xi \in t_{B_{0}} \mapsto D_{\mathfrak{f}}^{\tau} \vartheta\left(\alpha_{i}+\xi\right)$ définit une fonction thêta sur $t_{B_{0}}$ puisqu'elle vérifie la relation avec le facteur d'automorphie (restreint à $\left.\Omega_{B_{0}} \times t_{B_{0}}\right)$ qui caractérise les éléments de $H^{0}\left(B_{0}, L_{\sigma}^{\otimes n}\right)$, comme on le voit immédiatement en utilisant à nouveau le théorème de dérivation de Leibniz et l'hypothèse $\vartheta \in F_{\ell-1}$. Si l'on note $X_{\ell}=\operatorname{Card}\left\{\tau^{\prime}=\left(\tau_{\operatorname{dim}_{B_{0}}+1}, \ldots, \tau_{g}\right) \in \mathbb{N}^{g-\operatorname{dim} B_{0}} ;\left|\tau^{\prime}\right|=\ell\right\}$ dans le cas non périodique et $X_{\ell}$ le cardinal de l'ensemble analogue avec la condition supplémentaire $\tau_{g} \leq T_{0}$ dans le cas périodique, on en déduit une injection du quotient $F_{\ell-1} / F_{\ell}$ dans $h X_{\ell}$ copies de $H^{0}\left(B_{0}, L_{\sigma}^{\otimes n}\right)$. En sommant de $\ell=0$ jusqu'à $g T+T_{1}$, nous obtenons une borne pour $\operatorname{dim} F_{-1}-\operatorname{dim} F_{g T+T_{1}}$ puis pour $\rho_{\sigma}$ (car $\left.F_{g T+T_{1}} \subset \operatorname{ker} \iota\right)$ de la forme $\rho_{\sigma} \leq h X \operatorname{dim} H^{0}\left(B_{0}, L_{\sigma}^{\otimes n}\right)$ où $X=\sum_{\ell=0}^{g T+T_{1}} X_{\ell}$ est le cardinal des multiplets de $\mathbb{N}^{g-\operatorname{dim} B_{0}}$ de longueur $\leq g T+T_{1}$ et tels que, dans le cas périodique, la dernière coordonnée soit plus petite que $T_{0}$. Cet entier $X$ est celui du théorème dans le cas périodique et il est égal au coefficient binomial $\left(\begin{array}{c}(g+1) T+\operatorname{codim} B_{0} \\ \operatorname{codim} B_{0}\end{array}\right)$ dans le cas non périodique. Pour conclure, il ne reste plus qu'à observer que la dimension de $H^{0}\left(B_{0}, L_{\sigma}^{\otimes n}\right)$ est $\left(\operatorname{deg}_{L} B_{0}\right) n^{\operatorname{dim} B_{0}} /\left(\operatorname{dim} B_{0}\right)$ ! par amplitude de $L$ et qu'à remplacer $S_{1}$ par sa valeur dans $h$, en notant que, dans le cas périodique, les ensembles $\left(\Sigma_{p}(g S)+B_{0}\right) / B_{0}$ et $\left(\Sigma_{p}(S)+B_{0}\right) / B_{0}$ sont égaux.

Dans la conséquence qui suit, on désigne par $o(1)$ une fonction de $T$ tendant vers 0 lorsque $T$ tend vers l'infini.

Proposition 3.7. Dans le cas non périodique, on a:

$$
\frac{\rho_{\sigma}}{\nu} \leq(g+1)^{g}\left(\frac{2 S_{0}+1}{2 S+1}\right)(1+o(1))
$$

et dans le cas périodique,

$$
\frac{\rho_{\sigma}}{\nu} \leq \eta(1+o(1))
$$

où $\eta=\varepsilon_{0}$ si $g=1$ et $\eta=g^{g} \varepsilon_{0}\left(1-\varepsilon_{0}\right)^{-1}$ si $g \geq 2$.

En réalité le choix de $\varepsilon_{0}$ dans le cas périodique fournit $\eta=0,06$ quel que soit $g$.

Démonstration. Dans le cas non périodique le cardinal de $\left(\Sigma_{p}(S)+B_{0}\right) / B_{0}$ est par définition égal à $2 S+1$. A fortiori celui de $\left(\Sigma_{p}\left(S_{0}\right)+B_{0}\right) / B_{0}$ vaut $2 S_{0}+1$. En utilisant alors la définition de $x\left(B_{0}\right)$ et l'égalité $\nu=n^{g}\left(\operatorname{deg}_{L} A\right) / g$ !, le lemme 3.6 donne

$$
\frac{\rho_{\sigma}}{\nu} \leq \frac{((g+1) T)^{\operatorname{codim} B_{0}}}{\left(\operatorname{codim} B_{0}\right) !} \times \frac{2 S_{0}+1}{2 S+1} \times \frac{x\left(B_{0}\right)^{\operatorname{codim} B_{0}}}{\left(\operatorname{dim} B_{0}\right) !} \times \frac{g !}{n^{\operatorname{codim} B_{0}}}(1+o(1)) .
$$

La relation $n / T=x\left(B_{0}\right)(1+o(1))$ qui vient de la définition de $n=\lceil T x-1\rceil$ entraîne alors

$$
\frac{\rho_{\sigma}}{\nu} \leq(g+1)^{\operatorname{codim} B_{0}}\left(\begin{array}{c}
g \\
\operatorname{dim} B_{0}
\end{array}\right) \frac{2 S_{0}+1}{2 S+1}(1+o(1)) .
$$

Il ne reste plus qu'à majorer le coefficient binomial par $(g+1)^{\operatorname{dim} B_{0}}$ pour obtenir la majoration souhaitée. Dans le cas périodique l'approche est identique ; l'on fait apparaître $x\left(B_{0}\right)$ dans le lemme 3.6 et nous utilisons

$$
\frac{X}{T^{\operatorname{codim} B_{0}}}=\frac{1}{\left(\operatorname{codim} B_{0}\right) !}\left(\left(g+\frac{T_{0}}{T}\right)^{\operatorname{codim} B_{0}}-g^{\operatorname{codim} B_{0}}\right)(1+o(1)) .
$$

En découle la relation

$$
\frac{\rho_{\sigma}}{\nu} \leq g^{\operatorname{codim} B_{0}}\left(\begin{array}{c}
g \\
\operatorname{dim} B_{0}
\end{array}\right)\left(\left(1+\frac{T_{0}}{g T}\right)^{\operatorname{codim} B_{0}}-1\right)(1+o(1)) .
$$


Remplaçons le coefficient binomial par $g^{\operatorname{dim} B_{0}}$ et $T_{0}=\left[\varepsilon_{0} T\right]$ par $\varepsilon_{0} T$. Si $g=1$ nous obtenons alors la majoration souhaitée pour $\rho_{\sigma} / \nu$. Si $g \geq 2$, nous exploitons la décroissance de la fonction

$$
\xi \mapsto(1-\xi)\left(\left(1+\frac{\xi}{g}\right)^{g}-1\right)-\xi
$$

sur l'intervalle $[0,1]$ pour substituer $\varepsilon_{0}\left(1-\varepsilon_{0}\right)^{-1}$ à $\left(1+\varepsilon_{0} / g\right)^{g}-1$ et conclure.

3.6. Construction de la section auxiliaire. Rappelons que $T$ est un paramètre qui tendra vers $+\infty$ en fin de démonstration. Nous appellerons constantes les paramètres ne dépendant pas de $T$, autrement dit $E, \varepsilon_{0}, \widetilde{S}$ (et donc $S, S_{0}$ et $\widetilde{S}_{0}$ ), le nombre réel $\varepsilon$ fixé au $\S 3.4$, et les quantités liées au corps $k$, au point $u$ ou à la variété abélienne polarisée $(A, L)$ (notons que les paramètres $n$ et $T_{0}$ ne sont pas des constantes et tendent vers l'infini avec $T$ ). En particulier, nous utiliserons dans la suite les notations de Landau $o(1), o(T)$, etc. : les constantes et fonctions implicites dans ces notations dépendent a priori de constantes au sens ci-dessus.

Pour $\sigma: K \hookrightarrow \mathbb{C}$, notons $\|s\|_{\infty, \sigma}=\sup \left\{\|s(\xi)\|_{\text {cub }, \sigma} ; \xi \in A_{\sigma}(\mathbb{C})\right\}$. Une comparaison avec la norme hermitienne $\|s\|_{\overline{H_{n}}, \sigma}$ (introduite au paragraphe 3.1) donne l'existence d'une constante $c>0$, qui ne dépend que de $(A, L)$, telle que

$$
\|s\|_{\infty, \sigma} \leq n^{c}\|s\|_{\overline{H_{n}}, \sigma}
$$

(lemme de Gromov, voir [GS, § 5.2.3]).

Lemme 3.8. Soit $\sigma: K \hookrightarrow \mathbb{C}$ un plongement complexe. Soient s un élément de $H^{0}\left(A_{K}, L_{K}^{\otimes n}\right) \otimes_{\sigma} \mathbb{C}$, $\vartheta$ la fonction thêta associée et $\mathbf{e}$ une base orthonormée de $t_{A_{\sigma}}$. Pour tout $r>0$, tout $\tau \in \mathbb{N}^{g}$ et tout $z \in t_{A_{\sigma}}$ on $a:$

$$
\left|\frac{1}{\tau !} D_{\mathbf{e}}^{\tau} \vartheta(z)\right| \exp \left(-\frac{\pi}{2} n\|z\|_{L, \sigma}^{2}\right) \leq \frac{\|s\|_{\infty, \sigma}}{r^{|\tau|}} \exp \left(\frac{\pi}{2} n\left(g r^{2}+2 \sqrt{g} r\|z\|_{L, \sigma}\right)\right) .
$$

Démonstration. En notant $e_{1}, \ldots, e_{g}$ les vecteurs de la base $\mathbf{e}$, les inégalités de Cauchy pour les fonctions holomorphes donnent

$$
\begin{aligned}
\left|\frac{1}{\tau !} D_{\mathbf{e}}^{\tau} \vartheta(z)\right| & \leq \frac{1}{r|\tau|} \sup \left\{|\vartheta(z+\xi)| ; \xi=\xi_{1} e_{1}+\cdots+\xi_{g} e_{g} \in t_{A_{\sigma}},\left|\xi_{i}\right|=r\right\} \\
& \leq \frac{1}{r|\tau|} \sup \left\{|\vartheta(z+\xi)| ;\|\xi\|_{L, \sigma} \leq \sqrt{g} r\right\} .
\end{aligned}
$$

La formule $|\vartheta(z+\xi)|=\left\|s\left(\exp _{A_{\sigma}}(z+\xi)\right)\right\|_{\mathrm{cub}, \sigma} \exp \left(\pi n\|z+\xi\|_{L, \sigma}^{2} / 2\right)$ et l'inégalité triangulaire permettent de conclure.

Corollaire 3.9. Avec les notations du lemme précédent, on a la majoration suivante, pour tout nombre réel $\gamma>0$ :

$$
\left|\frac{1}{\tau !} D_{\mathbf{e}}^{\tau} \vartheta(z)\right| \exp \left(-\frac{\pi}{2} n\|z\|_{L, \sigma}^{2}\right) \leq\left(\frac{\pi n \sqrt{g}}{\gamma}\left(\|z\|_{L, \sigma}+\sqrt{\gamma / 2 \pi n}\right)\right)^{|\tau|} e^{\gamma}\|s\|_{\infty, \sigma} .
$$

Démonstration. Appliquons le lemme précédent avec

de sorte que l'on ait

$$
r=\frac{\frac{2 \gamma}{\pi n \sqrt{g}}}{\|z\|_{L, \sigma}+\sqrt{\|z\|_{L, \sigma}^{2}+\frac{2 \gamma}{\pi n}}},
$$

$$
\frac{\pi}{2} n\left(g r^{2}+2 \sqrt{g} r\|z\|_{L, \sigma}\right)=\gamma .
$$

La majoration

entraîne alors le corollaire.

$$
\frac{1}{r} \leq \frac{\pi n \sqrt{g}}{\gamma}\left(\|z\|_{L, \sigma}+\sqrt{\gamma /(2 \pi n)}\right)
$$


Rappelons qu'étant donné un fibré adélique hermitien $\overline{\mathrm{E}}$ sur un corps de nombres $\mathrm{K}$, la hauteur $h_{\overline{\mathrm{E}}}(\xi)$ d'un élément $\xi \in\left(\mathrm{E} \otimes_{\mathrm{K}} \overline{\mathrm{K}}\right) \backslash\{0\}$ est la moyenne

$$
h_{\overline{\mathrm{E}}}(\xi)=\frac{1}{\left[\mathrm{~K}^{\prime}: \mathbb{Q}\right]} \sum_{v \text { place de } \mathrm{K}^{\prime}}\left[\mathrm{K}_{v}^{\prime}: \mathbb{Q}\right] \log \|\xi\|_{\overline{\mathrm{E}}_{\mathrm{K}^{\prime}}, v}
$$

où $\mathrm{K}^{\prime}$ est une extension finie de $\mathrm{K}$ sur laquelle $\xi$ est défini et $\overline{\mathrm{E}_{\mathrm{K}^{\prime}}}$ le fibré adélique hermitien sur $\mathrm{K}^{\prime}$ obtenu à partir de $\overline{\mathrm{E}}$ par extension des scalaires. Dans la suite, nous notons $\log ^{+} a:=\max \{0, \log a\}$ pour un nombre réel strictement positif $a$

Proposition 3.10. Notons $\rho$ le plus grand des rangs $\rho_{\sigma}$ lorsque $\sigma$ parcourt les plongements de $K$ au-dessus de $\sigma_{0}$. Il existe une section non nulle $s \in H^{0}\left(A, L^{\otimes n}\right) \otimes_{k} \bar{K}$ de hauteur relative $\grave{a} \bar{H}_{n, \alpha}$ plus petite que

$$
\frac{\rho\left[k_{\sigma_{0}}: \mathbb{R}\right]}{\nu D}\left(\log ^{+} \alpha+T+\left(g T+T_{1}\right) \log ^{+}\left(\frac{\pi n \sqrt{g}}{T}\left(S_{1}\|u\|_{L, \sigma_{0}}+\sqrt{T /(2 \pi n)}\right)\right)\right)+o(T) .
$$

Démonstration. D'après le lemme de Siegel approché absolu du $\S 4.1$ de [Ga3], appliqué à $\bar{H}_{n, \alpha}$, il existe $s \in\left(H^{0}\left(A_{K}, L_{K}^{\otimes n}\right) \otimes_{K} \bar{K}\right) \backslash\{0\}$ dont la hauteur $h_{\bar{H}_{n, \alpha}}(s)$ est plus petite que

$$
\sum_{\sigma \mid \sigma_{0} \text { ou } \overline{\sigma_{0}}} \frac{\rho_{\sigma}}{\nu[K: \mathbb{Q}]}\left(\log \max \left\{1,\left\|U_{\sigma}\right\|\right\}+\log \sqrt{1+\alpha^{2}}\right)+\frac{1}{2} \log \nu-\widehat{\mu}\left(\overline{H_{n}}\right),
$$

où $\left\|U_{\sigma}\right\|=\max \left\{\left|U_{\sigma} X\right|_{2} ;|X|_{2}=1\right\}$ est la norme d'opérateur de la matrice $U_{\sigma}$ (de rang $\rho_{\sigma}$ ). Notons tout de suite que la pente de $\overline{H_{n}}$ entre dans le $o(T)$ de l'énoncé par la formule rappelée au paragraphe 3.1. Il nous faut maintenant majorer la norme de $U_{\sigma}$ (qui est aussi celle de $U_{\bar{\sigma}}$ ) pour $\sigma \mid \sigma_{0}$. D'après le corollaire 3.9 avec $\gamma=T$ on a, pour tous $(m, \tau) \in \Upsilon$ et $i \in\{1, \ldots, \nu\}$ :

$$
\left|U_{\sigma}[(m, \tau), i]\right| \leq \max \left\{1, \frac{\pi n \sqrt{g}}{T}\left(S_{1}\|u\|_{L, \sigma_{0}}+\sqrt{T / 2 \pi n}\right)\right\}^{g T+T_{1}} e^{T}\left\|s_{i}\right\|_{\infty, \sigma_{0}} .
$$

Par le lemme de Gromov rappelé au début de ce paragraphe, il existe une constante $c>0$ telle que $\max _{1 \leq i \leq \nu}\left\|s_{i}\right\|_{\infty, \sigma_{0}} \leq n^{c}$. Ainsi la norme de $U_{\sigma}$, qui est naturellement plus petite que $(\nu \operatorname{Card} \Upsilon)^{1 / 2} \max _{(m, \tau), i}\left|U_{\sigma}[(m, \tau), i]\right|$, est inférieure à

$$
(\nu \operatorname{Card} \Upsilon)^{1 / 2} \max \left\{1, \frac{\pi n \sqrt{g}}{T}\left(S_{1}\|u\|_{L, \sigma_{0}}+\sqrt{T / 2 \pi n}\right)\right\}^{g T+T_{1}} e^{T} n^{c} .
$$

Le logarithme de la quantité $(\nu \text { Card } \Upsilon)^{1 / 2} n^{c}$ est négligeable devant $T$ car

$$
\log (\operatorname{Card} \Upsilon)=O(\log T), \quad \log (\nu)=\log \left(n^{g} h^{0}(A, L)\right)=O(\log n)=O(\log T) .
$$

Il ne reste plus qu'à noter $\rho / \nu=O(1)$ (proposition 3.7) et

$$
\sum_{\sigma \mid \sigma_{0} \text { ou } \overline{\sigma_{0}}} \rho_{\sigma}=\left[k_{\sigma_{0}}: \mathbb{R}\right] \sum_{\sigma \mid \sigma_{0}} \rho_{\sigma} \leq\left[k_{\sigma_{0}}: \mathbb{R}\right][K: k] \rho
$$

pour conclure.

3.7. Jet de la section. Dans toute la suite, nous noterons $s$ la section auxiliaire donnée par la proposition 3.10 et $K^{\prime}$ une extension finie de $K$ sur laquelle $s$ est défini. D'après le lemme de multiplicité (proposition 3.3), il existe un couple $(m, \ell) \in\{-g S, \ldots, g S\} \times\{0,1, \ldots, g T\}$, que l'on peut choisir minimal pour l'ordre lexicographique, tel que $s$ ne s'annule pas en $m p$ à l'ordre $\ell$. À l'instar de [GR2, p. 375] et comme au paragraphe 3.1, l'on peut définir le jet d'une section de la manière suivante. Notons $\mathcal{J}$ le faisceau d'idéaux sur $\mathcal{A}$ défini par la section neutre (immersion fermée) $\varepsilon: \operatorname{Spec} \mathcal{O}_{K} \rightarrow \mathcal{A}$ et $\tau_{m}: \mathcal{A} \rightarrow \mathcal{A}$ la translation par $\varepsilon_{m p}$. Par définition l'élément $\tau_{m}^{*} s$ appartient à $H^{0}\left(\mathcal{A}, \mathcal{J}^{\ell} \otimes_{\mathcal{O}_{\mathcal{A}}} \tau_{m}^{*} \mathcal{L}_{n}\right) \otimes_{\mathcal{O}_{K}} K^{\prime}$ et son image dans le quotient

$$
\varepsilon^{*}\left(\mathcal{J}^{\ell} / \mathcal{J}^{\ell+1} \otimes_{\mathcal{O}_{\mathcal{A}}} \tau_{m}^{*} \mathcal{L}_{n}\right) \otimes_{\mathcal{O}_{K}} K^{\prime} \simeq\left(S^{\ell}\left(t_{\mathcal{A}}^{\vee}\right) \otimes_{\mathcal{O}_{K}} \varepsilon_{m p}^{*} \mathcal{L}_{n}\right) \otimes_{\mathcal{O}_{K}} K^{\prime}=: F
$$


définit le jet de $s$ à l'ordre $\ell$ au point $m p$ le long de $t_{A}$. Mentionnons que l'identification de $\varepsilon^{*}\left(\mathcal{J}^{\ell} / \mathcal{J}^{\ell+1}\right)$ avec la puissance symétrique $\ell$-ème du cotangent $t_{\mathcal{A}}^{v}$ provient de la régularité de $\mathcal{J}$, elle-même conséquence de la lissité de $\mathcal{A} \rightarrow \operatorname{Spec} \mathcal{O}_{K}$ en $\varepsilon$. Dans la suite, nous noterons jet ${ }^{\ell} s(m p)$ cette image, non nulle par définition de $(m, \ell)$.

Comme nous l'avons évoqué au paragraphe 3.1 , les $\mathcal{O}_{K}$-modules $t_{\mathcal{A}}$ et $\varepsilon_{m p}^{*} \mathcal{L}$ possèdent des structures de fibrés adéliques hermitiens qui induisent une telle structure sur $F$ par dualité, puissance symétrique et produit tensoriel (voir [GR1, § 2.5 et 2.6]). Nous disposons alors de la

Proposition 3.11. La pente maximale $\widehat{\mu}_{\max }(\bar{F})$ est plus petite que

$$
\ell\left((g+1)\left(h(A)+\frac{1}{2} \log h^{0}(A, L)\right)+2 g^{5} \log 2+2 \log g\right)+n m^{2} \widehat{h}_{L}(p) .
$$

Démonstration. Par la proposition 5.7 de [Ga2] et l'invariance par extension des scalaires de la pente maximale, on a $\widehat{\mu}_{\max }(\bar{F})=\widehat{\mu}_{\max }\left(S^{\ell}\left(\overline{t_{A_{K}}^{\mathrm{V}}}\right)\right)+\widehat{\operatorname{deg}} \varepsilon_{m p}^{*} \overline{\mathcal{L}_{n}}$. Par la proposition 8.4 de [GR1], la première quantité est majorée par $\ell\left(\widehat{\mu}_{\max }\left(\overline{t_{A_{K}}^{v}}\right)+2 \log g\right)$. La proposition 3.10 de [GR2] fournit alors la majoration de la pente maximale

$$
\widehat{\mu}_{\max }\left(\overline{t_{A_{K}}^{v}}\right) \leq(g+1)\left(h(A)+\frac{1}{2} \log h^{0}(A, L)\right)+2 g^{5} \log 2 .
$$

Quant au degré d'Arakelov de $\varepsilon_{m p}^{*} \overline{\mathcal{L}_{n}}$, il est égal à $n$ fois la hauteur de Néron-Tate de $m p$ (voir $\S 3.1$ ) et l'on conclut avec la quadraticité de $\widehat{h}_{L}(\cdot)$.

La définition de la pente maximale et le fait que jet ${ }^{\ell} s(m p) \neq 0$ impliquent

$$
-\widehat{\mu}_{\max }(\bar{F}) \leq h_{\bar{F}}\left(\operatorname{jet}^{\ell} s(m p)\right)
$$

où la hauteur du jet est celle relative à $\bar{F}$ :

$h_{\bar{F}}\left(\operatorname{jet}^{\ell} s(m p)\right)=\frac{1}{\left[K^{\prime}: \mathbb{Q}\right]} \sum_{\sigma: K^{\prime} \hookrightarrow \mathbb{C}} \log \left\|\operatorname{jet}^{\ell} s(m p)\right\|_{\bar{F}, \sigma}+\frac{1}{\left[K^{\prime}: \mathbb{Q}\right]} \sum_{v \nmid \infty}\left[K_{v}^{\prime}: \mathbb{Q}_{v}\right] \log \left\|\operatorname{jet}^{\ell} s(m p)\right\|_{\bar{F}, v}$

(la première somme porte sur les plongements complexes de $K^{\prime}$ et la seconde sur les places ultramétriques).

Nous allons majorer chacune des normes qui apparaissent dans cette expression, obtenant ainsi une borne pour la hauteur du jet que nous comparerons à la minoration de l'opposé de la pente maximale de $\bar{F}$ donnée par la proposition 3.11. Nous distinguerons les places archimédiennes, ultramétriques et les plongements au-dessus de $\sigma_{0}$ ou $\overline{\sigma_{0}}$ en lesquels nous ferons des estimations fines des normes du jet, en séparant les cas périodique et non périodique.

3.8. Estimations générales. Commençons par les estimations ultramétriques du jet, particulièrement simples à énoncer.

Proposition 3.12. Pour toute place ultramétrique $v$ de $K^{\prime}$, on a $\left\|\operatorname{jet}^{\ell} s(m p)\right\|_{\bar{F}_{, v}} \leq\|s\|_{\bar{H}_{n, \alpha}, v}$.

Démonstration. Elle est identique à celle du paragraphe 6.7.1 de [GR2], en remplaçant simplement la section $s$ de cet article par $\tau_{m}^{*} s$.

Les estimations aux places archimédiennes sont un peu plus techniques à établir. Étant donné un plongement complexe $\sigma: K^{\prime} \hookrightarrow \mathbb{C}$, notons $r\left(A_{\sigma}, L_{\sigma}\right)$ le rayon de recouvrement de la variété abélienne complexe polarisée $\left(A_{\sigma}, L_{\sigma}\right)$ :

$$
r\left(A_{\sigma}, L_{\sigma}\right)=\sup \left\{\operatorname{dist}_{\sigma}\left(z, \Omega_{A_{\sigma}}\right) ; z \in t_{A_{\sigma}}\right\}
$$

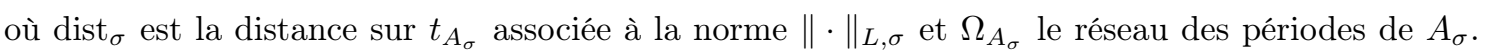
Soient $\vartheta_{\sigma}: t_{A_{\sigma}} \rightarrow \mathbb{C}$ la fonction holomorphe associée à $s$ et $\omega_{\sigma}$ un logarithme de $\sigma(m p)$. Le jet de 
$\vartheta_{\sigma}$ au point $\omega_{\sigma}$ s'exprime en termes d'une base $\mathbf{f}_{\sigma}=\left(f_{1, \sigma}, \ldots, f_{g, \sigma}\right)$ de $t_{A_{\sigma}}$ et de sa base duale $\left(f_{1, \sigma}^{\vee}, \ldots, f_{g, \sigma}^{\vee}\right)$ :

$$
\text { jet }^{\ell} \vartheta_{\sigma}\left(\omega_{\sigma}\right)=\sum_{|\tau|=\ell} \frac{1}{\tau !} D_{\mathbf{f}_{\sigma}}^{\tau} \vartheta_{\sigma}\left(\omega_{\sigma}\right)\left(f_{1, \sigma}^{\vee}\right)^{\tau_{1}} \cdots\left(f_{g, \sigma}^{\vee}\right)^{\tau_{g}}
$$

(dans cette somme $\left.\tau=\left(\tau_{1}, \ldots, \tau_{g}\right) \in \mathbb{N}^{g}\right)$. Cette quantité ne dépend pas du choix de la base $\mathbf{f}_{\sigma}$. La structure hermitienne sur la puissance symétrique $S^{\ell}\left(t_{A_{\sigma}}^{\vee}\right)$ induite par $\left(t_{A_{\sigma}},\|\cdot\|_{L, \sigma}\right)$ est la métrique quotient de celle du produit tensoriel $\left(t_{A_{\sigma}}^{v}\right)^{\otimes \ell}$. Ainsi, lorsque $\mathbf{f}_{\sigma}$ est une base orthonormée, la norme au carré du vecteur $\left(f_{1, \sigma}^{\vee}\right)^{\tau_{1}} \cdots\left(f_{g, \sigma}^{\vee}\right)^{\tau_{g}}$ est égale à $\tau_{1} ! \cdots \tau_{g} ! / \ell !$ et, par la formule donnée au $\S 3.1$, la norme relative à $\sigma$ du jet de $s$ à l'ordre $\ell$ au point $m p$ a pour expression

$$
\| \text { jet }^{\ell} s(m p) \|_{\bar{F}, \sigma}=\left(\sum_{\substack{\tau \in \mathbb{N}^{g} \\|\tau|=\ell}}\left|\frac{1}{\tau !} D_{\mathbf{f}_{\sigma}}^{\tau} \vartheta_{\sigma}\left(\omega_{\sigma}\right)\right|^{2} \frac{\tau_{1} ! \cdots \tau_{g} !}{\ell !}\right)^{1 / 2} \exp \left(-\frac{\pi}{2} n\left\|\omega_{\sigma}\right\|_{L, \sigma}^{2}\right)
$$

(qui ne dépend pas du choix de $\omega_{\sigma}$ parmi les logarithmes de $\sigma(m p)$ ). Nous avons donc en particulier

$$
\left\|\operatorname{jet}^{\ell} s(m p)\right\|_{\bar{F}, \sigma} \leq\left(\begin{array}{c}
\ell+g-1 \\
g-1
\end{array}\right)^{1 / 2} \max _{\substack{\tau \in \mathbb{N}^{g} \\
|\tau|=\ell}}\left|\frac{1}{\tau !} D_{\mathbf{f}_{\sigma}}^{\tau} \vartheta_{\sigma}\left(\omega_{\sigma}\right)\right| \exp \left(-\frac{\pi}{2} n\left\|\omega_{\sigma}\right\|_{L, \sigma}^{2}\right) .
$$

Le coefficient binomial est majoré par $(2 g T)^{g}$. De plus, par définition de $r\left(A_{\sigma}, L_{\sigma}\right)$, le point $\sigma(m p) \in$ $A_{\sigma}(\mathbb{C})$ possède un logarithme $\omega_{\sigma} \in t_{A_{\sigma}}$ vérifiant $\left\|\omega_{\sigma}\right\|_{L, \sigma} \leq r\left(A_{\sigma}, L_{\sigma}\right)$. Le corollaire 3.9 (avec $\gamma=T$ ) et le lemme de Gromov du paragraphe 3.6 (en notant $\|s\|_{\bar{H}_{n}, \sigma} \leq\|s\|_{\bar{H}_{n, \alpha}, \sigma}$ ) conduisent alors au résultat suivant.

Proposition 3.13. Il existe une constante $c>0$, ne dépendant que de $(A, L)$, telle que, pour tout plongement complexe $\sigma: K^{\prime} \hookrightarrow \mathbb{C}$, la norme $\|$ jet $^{\ell} s(m p) \|_{\bar{F}, \sigma}$ du jet est plus petite que

$$
(n T)^{c} e^{T} \max \left\{1, \frac{\pi n \sqrt{g}}{T}\left(r\left(A_{\sigma}, L_{\sigma}\right)+\sqrt{\frac{T}{2 \pi n}}\right)\right\}^{g T}\|s\|_{\bar{H}_{n, \alpha}, \sigma} .
$$

3.9. Extrapolation analytique. Nous allons dans ce paragraphe donner une majoration fine des normes $\|$ jet $^{\ell} s(m p) \|_{\bar{F}, \sigma}$ du jet pour $\sigma: K^{\prime} \hookrightarrow \mathbb{C}$ qui prolonge $\sigma_{0}$ ou $\overline{\sigma_{0}}$. L'invariance par conjugaison complexe de ces normes permet de supposer $\sigma \mid \sigma_{0}$ dans la suite de cette partie.

3.9.1. Cas non périodique.

Proposition 3.14. Supposons que l'on soit dans le cas non périodique. Il existe une constante $c>0$ telle que la norme $\left\|\mathrm{jet}^{\ell} s(m p)\right\|_{\bar{F}, \sigma}$ soit majorée par

$$
\begin{aligned}
(n T)^{c}\|s\|_{\bar{H}_{n, \alpha}, \sigma}[ & \left(\frac{1}{E}\right)^{\left(2 S_{0}+1\right)(T+1)} \times \max \left\{1, \frac{\pi n \sqrt{g}}{T}\left(E g S\|u\|_{L, \sigma_{0}}+\sqrt{\frac{T}{2 \pi n}}\right)\right\}^{g T} \\
& \times\left(1+\frac{\varepsilon_{0}^{2}}{2,9 g^{2}(g+1)^{2 g}}\right)^{S_{0}(T+1)} \exp \left(T+\frac{\pi}{2} n g^{2} S^{2} E^{2}\|u\|_{L, \sigma_{0}}^{2}\right) \\
& \left.+2^{g T} \alpha^{-1} \max \left\{1,\|u\|_{L, \sigma_{0}}\right\}^{T}\left(\frac{1}{\cos \pi \epsilon}\right)^{T+1}\left(\frac{g S}{S_{0}}\right)^{\left(2 S_{0}+1\right)(T+1)}\left(\operatorname{sh}\left(\pi S_{0}\right)\right)^{T+1}\right] .
\end{aligned}
$$

Démonstration. Soient $\vartheta$ la fonction thêta associée à $s$ et $\tau \in \mathbb{N}^{g}$ de longueur $\ell$. Posons

$$
f(z):=\frac{1}{\tau !} D_{\mathbf{f}}^{\tau} \vartheta(z u) .
$$


Par l'estimation (6) (avec $\omega_{\sigma}=m u$ ), le jet de $s$ en $m p$ est relié aux différentes valeurs $f(m)$ lorsque $\tau$ varie. Ce sont ces valeurs que nous allons maintenant évaluer. Par le principe du maximum, on a

$$
\left|\frac{1}{\tau !} D_{\mathbf{f}}^{\tau} \vartheta(m u)\right|=|f(m)| \leq \sup _{|z|=g S}|f(z)|=|f|_{g S} .
$$

Nous allons majorer ce dernier supremum au moyen du lemme d'interpolation analytique donné par la proposition 2.1. Soient $h$ et $j$ deux entiers tels que $0 \leq h \leq T$ et $|j| \leq S_{0}$. Par choix de la base $\mathbf{f}$ (voir le début du paragraphe 3.3) on a $u=\|u\|_{L, \sigma_{0}} f_{g}$, d'où :

$$
\frac{f^{(h)}(z)}{h !}=\frac{\|u\|_{L, \sigma_{0}}^{h}}{\tau ! h !} D_{\mathbf{f}}^{\tau+(0, \ldots, 0, h)} \vartheta(z u) .
$$

Par suite, en notant $\tau^{\prime}=\tau+(0, \ldots, 0, h)$ et en observant que $\tau^{\prime} ! \leq 2^{\ell+h} \tau ! h !$ :

$$
\left|\frac{f^{(h)}(j)}{2^{h} h !}\right| \leq 2^{\ell} \max \left\{1,\|u\|_{L, \sigma_{0}}\right\}^{h}\left|\frac{D_{\mathbf{f}}^{\tau^{\prime}} \vartheta(j u)}{\tau^{\prime} !}\right| .
$$

D'après la définition de la norme $\|\cdot\|_{\bar{H}_{n, \alpha}, \sigma}$ (noter que $\left(j, \tau^{\prime}\right) \in \Upsilon$ ), nous obtenons ainsi :

$$
\begin{aligned}
\sum_{\substack{-S_{0} \leq j \leq S_{0} \\
0 \leq h \leq T}} \frac{\left|f^{(h)}(j)\right|}{2^{h} h !} \leq( & +1)\left(2 S_{0}+1\right) 2^{g T} \alpha^{-1} \max \left\{1,\|u\|_{L, \sigma_{0}}\right\}^{T} \\
& \times \exp \left(\frac{\pi}{2} n S_{0}^{2}\|u\|_{L, \sigma_{0}}^{2}\right)\|s\|_{\bar{H}_{n, \alpha}, \sigma} .
\end{aligned}
$$

Par ailleurs, le corollaire 3.9 avec $\gamma=T$ donne, pour tout nombre réel $R>0$ :

$$
|f|_{R} \leq \max \left\{1, \frac{\pi n \sqrt{g}}{T}\left(R\|u\|_{L, \sigma_{0}}+\sqrt{T / 2 \pi n}\right)\right\}^{\ell} \exp \left(T+\frac{\pi}{2} n R^{2}\|u\|_{L, \sigma_{0}}^{2}\right)\|s\|_{\infty, \sigma} .
$$

L'inégalité de Gromov donne alors une borne en fonction de la norme de $s$ relative à $\bar{H}_{n, \alpha}$. Appliquons maintenant la proposition 2.1 avec $r=g S, R=E r, S_{0}+1$ au lieu de $S, T+1$ au lieu de $T, a=S_{0}$ et $\varepsilon$ le nombre réel fixé au $\S 3.4$. En regroupant les estimations précédentes, la norme $\|$ jet $^{\ell} s(m p) \|_{\bar{F}, \sigma}$ du jet est majorée par

$$
\begin{aligned}
(n T)^{c}[ & \left(\frac{1}{E}\right)^{T+1}\left(\frac{E^{2}\left(1+\frac{\left(S_{0}+1\right)\left(2 S_{0}+1\right)}{6 g^{2} S^{2}}\right)}{E^{4}+\frac{\left(S_{0}+1\right)\left(2 S_{0}+1\right)}{6 g^{2} S^{2}}}\right)^{S_{0}(T+1)} \exp \left(T+\frac{\pi}{2} n g^{2} S^{2} E^{2}\|u\|_{L, \sigma_{0}}^{2}\right) \\
& \times \max \left\{1, \frac{\pi n \sqrt{g}}{T}\left(E g S\|u\|_{L, \sigma_{0}}+\sqrt{T / 2 \pi n}\right)\right\}^{\ell} \\
& \left.+2^{g T} \alpha^{-1} \max \left\{1,\|u\|_{L, \sigma_{0}}\right\}^{T}\left(\frac{1}{\cos \pi \epsilon}\right)^{T+1}\left(\frac{g S}{S_{0}}\right)^{\left(2 S_{0}+1\right)(T+1)}\left(\operatorname{sh}\left(\pi S_{0}\right)\right)^{T+1}\right]\|s\|_{\bar{H}_{n, \alpha}, \sigma},
\end{aligned}
$$

où $c$ est une nouvelle constante ne dépendant pas de $T$ (mais elle dépend de $(A, L)$ et des paramètres $\left.D, E, S_{0}, \varepsilon\right)$. Stricto sensu, cette borne n'est démontrée que si $|m| \geq S_{0}$ car nous avons remplacé $\exp \left(S_{0}^{2}-m^{2}\right)$ par 1. Néanmoins elle reste valable lorsque $|m|<S_{0}$ car

$$
|f(m)| \exp \left(-\frac{\pi}{2} n\|m u\|_{L, \sigma_{0}}^{2}\right) \leq \alpha^{-1}\|s\|_{\bar{H}_{n, \alpha}, \sigma}
$$

par définition de la norme $\|\cdot\|_{\bar{H}_{n, \alpha}, \sigma}$. Il reste à simplifier le majorant du jet et plus particulièrement le quotient

$$
\frac{E^{2}\left(1+\frac{\left(S_{0}+1\right)\left(2 S_{0}+1\right)}{6 g^{2} S^{2}}\right)}{E^{4}+\frac{\left(S_{0}+1\right)\left(2 S_{0}+1\right)}{6 g^{2} S^{2}}} .
$$


Pour cela, nous minorons le dénominateur par $E^{4}$. En utilisant $S_{0} \leq \widetilde{S}_{0}$, la définition de $\widetilde{S}_{0}$ et $2(\xi+1) /(2 \xi+1) \leq 1+1 /(2 \xi)$ pour $\xi=S_{0}$, nous majorons

par

$$
\frac{\left(S_{0}+1\right)\left(2 S_{0}+1\right)}{6 g^{2} S^{2}}=\frac{\left(2 S_{0}+1\right)^{2}}{6 g^{2}(2 S+1)^{2}}\left(2+\frac{1}{S}\right)^{2}\left(\frac{S_{0}+1}{2 S_{0}+1}\right)
$$

$$
\frac{\varepsilon_{0}^{2}}{3 g^{2}(g+1)^{2 g}}\left(1+\frac{1}{2 S}\right)^{2}\left(1+\frac{1}{2 S_{0}}\right) \leq \frac{\varepsilon_{0}^{2}}{2,9 g^{2}(g+1)^{2 g}}
$$

la dernière inégalité provenant de $S \geq S_{0} \geq 50$.

3.9.2. Cas périodique. Les majorations fines des normes du jet en les plongements $\sigma \mid \sigma_{0}$ ou $\sigma \mid \overline{\sigma_{0}}$ sont apportées dans le cas périodique par le résultat suivant.

Proposition 3.15. Supposons que l'on soit dans le cas périodique. Il existe une constante $c>0$ telle que la norme $\|$ jet $^{\ell} s(m p) \|_{\bar{F}, \sigma}$ soit majorée par

$$
\begin{aligned}
&(n T)^{c}\left(\frac{\max \left\{1,\|u\|_{L, \sigma_{0}}\right\}}{u_{g}}\right)^{g T} \times\|s\|_{\bar{H}_{n, \alpha}, \sigma} \\
& \times\left[\left(\frac{4}{3}\right)^{g S\left(T_{0}+1\right)}\left(\frac{1}{E}\right)^{(2 g S+1)\left(T_{0}+1\right)} \exp \left(T+\frac{\pi}{2} n E^{2}(g S+1)^{2}\|u\|_{L, \sigma_{0}}^{2}\right)\right. \\
& \quad \times \max \left\{1, \frac{\pi n \sqrt{g}}{T}\left(E(g S+1)\|u\|_{L, \sigma_{0}}+\sqrt{T / 2 \pi n}\right)\right\}^{g T} \\
&\left.\quad+2^{g T} \alpha^{-1} \max \left\{1,\|u\|_{L, \sigma_{0}}\right\}^{T_{0}}\left(\frac{\operatorname{sh}(\pi(g S+1))}{\cos \pi \epsilon}\right)^{T_{0}+1} \exp \left(\frac{\pi}{2} n g^{2} S^{2}\|u\|_{L, \sigma_{0}}^{2}\right)\right] .
\end{aligned}
$$

Démonstration. En reprenant la base orthonormée $\mathbf{f}$ introduite au paragraphe 3.3 et les coordonnées $\left(u_{1}, \ldots, u_{g}\right)$ de $u$ dans cette base, on a

$$
u_{g}>0 \quad \text { et } \quad f_{g}=\frac{1}{u_{g}}\left(u-u_{1} f_{1}-\cdots-u_{g-1} f_{g-1}\right) .
$$

La base duale de la base $\left(f_{1}, \ldots, f_{g-1}, u\right)$ de $t_{A_{\sigma_{0}}}$ s'écrit, pour $\xi=\xi_{1} f_{1}+\cdots+\xi_{g} f_{g} \in t_{A_{\sigma_{0}}}$,

$$
\forall i \in\{1, \ldots, g-1\}, \quad f_{i}^{\vee}(\xi)=\xi_{i}-\xi_{g} \frac{u_{i}}{u_{g}} \quad \text { et } \quad u^{\vee}(\xi)=\frac{\xi_{g}}{u_{g}} .
$$

En particulier les normes des vecteurs de cette base duale vérifient

$$
\forall i \in\{1, \ldots, g-1\}, \quad\left\|f_{i}^{\vee}\right\|_{\overline{t_{A_{K}}}, \sigma_{0}}=\left(1+\left(\frac{\left|u_{i}\right|}{u_{g}}\right)^{2}\right)^{1 / 2} \quad \text { et } \quad\left\|u^{\vee}\right\|_{\overline{t_{A_{K}}}}, \sigma_{0}=\frac{1}{u_{g}} .
$$

Elles sont donc plus petites que $\max \left\{1,\|u\|_{L, \sigma_{0}}\right\} / u_{g}$. En notant $\vartheta$ la fonction thêta associée à $s$ et à l'instar de la majoration (6) (en omettant l'exponentielle), la norme $\|$ jet $^{\ell} s(m p) \|_{\bar{F}, \sigma}$ est alors inférieure à

$$
\begin{aligned}
& \left(\begin{array}{c}
\ell+g-1 \\
g-1
\end{array}\right)^{1 / 2}\left(\frac{\max \left\{1,\|u\|_{L, \sigma_{0}}\right\}}{u_{g}}\right)^{\ell} \\
& \quad \times \max \left\{\left|\frac{D_{f_{1}}^{\tau_{1}} \cdots D_{f_{g-1}}^{\tau_{g-1}} D_{u}^{\tau_{g}} \vartheta(m u)}{\tau_{1} ! \cdots \tau_{g} !}\right| ;\left(\tau_{1}, \ldots, \tau_{g}\right) \in \mathbb{N}^{g} \text { de longueur } \ell\right\} .
\end{aligned}
$$

Dans le deuxième facteur de cette expression, nous pouvons remplacer $\ell$ par $g T$ car le quotient $\max \left\{1,\|u\|_{L, \sigma_{0}}\right\} / u_{g}$ est plus grand que 1 . Ce sont les dérivées divisées apparaissant dans le maximum que nous allons maintenant majorer. Fixons $\tau=\left(\tau_{1}, \ldots, \tau_{g}\right) \in \mathbb{N}^{g}$ de longueur $\ell$ et posons 
$\tau^{\prime}=\left(\tau_{1}, \ldots, \tau_{g-1}, 0\right)$ puis

$$
f(z):=\frac{1}{\tau^{\prime} !} D_{\mathbf{f}}^{\tau^{\prime}} \vartheta(z u) .
$$

D'après les inégalités de Cauchy pour $f$ on a :

$$
\left|\frac{1}{\tau !} D_{\mathbf{f}}^{\tau^{\prime}} D_{u}^{\tau_{g}} \vartheta(m u)\right|=\left|\frac{1}{\tau_{g} !} f^{\left(\tau_{g}\right)}(m)\right| \leq|f|_{1+|m|} \leq|f|_{1+g S} .
$$

À ce stade, l'idée est encore de majorer $|f|_{1+g S}$ au moyen du lemme d'interpolation obtenu dans la partie 2. Soient $h$ et $j$ deux entiers tels que $0 \leq h \leq T_{0}$ et $|j| \leq g S$. Comme $u=u_{1} f_{1}+\cdots+u_{g} f_{g}$, on a, pour tout $z \in \mathbb{C}$ :

$$
\frac{f^{(h)}(z)}{h !}=\frac{1}{\tau^{\prime} ! h !} D_{\mathbf{f}}^{\tau^{\prime}} D_{u}^{h} \vartheta(z u)=\sum_{\substack{\tau^{\prime \prime} \in \mathbb{N}^{g} \\\left|\tau^{\prime \prime}\right|=h}} u_{1}^{\tau_{1}^{\prime \prime}} \cdots u_{g}^{\tau_{g}^{\prime \prime}} \frac{1}{\tau^{\prime} ! \tau^{\prime \prime} !} D_{\mathbf{f}}^{\tau^{\prime}+\tau^{\prime \prime}} \vartheta(z u) .
$$

Utilisons maintenant les majorations

$$
\left|u_{i}\right| \leq\|u\|_{L, \sigma_{0}}, \quad \frac{\left(\tau^{\prime}+\tau^{\prime \prime}\right) !}{\tau^{\prime} ! \tau^{\prime \prime} !}=\prod_{i=1}^{g} \frac{\left(\tau_{i}^{\prime}+\tau_{i}^{\prime \prime}\right) !}{\tau_{i}^{\prime} ! \tau_{i}^{\prime \prime} !} \leq \prod_{i=1}^{g} 2^{\tau_{i}^{\prime}+\tau_{i}^{\prime \prime}} \leq 2^{\ell+h}
$$

ainsi que la majoration

$$
\left|\frac{1}{\left(\tau^{\prime}+\tau^{\prime \prime}\right) !} D_{\mathbf{f}}^{\tau^{\prime}+\tau^{\prime \prime}} \vartheta(j u)\right| \leq \alpha^{-1}\|s\|_{\bar{H}_{n, \alpha}, \sigma} \exp \left(\frac{\pi}{2} n\|j u\|_{L, \sigma_{0}}^{2}\right)
$$

provenant de la définition de la norme $\|\cdot\|_{\bar{H}_{n, \alpha}, \sigma}$ (noter que $\left(j, \tau^{\prime}+\tau^{\prime \prime}\right) \in \Upsilon$ ). L'expression $\left|f^{(h)}(j)\right| /\left(2^{h} h\right.$ !) est alors plus petite que

$$
\left(\begin{array}{c}
g+h-1 \\
g-1
\end{array}\right) 2^{\ell} \max \left\{1,\|u\|_{L, \sigma_{0}}\right\}^{h} \alpha^{-1} \exp \left(\frac{\pi}{2} n j^{2}\|u\|_{L, \sigma_{0}}^{2}\right)\|s\|_{\bar{H}_{n, \alpha}, \sigma} .
$$

En utilisant $\sum_{h=0}^{T_{0}}\left(\begin{array}{c}g+h-1 \\ g-1\end{array}\right)=\left(\begin{array}{c}T_{0}+g \\ g\end{array}\right) \leq\left(1+T_{0}\right)^{g}$, nous obtenons

$$
\begin{array}{r}
\left|\sum_{\substack{g S \leq \leq j \leq g S \\
0 \leq h \leq T_{0}}} \frac{f^{(h)}(j)}{2^{h} h !}\right| \leq(2 g S+1)\left(T_{0}+1\right)^{g} 2^{g T} \max \left\{1,\|u\|_{L, \sigma_{0}}\right\}^{T_{0}} \\
\times \alpha^{-1} \exp \left(\frac{\pi}{2} n g^{2} S^{2}\|u\|_{L, \sigma_{0}}^{2}\right)\|s\|_{\bar{H}_{n, \alpha}, \sigma} .
\end{array}
$$

Par ailleurs, en utilisant l'estimation archimédienne générale des dérivées du corollaire 3.9 (avec $\gamma=T$, comme dans le cas non périodique) et le lemme de Gromov, nous avons l'existence d'une constante $c>0$ telle que, pour tout nombre réel $R>0$ :

$$
|f|_{R} \leq n^{c}\left(\frac{\pi n \sqrt{g}}{T}\left(R\|u\|_{L, \sigma_{0}}+\sqrt{T / 2 \pi n}\right)\right)^{\left|\tau^{\prime}\right|} \exp \left(T+\frac{\pi}{2} n R^{2}\|u\|_{L, \sigma_{0}}^{2}\right)\|s\|_{\bar{H}_{n, \alpha}, \sigma}
$$

Appliquons alors le lemme d'interpolation 2.1 avec $r=a=g S+1, R=E r, g S+1$ au lieu de $S$ et $T_{0}+1$ au lieu de $T$ et $\varepsilon$ le nombre réel fixé au $\S 3.4$. Quitte à augmenter $c$, la norme du vecteur 
jet $^{\ell} s(m p)$ est alors plus petite que

$$
\begin{aligned}
& (n T)^{c}\left(\frac{\max \left\{1,\|u\|_{L, \sigma_{0}}\right\}}{u_{g}}\right)^{g T} \times\|s\|_{\bar{H}_{n, \alpha}, \sigma} \\
& \times\left[\left(\frac{1}{E}\right)^{T_{0}+1}\left(\frac{E^{2}\left(1+\frac{2 g S+1}{6(g S+1)}\right)}{E^{4}+\frac{2 g S+1}{6(g S+1)}}\right)^{\left(T_{0}+1\right) g S} \exp \left(T+\frac{\pi}{2} n E^{2}(g S+1)^{2}\|u\|_{L, \sigma_{0}}^{2}\right)\right. \\
& \times \max \left\{1, \frac{\pi n \sqrt{g}}{T}\left(E(g S+1)\|u\|_{L, \sigma_{0}}+\sqrt{T / 2 \pi n}\right)\right\}^{\ell} \\
& \left.+2^{g T} \alpha^{-1} \max \left\{1,\|u\|_{L, \sigma_{0}}\right\}^{T_{0}}\left(\frac{\operatorname{sh}(\pi(g S+1))}{\cos \pi \epsilon}\right)^{T_{0}+1} \exp \left(\frac{\pi}{2} n g^{2} S^{2}\|u\|_{L, \sigma_{0}}^{2}\right)\right] \text {. }
\end{aligned}
$$

On conclut au moyen de l'encadrement $0 \leq 2 g S+1 \leq 2(g S+1)$ qui implique :

$$
\frac{E^{2}\left(1+\frac{2 g S+1}{6(g S+1)}\right)}{E^{4}+\frac{2 g S+1}{6(g S+1)}} \leq \frac{4}{3 E^{2}} .
$$

3.10. Majoration de la hauteur du jet. Les estimations de la norme du jet en les différentes places de $K^{\prime}$ établies aux paragraphes 3.8 et 3.9 vont nous permettre d'obtenir une majoration de la hauteur du jet.

3.10.1. Cas non périodique.

Proposition 3.16. Avec les notations $P$ et $Q_{\epsilon}$ introduites au $\S 3.4$, on a, dans le cas non périodique :

$$
\begin{aligned}
\frac{h_{\bar{F}}\left(\mathrm{jet}^{\ell} s(m p)\right)}{\left[k_{\sigma_{0}}: \mathbb{R}\right]} \leq & \frac{1-\varepsilon_{0}}{D} \log P+\frac{\varepsilon_{0}}{D} \max \left\{\log P, \log Q_{\epsilon}\right\}+\frac{D-1}{D} T+\frac{\varepsilon_{0}}{D} T \\
& +\frac{g T}{D} \sum_{\sigma: k \hookrightarrow \mathbb{C}} \log ^{+}\left(\pi x \sqrt{g} r\left(A_{\sigma}, L_{\sigma}\right)+\sqrt{\pi g x / 2}\right) \\
& +\frac{\varepsilon_{0}}{D}(g+1) T \log ^{+}\left(\pi x \sqrt{g} S_{0}\|u\|_{L, \sigma_{0}}+\sqrt{\pi g x / 2}\right)+o(T) .
\end{aligned}
$$

Démonstration. Avec les définitions (2) et (3) de $P$ et $Q_{\epsilon}$, et en utilisant l'inégalité $n / T<x$, l'estimation de la proposition 3.14 se réécrit :

$$
\left\|\operatorname{jet}^{\ell} s(m p)\right\|_{\bar{F}_{, \sigma}} \leq(n T)^{c}\left(P+\alpha^{-1} Q_{\epsilon}\right)\|s\|_{\bar{H}_{n, \alpha}, \sigma}=2 P(n T)^{c}\|s\|_{\bar{H}_{n, \alpha}, \sigma}
$$

pour tout $\sigma: K^{\prime} \hookrightarrow \mathbb{C}$ qui prolonge $\sigma_{0}$ ou $\overline{\sigma_{0}}$. En combinant cette estimation avec celles des propositions 3.12 et 3.13 , la hauteur du jet vérifie :

$$
\begin{aligned}
& h_{\bar{F}}\left(\operatorname{jet}^{\ell} s(m p)\right) \leq h_{\bar{H}_{n, \alpha}}(s)+\frac{\left[k_{\sigma_{0}}: \mathbb{R}\right]}{D} \log (2 P)+\frac{D-\left[k_{\sigma_{0}}: \mathbb{R}\right]}{D} T \\
& +\frac{g T}{\left[K^{\prime}: \mathbb{Q}\right]} \sum_{\substack{\sigma: K^{\prime} \hookrightarrow \mathbb{C} \\
\sigma \nmid \sigma_{0} \text { et } \sigma \nmid \sigma_{0}}} \log ^{+}\left(\frac{\pi n \sqrt{g}}{T}\left(r\left(A_{\sigma}, L_{\sigma}\right)+\sqrt{T / 2 \pi n}\right)\right)+O(\log (T n)) .
\end{aligned}
$$

Dans la somme, nous pouvons oublier les conditions sur $\sigma$ quitte à ajouter des termes positifs et, comme $r\left(A_{\sigma}, L_{\sigma}\right)$ ne dépend que de $\sigma_{\mid k}$, nous pouvons faire la moyenne sur les plongements complexes de $k$. Nous allons utiliser la proposition 3.10 pour majorer $h_{\bar{H}_{n, \alpha}}(s)$. Remarquons tout 
d'abord que $P \geq E^{-\left(2 S_{0}+1\right)(T+1)}$, donc $\alpha \leq Q_{\epsilon} E^{\left(2 S_{0}+1\right)(T+1)} \leq c^{T}$, où $c>0$ ne dépend pas de $T$. On en déduit $\log ^{+} \alpha=O(T)$. Observons maintenant que la proposition 3.7 fournit l'estimation :

$$
\frac{\rho}{\nu} \leq(g+1)^{g}\left(\frac{2 S_{0}+1}{2 S+1}\right)(1+o(1)) \leq(g+1)^{g}\left(\frac{2 \widetilde{S}_{0}+1}{2 S+1}\right)(1+o(1))=\varepsilon_{0}(1+o(1)) .
$$

Comme $n / T<x=O(1)$ d'après (1), la proposition 3.10 permet de majorer la hauteur $h_{\bar{H}_{n, \alpha}}(s)$ de $s$ par

$$
\frac{\varepsilon_{0}\left[k_{\sigma_{0}}: \mathbb{R}\right]}{D}\left(\log ^{+} \alpha+T+(g+1) T \log ^{+}\left(\pi x \sqrt{g} S_{0}\|u\|_{L, \sigma_{0}}+\sqrt{\pi g x / 2}\right)\right)+o(T) .
$$

Il ne reste plus qu'à remplacer $\alpha$ par sa définition $\alpha=Q_{\epsilon} / P$ pour conclure.

3.10.2. Cas périodique. Rappelons que $\eta=\varepsilon_{0}$ si $g=1$ et $\eta=g^{g} \varepsilon_{0}\left(1-\varepsilon_{0}\right)^{-1}$ si $g \geq 2$. Le choix de $\varepsilon_{0}$ dans le cas périodique équivaut à $\eta=0,06$.

Proposition 3.17. Supposons que l'on soit dans le cas périodique. Alors, avec les notations $P$ et $Q_{\epsilon}$ introduites au $\S 3.4$, on $a$ :

$$
\begin{aligned}
\frac{h_{\bar{F}}\left(\mathrm{jet}^{\ell} s(m p)\right)}{\left[k_{\sigma_{0}}: \mathbb{R}\right]} \leq & \frac{1-\eta}{D} \log P+\frac{\eta}{D} \max \left\{\log P, \log Q_{\epsilon}\right\}+\frac{g T}{D} \log \left(\frac{\max \left\{1,\|u\|_{L, \sigma_{0}}\right\}}{u_{g}}\right) \\
& +\frac{D-1}{D} T+\frac{\eta}{D} T+\frac{g T}{D} \sum_{\sigma: k \hookrightarrow \mathbb{C}} \log ^{+}\left(\pi x \sqrt{g} r\left(A_{\sigma}, L_{\sigma}\right)+\sqrt{\pi g x / 2}\right) \\
& +\frac{\eta}{D}\left(g+\varepsilon_{0}\right) T \log ^{+}\left(\pi x g \sqrt{g} S\|u\|_{L, \sigma_{0}}+\sqrt{\pi g x / 2}\right)+o(T) .
\end{aligned}
$$

Démonstration. Avec les définitions (4) et (5) de $P$ et $Q_{\epsilon}$, et en utilisant l'inégalité $n / T<x$, l'estimation de la proposition 3.15 se réécrit :

$$
\left\|\operatorname{jet}^{\ell} s(m p)\right\|_{\bar{F}, \sigma} \leq 2 P(n T)^{c}\left(\frac{\max \left\{1,\|u\|_{L, \sigma_{0}}\right\}}{u_{g}}\right)^{g T}\|s\|_{\bar{H}_{n, \alpha}, \sigma}
$$

pour $\sigma: K^{\prime} \hookrightarrow \mathbb{C}$ qui prolonge $\sigma_{0}$ ou $\overline{\sigma_{0}}$ et en notant que $P+\alpha^{-1} Q_{\epsilon}=2 P$. En combinant cette

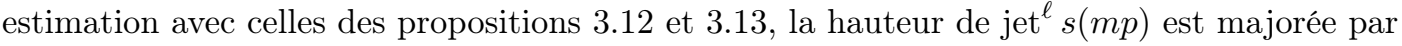

$$
\begin{aligned}
& \frac{\left[k_{\sigma_{0}}: \mathbb{R}\right]}{D} \log (2 P)+\frac{g T\left[k_{\sigma_{0}}: \mathbb{R}\right]}{D} \log \left(\frac{\max \left\{1,\|u\|_{L, \sigma_{0}}\right\}}{u_{g}}\right)+\frac{D-\left[k_{\sigma_{0}}: \mathbb{R}\right]}{D} T+h_{\bar{H}_{n, \alpha}}(s) \\
& +\frac{g T}{\left[K^{\prime}: \mathbb{Q}\right]} \sum_{\substack{\sigma: K^{\prime} \hookrightarrow \mathbb{C} \\
\sigma \nmid \sigma_{0} \text { et } \sigma \nmid \bar{\sigma}_{0}}} \log ^{+}\left(\pi x \sqrt{g}\left(r\left(A_{\sigma}, L_{\sigma}\right)+\sqrt{1 / 2 \pi x}\right)\right)+O(\log (T n)) .
\end{aligned}
$$

Comme dans le cas précédent, la dernière somme divisée par $\left[K^{\prime}: \mathbb{Q}\right]$ peut être remplacée par la moyenne sur tous les plongements complexes de $k$. Utilisons la proposition 3.10 pour majorer $h_{\bar{H}_{n, \alpha}}(s)$. Tout d'abord, par la proposition 3.7 nous avons $\rho / \nu \leq \eta(1+o(1))$. D'autre part, la définition de $P$ montre que $\alpha \leq Q_{\epsilon} E^{(2 g S+1)\left(T_{0}+1\right)}$, d'où $\log ^{+} \alpha=O(T)$. Comme en outre $n / T<$ $x=O(1)$ d'après l'encadrement (1), la proposition 3.10 donne alors :

$$
h_{\bar{H}_{n, \alpha}}(s) \leq \frac{\eta\left[k_{\sigma_{0}}: \mathbb{R}\right]}{D}\left(\log ^{+} \alpha+T+\left(g+\varepsilon_{0}\right) T \log ^{+}\left(\pi x g \sqrt{g} S\|u\|_{L, \sigma_{0}}+\sqrt{\pi g x / 2}\right)\right)+o(T) .
$$

Nous reportons alors dans la majoration ci-dessus de la hauteur du jet puis nous remplaçons $\alpha$ par sa définition $\alpha=Q_{\epsilon} / P$. 
3.11. Conclusion dans le cas $g \geq 2$. Nous sommes maintenant en mesure d'achever la démontration du théorème 3.1 lorsque $g \geq 2$ (le cas $g=1$ sera traité au paragraphe 3.12). Nous commençons par regrouper quelques lemmes préliminaires au $\S 3.11 .1$. Nous minorons alors la hauteur du jet au $\S 3.11 .2$ puis l'on procède à quelques réductions au $\S 3.11$.3. La minoration du jet obtenue, jointe à la majoration du paragraphe précédent, permet alors d'obtenir le théorème 3.1, aux paragraphes 3.11.4 (cas non périodique) et 3.11.5 (cas périodique).

3.11.1. Lemmes préliminaires. Nous rassemblons ici quelques lemmes généraux qui seront utilisés dans la suite. Commençons par deux énoncés élémentaires qui interviendront plusieurs fois.

Lemme 3.18. Soient $a>0, b \geq 0$ et $c>1$ trois nombres réels. Si $\xi>0$ est un nombre réel tel que $\xi \leq a(b+\log \xi)$ alors

$$
\xi<a\left(1+\frac{\log c}{c-1}\right) \max \{c, b+\log a\}
$$

Démonstration. Quitte à remplacer $\xi$ par $\xi e^{b}$ et $a$ par $a e^{b}$ nous pouvons supposer $b=0$. Posons $\alpha=\max \{c, \log a\}$. Si $\xi=e^{\alpha}$ alors l'hypothèse $\xi \leq a \log \xi$ implique $\xi \leq a \alpha$ puis le résultat. Sinon, on a $\log \left(\xi^{1 / \alpha}\right)<\xi^{1 / \alpha} e^{-1}$ et de l'hypothèse $\xi \leq a \log \xi$ l'on tire

$$
\xi<\left(\frac{a \alpha}{e}\right)^{\frac{\alpha}{\alpha-1}} \text { puis } \xi<\frac{a \alpha}{\alpha-1} \log \left(\frac{a \alpha}{e}\right) .
$$

Le choix de $\alpha$ conduit à $\xi<a \alpha\left(1+\frac{\log \alpha}{\alpha-1}\right)$ et l'on conclut en utilisant $\alpha \geq c$ et la décroissance de la fonction $\xi \mapsto(\log \xi) /(\xi-1)$ sur $] 1,+\infty[$.

Lemme 3.19. Soient $x_{1}, \ldots, x_{n}$ des nombres réels positifs. Alors

$$
\frac{1}{n} \sum_{i=1}^{n} \log \max \left\{1, x_{i}\right\} \leq \max \left\{1, \log \left(\frac{1}{n} \sum_{i=1}^{n} x_{i}\right)\right\} .
$$

Démonstration. Nous pouvons supposer $x_{1} \geq \cdots \geq x_{n}$. Soient $m$ le nombre de $x_{i}$ supérieurs à 1 et $M$ la moyenne de tous les $x_{i}$. Si $m=0$, il n'y a rien à démontrer. Si $m \geq 1$, on écrit $1 / n=(m / n) \times(1 / m)$ et on utilise la concavité du logarithme pour avoir

$$
\frac{1}{n} \sum_{i=1}^{n} \log \max \left\{1, x_{i}\right\} \leq \frac{m}{n} \log \left(\frac{1}{m}\left(x_{1}+\cdots+x_{m}\right)\right) \leq \frac{m}{n} \log \left(\frac{n M}{m}\right) .
$$

Comme $1+\log \xi \leq \xi$ pour tout $\xi \geq 1$, on a

$$
\frac{m}{n} \log \left(\frac{n M}{m}\right) \leq \frac{m}{n}\left(\frac{n}{m}-1\right)+\frac{m}{n} \log M=\frac{n-m}{n}+\frac{m}{n} \log M .
$$

La dernière somme convexe est plus petite que $\max \{1, \log M\}$ et la proposition est démontrée.

Nous utiliserons également une majoration du rayon de recouvrement d'une variété abélienne que nous avons défini au paragraphe 3.8. Soient $(A, L)$ une variété abélienne polarisée sur un corps de nombres $k$ et $\sigma: k \hookrightarrow \mathbb{C}$ un plongement archimédien. Le réseau des périodes $\Omega_{A_{\sigma}}$ de l'espace euclidien $\left(t_{A_{\sigma}},\|\cdot\|_{L, \sigma}\right)$ est de rang $2 g$. Le réseau polaire $\Omega_{A_{\sigma}}^{*}=\left\{\xi \in t_{A_{\sigma}} ; \forall \omega \in \Omega_{A_{\sigma}}, \operatorname{Re}\langle\xi, \omega\rangle \in \mathbb{Z}\right\}$ vérifie $h^{0}(A, L) i \Omega_{A_{\sigma}}^{*} \subset \Omega_{A_{\sigma}}$ (voir la démonstration de la proposition 3.5 de [GR3]) et, en particulier, son rayon de recouvrement est plus grand que celui de $\Omega_{A_{\sigma}}$ divisé par $h^{0}(A, L)$. En utilisant le théorème 2.2 de $[\mathrm{Ba}]$, nous obtenons ainsi

$$
r\left(A_{\sigma}, L_{\sigma}\right) \leq \frac{g h^{0}(A, L)}{\rho\left(A_{\sigma}, L_{\sigma}\right)}
$$


où $\rho\left(A_{\sigma}, L_{\sigma}\right)$ est le premier minimum du réseau $\Omega_{A_{\sigma}}$, c'est-à-dire aussi le diamètre d'injectivité de $\left(A_{\sigma}, L_{\sigma}\right)$. Le lemme matriciel d'Autissier $[\mathrm{Au}]$ dans sa variante donnée par la proposition 3.6 de [GR2] en fournit une minoration sous la forme :

$$
\frac{1}{[k: \mathbb{Q}]} \sum_{\sigma: k \hookrightarrow \mathbb{C}} \frac{1}{\rho\left(A_{\sigma}, L_{\sigma}\right)^{2}} \leq 11 \max \left\{1, h(A), \log \operatorname{deg}_{L} A\right\} .
$$

La convexité de $\xi \mapsto \xi^{2}$ et l'égalité $h^{0}(A, L)=\left(\operatorname{deg}_{L} A\right) / g$ ! conduisent alors au

Lemme 3.20. On a

$$
\frac{1}{[k: \mathbb{Q}]} \sum_{\sigma: k \hookrightarrow \mathbb{C}} r\left(A_{\sigma}, L_{\sigma}\right) \leq \frac{1}{(g-1) !}\left(\operatorname{deg}_{L} A\right)\left(11 \max \left\{1, h(A), \log \operatorname{deg}_{L} A\right\}\right)^{1 / 2} .
$$

3.11.2. Minoration de la hauteur du jet.

Proposition 3.21. La hauteur de jet ${ }^{\ell} s(m p)$ relative à $\bar{F}$ est supérieure à

$$
-n g^{2} S^{2} \widehat{h}_{L}(p)-g T\left(2 g^{5} \log 2+2 \log g+(g+1)\left(\max \{0, h(A)\}+\frac{1}{2} \log h^{0}(A, L)\right)\right) .
$$

Démonstration. Il s'agit simplement de combiner la proposition 3.11 et l'inégalité qui suit sa démonstration, en tenant compte de $|m| \leq g S$ et $\ell \leq g T$.

3.11.3. Réductions. Afin de démontrer le théorème 3.1 , nous pouvons supposer que la norme de $u$ est petite. Plus précisément, nous disposons du résultat suivant (la constante $c_{0}(g)$ est celle définie dans le théorème 3.1) :

Lemme 3.22. Pour démontrer le théorème 3.1, nous pouvons supposer que les conditions suivantes sont satisfaites :

$$
x\|u\|_{L, \sigma_{0}}^{2} \leq \frac{(2 S+1)^{1 / g}}{c_{0}(g)} \quad \text { et } \quad \log \|u\|_{L, \sigma_{0}} \leq \frac{\log h^{0}(A, L)}{2 g} .
$$

Démonstration. Supposons d'abord que l'on ait $x\|u\|_{L, \sigma_{0}}^{2}>(2 S+1)^{1 / g} / c_{0}(g)$. Comme $x \leq$ $\left((2 S+1) / \operatorname{deg}_{L} A\right)^{1 / g}$ par (1), la quantité $\left(\operatorname{deg}_{L} A\right)^{1 / g} \log E$ est plus petite que

$$
(2 S+1)^{1 / g}(\log E) x^{-1} \leq c_{0}(g)\|u\|_{L, \sigma_{0}}^{2} \log E \leq c_{0}(g)\left(D \widehat{h}_{L}(p)+\frac{\pi}{2} E^{2}\|u\|_{L, \sigma_{0}}^{2}\right),
$$

qui s'avère être une majoration meilleure que celle du théorème. Supposons maintenant que $2 g \log \|u\|_{L, \sigma_{0}}>\log h^{0}(A, L)$. Alors on a

$$
\left(\operatorname{deg}_{L} A\right)^{1 / g} \log E \leq g !^{1 / g}\|u\|_{L, \sigma_{0}}^{2} \log E \leq g\left(D \widehat{h}_{L}(p)+\frac{\pi}{2} E^{2}\|u\|_{L, \sigma_{0}}^{2}\right),
$$

qui est de nouveau une majoration meilleure que celle du théorème.

Dorénavant et jusqu'à la fin du paragraphe 3.11 , nous supposerons que les conditions du lemme 3.22 sont satisfaites. Nous posons également :

$$
h:=\max \left\{1, h(A), \log \operatorname{deg}_{L} A\right\} .
$$

Le résultat suivant va permettre de majorer différentes quantités apparaissant dans les bornes de la hauteur du jet données par les propositions 3.16 et 3.17.

Lemme 3.23. Soit $E^{\prime} \geq 1$ un nombre réel. On a les majorations suivantes :

(i)

$$
\frac{1}{D} \sum_{\sigma: k \hookrightarrow \mathbb{C}} \log ^{+}\left(\pi x \sqrt{g} r\left(A_{\sigma}, L_{\sigma}\right)+\sqrt{\frac{\pi x g}{2}}\right) \leq 3,09+\log \left(\sqrt{h}\left(\operatorname{deg}_{L} A\right)^{1-1 / g} S^{1 / g}\right)
$$


(ii)

$$
\log ^{+}\left(\pi x \sqrt{g} E^{\prime}(g S+1)\|u\|_{L, \sigma_{0}}+\sqrt{\frac{\pi g x}{2}}\right) \leq-9,52+\log E^{\prime}+\left(1+\frac{1}{g}\right) \log S .
$$

Comme on le verra dans la démonstration, ce lemme ne dépend pas du cas périodique ou non dans lequel on se trouve. De plus, l'on peut bien évidemment remplacer le terme $E^{\prime}(g S+1)$ dans le point (ii) par un minorant quelconque, comme $S_{0}, g S, g E S$ (en prenant $E^{\prime}=E$ ).

Démonstration. Pour obtenir ces majorations, le principe est de mettre en facteur une quantité dominante après avoir donné une majoration simple de l'expression à évaluer, en utilisant la borne (1) de $x$. Pour (i), nous utilisons les lemmes 3.19 et 3.20 :

$$
\begin{aligned}
\frac{1}{D} \sum_{\sigma: k \hookrightarrow \mathbb{C}} \log ^{+}\left(\pi x \sqrt{g} r\left(A_{\sigma}, L_{\sigma}\right)+\sqrt{\frac{\pi x g}{2}}\right) & \leq \max \left\{1, \log \left(\frac{\pi x \sqrt{g}}{D} \sum_{\sigma: k \hookrightarrow \mathbb{C}} r\left(A_{\sigma}, L_{\sigma}\right)+\sqrt{\frac{\pi x g}{2}}\right)\right\} \\
& \leq \max \left\{1, \log \left(\frac{\pi g \sqrt{g}}{g !} x \operatorname{deg}_{L} A \sqrt{11 h}+\sqrt{\frac{\pi x g}{2}}\right)\right\} .
\end{aligned}
$$

Majorons alors $g \sqrt{g} / g$ ! par $\sqrt{2}$ puis $x$ par $(2 S+1)^{1 / g}\left(\operatorname{deg}_{L} A\right)^{-1 / g}$. Il vient

$$
\begin{aligned}
& \pi x \sqrt{22 h} \operatorname{deg}_{L} A+\sqrt{\frac{\pi x g}{2}} \\
& \leq\left(\pi \sqrt{22}+\frac{\sqrt{\pi g / 2}}{\left(\operatorname{deg}_{L} A\right)^{1-1 / 2 g}(2 S+1)^{1 / 2 g}}\right) \sqrt{h}(2 S+1)^{1 / g}\left(\operatorname{deg}_{L} A\right)^{1-1 / g} .
\end{aligned}
$$

En minorant $\operatorname{deg}_{L} A$ par 2 et $2 S+1$ par $2 g^{g}$ et en utilisant

$$
(2 S+1)^{1 / g}=\left(2+\frac{1}{S}\right)^{1 / g} S^{1 / g} \leq\left(2+\frac{1}{100}\right)^{1 / 2} S^{1 / g},
$$

nous obtenons

$$
\pi x \sqrt{22 h} \operatorname{deg}_{L} A+\sqrt{\frac{\pi x g}{2}} \leq\left(\pi \sqrt{22}+\frac{\sqrt{\pi}}{2 \sqrt{2}}\right)\left(2+\frac{1}{100}\right)^{1 / 2} \sqrt{h} S^{1 / g}\left(\operatorname{deg}_{L} A\right)^{1-1 / g} .
$$

La partie (i) du lemme résulte alors de l'évaluation numérique des deux premières parenthèses dont le produit est plus petit que exp $(3,09)$. Pour (ii), nous commençons par remplacer la norme de $u$ par la première borne du lemme 3.22. Nous majorons ensuite $\sqrt{g x}$ par $\sqrt{e} S^{1 / 2 g}$ en utilisant $x \leq\left((2 S+1) / \operatorname{deg}_{L} A\right)^{1 / g}$ et $\operatorname{deg}_{L} A \geq g ! \geq e(g / e)^{g}$. Nous écrivons alors

$$
\pi x \sqrt{g} E^{\prime}(g S+1)\|u\|_{L, \sigma_{0}}+\sqrt{\frac{\pi g x}{2}} \leq \sqrt{e}\left(\frac{\pi\left(g+S^{-1}\right)\left(2+S^{-1}\right)^{1 / 2 g}}{\sqrt{c_{0}(g)}}+\frac{\sqrt{\pi / 2}}{S^{1+1 / 2 g}}\right) E^{\prime} S^{1+1 / g} .
$$

Il reste à voir que la quantité devant $E^{\prime} S^{1+1 / g}$ est bien plus petite que $\exp (-9,52)$. Pour cela, nous minorons $S$ par la partie entière $s$ de $248 \times 2^{9} \log 2$ et nous observons que la fonction $g \mapsto$ $\left(g+s^{-1}\right) / \sqrt{c_{0}(g)}$ est décroissante pour $g \geq 2$, ce qui permet de la majorer par sa valeur en 2 . Dans le dénominateur $S^{1+1 / 2 g}=S \times S^{1 / 2 g}$ nous minorons aussi $S^{1 / 2 g}$ par $\sqrt{2}$. L'exponentielle de $-9,52$ résulte alors d'une estimation numérique.

La fin de la démonstration du théorème 3.1 se scinde maintenant en deux cas. 
3.11.4. Conclusion dans le cas non périodique. Le lemme suivant fournit l'inégalité clef qui

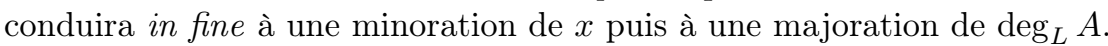

Lemme 3.24. Posons

$$
\aleph_{1}=\frac{1-\varepsilon_{0}}{2 D} \log \left(1+\frac{\varepsilon_{0}^{2}}{2,9 g^{2}(g+1)^{2 g}}\right)+\frac{\varepsilon_{0}}{D} \log \left(\frac{g(g+1)^{g}}{\varepsilon_{0}}\left(1+\frac{3}{2 S_{0}}\right)\right)+\frac{\pi \varepsilon_{0}}{2 D}
$$

$e t$

$$
\aleph_{2}=\beta+\gamma \log S,
$$

ò̀

$$
\begin{gathered}
\beta=\frac{1-\varepsilon_{0}}{D} g \log E+\frac{g}{2} \log h+h\left(\frac{\varepsilon_{0}}{2 g}+\frac{3 g^{2}}{2}+\frac{5 g}{2}\right) \\
+2 g^{6} \log 2+2 g \log g-\frac{g(g+1)}{2} \log g !-6 g
\end{gathered}
$$

et

$$
\gamma=g+2+\varepsilon_{0}\left(1+\frac{1}{g}\right)
$$

Alors on $a$ :

$$
\frac{1-\varepsilon_{0}}{D}\left(2 S_{0}+1\right) \log E \leq x g^{2} S^{2}\left(\widehat{h}_{L}(p)+\frac{\pi}{2 D} E^{2}\|u\|_{L, \sigma_{0}}^{2}\right)+\left(2 S_{0}+1\right) \aleph_{1}+\aleph_{2} .
$$

Démonstration. La proposition 3.16 et le lemme 3.23 fournissent la majoration :

$$
\begin{aligned}
\frac{h_{\bar{F}}\left(\text { jet }^{\ell} s(m p)\right)}{\left[k_{\sigma_{0}}: \mathbb{R}\right]} \leq & \frac{1-\varepsilon_{0}}{D} \log P+\frac{\varepsilon_{0}}{D} \max \left\{\log P, \log Q_{\epsilon}\right\}+\frac{D-1}{D} T+\frac{\varepsilon_{0}}{D} T \\
& +g T\left(3,09+\log \left(\sqrt{h}\left(\operatorname{deg}_{L} A\right)^{1-1 / g} S^{1 / g}\right)\right) \\
& +\frac{\varepsilon_{0}}{D}(g+1) T\left(-9,52+\left(1+\frac{1}{g}\right) \log S\right)+o(T) .
\end{aligned}
$$

La définition (2) de $P$, le lemme 3.23 (ii), les majorations $n \leq x T$ et $\varepsilon_{0} \leq 1$ donnent tout d'abord:

$$
\begin{aligned}
\log P \leq & -\left(2 S_{0}+1\right) T \log E+g T \log E+\frac{\pi}{2} x T(g S)^{2} E^{2}\|u\|_{L, \sigma_{0}}^{2}+T-9,52 g T \\
& +S_{0} T \log \left(1+\frac{\varepsilon_{0}^{2}}{2,9 g^{2}(g+1)^{2 g}}\right)+(g+1) T \log S+O(1) \\
\leq & \frac{\pi}{2} x T(g S)^{2} E^{2}\|u\|_{L, \sigma_{0}}^{2}+(g+1) T \log S+O(1) .
\end{aligned}
$$

D'autre part, en revenant à la définition de $Q_{\epsilon}$ on a :

$$
\begin{aligned}
\log Q_{\epsilon} \leq & g T \log 2+T \log ^{+}\|u\|_{L, \sigma_{0}}+T \log \left(\frac{1}{\cos \pi \epsilon}\right) \\
& +\left(2 S_{0}+1\right) T \log \left(\frac{g S}{S_{0}}\right)+T \log \operatorname{sh}\left(\pi S_{0}\right)+O(1) .
\end{aligned}
$$

Remarquons que d'après la minoration de $S_{0}$ donnée par le lemme 3.4 et la définition de $\widetilde{S}$, on a facilement $(g+1) \log \widetilde{S}+\log 4 \leq S_{0}$, d'où

$$
(g+1) \log S \leq(g+1) \log \widetilde{S} \leq \pi S_{0}-\log 4 \leq \log \operatorname{sh}\left(\pi S_{0}\right) .
$$

L'on en déduit :

$$
\begin{aligned}
& \max \left\{\log P, \log Q_{\epsilon}\right\} \leq \frac{\pi}{2} \\
& x T(g S)^{2} E^{2}\|u\|_{L, \sigma_{0}}^{2}+g T \log 2+T \log ^{+}\|u\|_{L, \sigma_{0}}+T \log \left(\frac{1}{\cos \pi \epsilon}\right) \\
&+\left(2 S_{0}+1\right) T \log \left(\frac{g S}{S_{0}}\right)+T \log \operatorname{sh}\left(\pi S_{0}\right)+O(1) .
\end{aligned}
$$


En reportant dans le majorant de $h_{\bar{F}}\left(\right.$ jet $\left.^{\ell} s(m p)\right)$ donné au début de la démonstration et en combinant avec la minoration de cette hauteur donnée par la proposition 3.21 et en minorant $\left[k_{\sigma_{0}}: \mathbb{R}\right]$ par 1 , nous aboutissons à l'inégalité :

$$
\begin{aligned}
\frac{1-\varepsilon_{0}}{D}( & \left.2 S_{0}+1\right) T \log E \\
\leq & x T g^{2} S^{2}\left(\widehat{h}_{L}(p)+\frac{\pi}{2 D} E^{2}\|u\|_{L, \sigma_{0}}^{2}\right)+\frac{1-\varepsilon_{0}}{D} g T \log E+T \\
& +\frac{1-\varepsilon_{0}}{D} S_{0} T \log \left(1+\frac{\varepsilon_{0}^{2}}{2,9 g^{2}(g+1)^{2 g}}\right)+\frac{1-\varepsilon_{0}}{D}((g+1) \log S-9,52 g) T \\
& +\frac{\varepsilon_{0}}{D} g T \log 2+\frac{\varepsilon_{0}}{D} T \log ^{+}\|u\|_{L, \sigma_{0}}+\frac{\varepsilon_{0}}{D} T \log \left(\frac{1}{\cos \pi \epsilon}\right) \\
& +\frac{\varepsilon_{0}}{D}\left(2 S_{0}+1\right) T \log \left(\frac{g S}{S_{0}}\right)+\frac{\varepsilon_{0}}{D} T \log \operatorname{sh}\left(\pi S_{0}\right)+3,09 g T \\
& +g T \log \left(\sqrt{h}\left(\operatorname{deg}_{L} A\right)^{1-1 / g} S^{1 / g}\right)+\frac{\varepsilon_{0}}{D}(g+1) T\left(\left(1+\frac{1}{g}\right) \log S-9,52\right) \\
& +g T\left(2 g^{5} \log 2+2 \log g+(g+1)\left(\max \{0, h(A)\}+\frac{1}{2} \log h^{0}(A, L)\right)\right)+o(T) .
\end{aligned}
$$

Divisons maintenant cette inégalité par $T$, faisons tendre $T$ vers $+\infty$ puis $\epsilon$ vers 0 . Nous obtenons une nouvelle inégalité, dans laquelle nous reportons les majorations suivantes :

$$
\begin{array}{ll}
\star & \frac{1}{D}((g+1) \log S-9,52 g) \leq(g+1) \log S-9,52 g \\
\star & \frac{g S}{S_{0}}=\left(\frac{g S}{2 S+1}\right)\left(\frac{2 S+1}{2 S_{0}+3}\right)\left(\frac{2 S_{0}+3}{S_{0}}\right) \leq \frac{g(g+1)^{g}}{\varepsilon_{0}}\left(1+\frac{3}{2 S_{0}}\right) \\
\star & \log \operatorname{sh}\left(\pi S_{0}\right) \leq \pi S_{0}-\log 2 \leq \frac{\pi}{2}\left(2 S_{0}+1\right)-\log 2 \\
\star & \log \|u\|_{L, \sigma_{0}} \leq\left(\log h^{0}(A, L)\right) / 2 g \leq h / 2 g \quad(\operatorname{lemme} 3.22) \\
\star & h(A) \leq h, \quad \log h^{0}(A, L) \leq h-\log g !, \quad \log \left(\operatorname{deg}_{L} A\right) \leq h .
\end{array}
$$

Il en résulte l'inégalité du lemme mais, avec

$$
\begin{aligned}
& \frac{1-\varepsilon_{0}}{D} g \log E+\frac{g}{2} \log h+h\left(\frac{\varepsilon_{0}}{2 g}+\frac{3 g^{2}}{2}+\frac{5 g}{2}-1\right)+2 g^{6} \log 2 \\
& +2 g \log g-\frac{g(g+1)}{2} \log g !-6,43 g+1+\varepsilon_{0}((g-1) \log 2-9,52)
\end{aligned}
$$

à la place de $\beta$. Nous simplifions alors cette expression en remarquant que $-h+1$ est négatif et que $\varepsilon_{0}((g-1) \log 2-9,52)$ est négatif si $g \leq 14$ et plus petit que $\varepsilon_{0} g \log 2 \leq 0,01 g$ si $g \geq 15$.

Lemme 3.25. On $a$ :

$$
\aleph_{1} \leq \frac{1-\varepsilon_{0}}{4 D} \log E \quad \text { et } \quad \aleph_{2} \leq \frac{1-\varepsilon_{0}}{4 D}\left(2 S_{0}+1\right) \log E .
$$

Démonstration. Pour la majoration de $\aleph_{1}$, nous utilisons $\log (1+\xi) \leq \xi$ :

$$
\frac{D \aleph_{1}}{1-\varepsilon_{0}} \leq \frac{\varepsilon_{0}}{1-\varepsilon_{0}}\left(\frac{\varepsilon_{0}}{5,8 g^{2}(g+1)^{2 g}}+g \log (g+1)+\log g+\log \left(1+\frac{3}{2 S_{0}}\right)+\frac{\pi}{2}-\log \varepsilon_{0}\right) .
$$

Compte tenu de $S_{0} \geq 256$ (lemme 3.4) et de la valeur de $\varepsilon_{0}=0,57 /(4 g \log (g+1)+4 \log g+7,31$ ), nous avons

$$
\frac{\varepsilon_{0}}{5,8 g^{2}(g+1)^{2 g}}+\log \left(1+\frac{3}{2 S_{0}}\right)+\frac{\pi}{2} \leq 1,577
$$


si bien que $D \aleph_{1} /\left(1-\varepsilon_{0}\right)$ est plus petit que $1 / 4$ dès lors que

$$
7,31+4 g \log (g+1)+4 \log g \leq \frac{1}{\varepsilon_{0}}-4 \log \frac{1}{\varepsilon_{0}} .
$$

Comme $\varepsilon_{0}^{-1} \geq 33$, on a $\varepsilon_{0}^{-1}-4 \log \varepsilon_{0}^{-1} \geq \varepsilon_{0}^{-1}(1-4(\log 33) / 33) \geq 0,57 \varepsilon_{0}^{-1}$ et le choix de $\varepsilon_{0}$ permet donc de voir que l'inégalité ci-dessus est satisfaite. Passons maintenant à la majoration de $\aleph_{2}$. Comme $\frac{2 \widetilde{S}_{0}+1}{2 S_{0}+1} \leq \frac{2 S_{0}+3}{2 S_{0}+1} \leq 1+\frac{1}{S_{0}} \leq 1,01$, il suffit de vérifier

$$
\aleph_{2}=\beta+\gamma \log S \leq \frac{1-\varepsilon_{0}}{4 D \times 1,01}\left(2 \widetilde{S}_{0}+1\right) \log E=\frac{\varepsilon_{0}\left(1-\varepsilon_{0}\right)}{4,04 D(g+1)^{g}}(2 S+1) \log E,
$$

ou bien encore, puisque $2 S+1 \geq 2 S$ :

$$
\beta+\gamma \log S \leq \frac{\varepsilon_{0}\left(1-\varepsilon_{0}\right)}{2,02 D(g+1)^{g}} S \log E .
$$

Si l'on pose

$$
\delta=\frac{\varepsilon_{0}\left(1-\varepsilon_{0}\right)}{2,02 D(g+1)^{g}}, \quad a=\frac{\gamma}{\delta \log E} \quad \text { et } \quad b=\frac{\beta}{\gamma},
$$

nous souhaitons montrer que $a(b+\log S) \leq S$. En vertu du lemme 3.18 appliqué avec $c=31,3$, il suffit de vérifier

$$
a\left(1+\frac{\log (31,3)}{30,3}\right) \max \{(31,3), b+\log a\} \leq S .
$$

Dans la suite nous utiliserons

$$
\max \left\{\frac{(1+1 / g)^{g}}{(g \log g) \varepsilon_{0}\left(1-\varepsilon_{0}\right)} ; g \geq 2\right\} \leq 55,41
$$

qui découle de la valeur exacte de $\varepsilon_{0}$ (le maximum est atteint pour $g=2$ ). On a alors

$$
a=\frac{2,02(g+1)^{g}\left(g+2+\varepsilon_{0}\left(1+g^{-1}\right)\right)}{\varepsilon_{0}\left(1-\varepsilon_{0}\right)} \times \frac{D}{\log E} \leq 226,4 g^{g+2}(\log g) \frac{D}{\log E} .
$$

Nous en déduisons

$$
\begin{aligned}
\log a & \leq \log (226,4)+(g+2) \log g+\log \log g+\log \left(\frac{D}{\log E}\right) \\
& \leq 6,37 g(\log g) \max \left\{1, \log \left(\frac{D}{\log E}\right)\right\}
\end{aligned}
$$

puis $a \log a \leq 1443 g^{g+3}(\log g)^{2} M$ où $M$ est le nombre réel défini au début du paragraphe 3.2. Majorons maintenant la quantité $a b=\frac{\beta}{\delta \log E}$. De la formule qui définit $\beta$ (lemme 3.24) et $\log h \leq$ $h / e$, nous déduisons une majoration de $D \beta / \log E$ par

$$
\left(g+\frac{g}{2 e}+\frac{\varepsilon_{0}}{2 g}+\frac{3 g^{2}}{2}+\frac{5 g}{2}+2 g^{6} \log 2+2 g \log g-\frac{g(g+1)}{2} \log g !-6 g\right) M .
$$

Par un calcul numérique, nous obtenons donc

$$
\frac{D \beta}{\log E} \leq 1,42 g^{6} M \quad \text { puis } \quad a b \leq 1,42 g^{6} M \times \frac{2,02(g+1)^{g}}{\varepsilon_{0}\left(1-\varepsilon_{0}\right)} \leq 159 g^{g+7}(\log g) M .
$$

De ces estimations vient :

$$
\max \{(31,3) a, a b+a \log a\} \leq g^{g+7}(\log g) M \max \left\{(31,3 \times 226,4) g^{-5}, 1443(\log g) g^{-4}+159\right\} .
$$

Ce majorant est plus petit que $222 g^{g+7}(\log g) M$. En multipliant par $1+\log (31,3) / 30,3$ nous obtenons l'estimation souhaitée :

$$
a\left(1+\frac{\log (31,3)}{30,3}\right) \max \{(31,3), b+\log a\} \leq 247,3 g^{g+7}(\log g) M \leq \widetilde{S}-1 \leq[\widetilde{S}]=S .
$$


Fin de la démonstration du théorème 3.1. Les lemmes 3.24 et 3.25 entraînent

$$
\frac{1-\varepsilon_{0}}{2 D}\left(2 S_{0}+1\right) \log E \leq x g^{2} S^{2}\left(\widehat{h}_{L}(p)+\frac{\pi}{2 D} E^{2}\|u\|_{L, \sigma_{0}}^{2}\right) .
$$

Puisque $x \leq(2 S+1)^{1 / g}\left(\operatorname{deg}_{L} A\right)^{-1 / g}$, nous en déduisons

$$
\left(\operatorname{deg}_{L} A\right)^{1 / g} \log E \leq \frac{2 g^{2} S^{2}}{1-\varepsilon_{0}} \cdot \frac{(2 S+1)^{1 / g}}{2 S_{0}+1}\left(D \widehat{h}_{L}(p)+\frac{\pi}{2} E^{2}\|u\|_{L, \sigma_{0}}^{2}\right) .
$$

Pour obtenir le théorème 3.1, il reste à simplifier ce majorant et, plus précisément, l'expression devant la parenthèse. On a

ce qui entraîne

$$
2 S_{0}+1 \geq \frac{\varepsilon_{0}(2 S+1)}{1,01(g+1)^{g}}
$$

$$
\begin{aligned}
\frac{2 g^{2} S^{2}}{1-\varepsilon_{0}} \cdot \frac{(2 S+1)^{1 / g}}{2 S_{0}+1} & \leq \frac{2,02 g^{2}(g+1)^{g}}{\left(1-\varepsilon_{0}\right) \varepsilon_{0}} \times \frac{S^{2}}{(2 S+1)^{1-1 / g}} \\
& \leq\left(1,01 g^{g+3} \log g\right)\left(\frac{(1+1 / g)^{g}}{(g \log g) \varepsilon_{0}\left(1-\varepsilon_{0}\right)}\right) S(2 S+1)^{1 / g} .
\end{aligned}
$$

Majorons alors $S(2 S+1)^{1 / g}$ par

$$
248 g^{g+7} \log g \times\left(496 g^{g+7} \log g+1\right)^{1 / g} M^{1+1 / g} \leq 52025 g^{g+8}(\log g) M^{1+1 / g} .
$$

Le théorème 3.1 s'obtient alors en observant $1,01 \times 55,41 \times 52025 \leq 3.10^{6}$.

3.11.5. Conclusion dans le cas périodique. Dans cette partie, nous démontrons le théorème 3.1 dans le cas périodique et lorsque $g \geq 2$. Rappelons que nous avons supposé que les conditions du lemme 3.22 sont satisfaites, que la lettre $h$ désigne $\max \left\{1, h(A), \log _{\operatorname{deg}} A\right\}$ et que, comme dans la proposition 3.17, $\eta=g^{g} \varepsilon_{0}\left(1-\varepsilon_{0}\right)^{-1}=0,06$.

Lemme 3.26. Posons

$$
\aleph_{1}=\frac{(1-\eta) \varepsilon_{0}}{2 D} \log \frac{4}{3}+\frac{\eta \pi \varepsilon_{0}}{2 D} \quad \text { et } \quad \aleph_{2}=\beta+\gamma \log S,
$$

où

$$
\begin{aligned}
\beta= & \frac{1-\eta}{D} g \log E+h\left(\frac{\eta \varepsilon_{0}}{2 g}+\frac{3 g^{2}}{2}+\left(\frac{5}{2}+\frac{1}{e}\right) g\right) \\
& +g(g+1,11) \log \left(2+\frac{1}{S}\right)+g\left((g+5) \log g+2 g^{5} \log 2\right)
\end{aligned}
$$

et

$$
\gamma=g^{2}+2,11 g+2+\eta \varepsilon_{0}\left(1+\frac{1}{g}\right) .
$$

Alors on $a$ :

$$
\frac{1-\eta}{D} \varepsilon_{0}(2 g S+1) \log E \leq x(g S+1)^{2}\left(\widehat{h}_{L}(p)+\frac{\pi}{2 D} E^{2}\|u\|_{L, \sigma_{0}}^{2}\right)+(2 g S+1) \aleph_{1}+\aleph_{2} .
$$

Démonstration. La proposition 3.17 et le lemme 3.23 donnent :

$$
\begin{aligned}
\frac{h_{\bar{F}}\left(\text { jet }^{\ell} s(m p)\right)}{\left[k_{\sigma_{0}}: \mathbb{R}\right]} \leq & \frac{D-1}{D} T+\frac{\eta}{D} T+\frac{g T}{D} \log \left(\frac{\max \left\{1,\|u\|_{L, \sigma_{0}}\right\}}{u_{g}}\right)+\frac{1-\eta}{D} \log P \\
& +\frac{\eta}{D} \max \left\{\log P, \log Q_{\epsilon}\right\}+g T\left(3,09+\log \left(\sqrt{h}\left(\operatorname{deg}_{L} A\right)^{1-1 / g} S^{1 / g}\right)\right) \\
& +\frac{\eta}{D}\left(g+\varepsilon_{0}\right) T\left(\left(1+\frac{1}{g}\right) \log S-9,52\right)+o(T) .
\end{aligned}
$$


La définition (4) de $P$, l'inégalité $n<x T, T_{0}=\varepsilon_{0} T+O(1)$ et le lemme 3.23 (ii) fournissent l'estimation :

$$
\begin{gathered}
\log P \leq-\varepsilon_{0}(2 g S+1) T \log E+g T \log E+\frac{\pi}{2} x T(g S+1)^{2} E^{2}\|u\|_{L, \sigma_{0}}^{2}+T \\
+\varepsilon_{0} g S T \log \frac{4}{3}+(g+1) T \log S-9,52 g T+O(1) .
\end{gathered}
$$

D'autre part, en revenant à la définition (5) de $Q_{\epsilon}$, on a :

$$
\begin{aligned}
\log Q_{\epsilon} \leq & g T \log 2+\varepsilon_{0} T \log ^{+}\|u\|_{L, \sigma_{0}}+\varepsilon_{0} T \log \left(\frac{1}{\cos \pi \epsilon}\right) \\
& +\varepsilon_{0} T \log \operatorname{sh}(\pi(g S+1))+\frac{\pi}{2} x T g^{2} S^{2}\|u\|_{L, \sigma_{0}}^{2}+O(1) .
\end{aligned}
$$

Remarquons maintenant que, puisque $S \geq 0$, on a

$$
\varepsilon_{0} g S \log \frac{4}{3} \leq \varepsilon_{0} \frac{\pi}{2} g S \leq \frac{\varepsilon_{0}}{2}(\pi(g S+1)-\log 4) \leq \frac{\varepsilon_{0}}{2} \log \operatorname{sh}(\pi(g S+1)) .
$$

Comme $S$ est supérieur à $248 g^{g+6}$ on a

$$
(g+1) \log S=\left(1+\frac{1}{g}\right) \frac{\log S}{S} \times g S \leq \frac{3}{2} \times \frac{\log (248)+(g+6) \log g}{248 g^{g+6}} \times g S,
$$

inégalité qui entraîne $(g+1) \log S \leq \varepsilon_{0} \pi g S / 2$. Nous en déduisons

$$
\max \left\{\varepsilon_{0} g S \log \frac{4}{3}+(g+1) \log S, \varepsilon_{0} \log (\operatorname{sh}(\pi(g S+1)))\right\}=\varepsilon_{0} \log \operatorname{sh}(\pi(g S+1)) .
$$

Par suite, en remarquant que $-2 \varepsilon_{0} g S T \log E+g T \log E \leq 0$ et en omettant les termes négatifs dans la majoration ci-dessus de $\log P$, nous trouvons :

$$
\begin{aligned}
\max \left\{\log P, \log Q_{\epsilon}\right\} \leq & \frac{\pi}{2} x T(g S+1)^{2} E^{2}\|u\|_{L, \sigma_{0}}^{2}+g T \log 2+\varepsilon_{0} T \log ^{+}\|u\|_{L, \sigma_{0}} \\
& +\varepsilon_{0} T \log \left(\frac{1}{\cos \pi \epsilon}\right)+\varepsilon_{0} T \log \operatorname{sh}(\pi(g S+1))+O(1) .
\end{aligned}
$$

En reportant ces majorations dans celle de la hauteur du jet obtenue au début de la démonstration, nous obtenons une borne pour cette hauteur que nous comparons à la minoration fournie par la proposition 3.21. En minorant $\left[k_{\sigma_{0}}: \mathbb{R}\right]$ par 1 , il vient :

$$
\begin{aligned}
& \frac{1-\eta}{D} \varepsilon_{0}(2 g S+1) T \log E \\
& \leq x T\left(g^{2} S^{2} \widehat{h}_{L}(p)+\frac{\pi}{2 D}(g S+1)^{2} E^{2}\|u\|_{L, \sigma_{0}}^{2}\right)+\frac{g T}{D} \log \left(\frac{\max \left\{1,\|u\|_{L, \sigma_{0}}\right\}}{u_{g}}\right) \\
& \quad+T+\frac{1-\eta}{D} g T \log E+\frac{1-\eta}{D} \varepsilon_{0} g S T \log \left(\frac{4}{3}\right)+\frac{1-\eta}{D}((g+1) T \log S-9,52 g T) \\
& \quad+\frac{\eta}{D} g T \log 2+\frac{\eta}{D} \varepsilon_{0} T \log ^{+}\|u\|_{L, \sigma_{0}}+\frac{\eta}{D} \varepsilon_{0} T \log \left(\frac{1}{\cos \pi \epsilon}\right)+\frac{\eta}{D} \varepsilon_{0} T \log \operatorname{sh}(\pi(g S+1)) \\
& \quad+3,09 g T+g T \log \left(\sqrt{h}\left(\operatorname{deg}_{L} A\right)^{1-1 / g} S^{1 / g}\right)+\frac{\eta}{D}\left(g+\varepsilon_{0}\right) T\left(\left(1+\frac{1}{g}\right) \log S-9,52\right) \\
& \quad+g T\left(2 g^{5} \log 2+2 \log g+(g+1)\left(\max \{0, h(A)\}+\frac{1}{2} \log h^{0}(A, L)\right)\right)+o(T) .
\end{aligned}
$$

Par ailleurs, grâce à la seconde condition du lemme 3.22 et au lemme 3.19, nous avons

$$
\frac{1}{D} \log \left(\frac{\max \left\{1,\|u\|_{L, \sigma_{0}}\right\}}{u_{g}}\right) \leq \frac{1}{2 D} \log \left(\frac{h^{0}(A, L)^{1 / g}}{u_{g}^{2}}\right) \leq \max \left\{\frac{1}{2}, \log \left(\frac{\left(\operatorname{deg}_{L} A\right)^{1 / g}}{D u_{g}^{2}}\right)^{1 / 2}\right\}
$$


Ce dernier terme est contrôlé au moyen de la proposition 3.5 et il est majoré par

$$
\begin{aligned}
& \log (2 S)+g \log (2 S+1)+\frac{1}{2} \log (131)+(g+3) \log g+\frac{1}{2} \log h+\frac{1}{2} \log \log (2 S+1) \\
& \leq(g+1,11) \log S+(g+1,11) \log \left(2+\frac{1}{S}\right)+\frac{1}{2} \log (131)+(g+3) \log g+\frac{1}{2} \log h
\end{aligned}
$$

(pour la seconde inégalité nous utilisons $\log \log (2 S+1) \leq 0,21 \log (2 S+1)$ qui découle de $2 S+1 \geq$ $\exp (12))$. Au moyen des majorations

$$
\log \operatorname{sh}(\pi(g S+1)) \leq \pi(g S+1), \quad h(A) \leq h, \quad \log h^{0}(A, L) \leq \log \left(\operatorname{deg}_{L} A\right) \leq h,
$$

et en utilisant de nouveau le lemme 3.22 , nous aboutissons à

$$
\begin{aligned}
\frac{1-\eta}{D} \varepsilon_{0} & (2 g S+1) T \log E \\
\leq & x T(g S+1)^{2}\left(\widehat{h}_{L}(p)+\frac{\pi}{2 D} E^{2}\|u\|_{L, \sigma_{0}}^{2}\right)+T+\frac{1-\eta}{D} g T \log E+T g(g+1,11) \log S \\
& +g T\left((g+1,11) \log \left(2+\frac{1}{S}\right)+(g+3) \log g+\frac{1}{2} \log (131)+\frac{1}{2} \log h\right) \\
& +\frac{1-\eta}{D} \varepsilon_{0} g S T \log \left(\frac{4}{3}\right)+\frac{1-\eta}{D}((g+1) T \log S-9,52 g T)+\frac{\eta}{D} g T \log 2 \\
& +\frac{h}{2 g D} \eta \varepsilon_{0} T+\frac{\eta}{D} \varepsilon_{0} T \log \left(\frac{1}{\cos \pi \epsilon}\right)+\frac{\eta}{D} \varepsilon_{0} T \pi(g S+1)+3,09 g T \\
& +\frac{1}{2} g T \log h+(g-1) T h+T \log S+\frac{\eta}{D}\left(g+\varepsilon_{0}\right) T\left(\left(1+\frac{1}{g}\right) \log S-9,52\right) \\
& +g T\left(2 g^{5} \log 2+2 \log g+\frac{3}{2}(g+1) h\right)+o(T) .
\end{aligned}
$$

Dans cette inégalité, majorons le premier terme de la quatrième ligne par

$$
\frac{(1-\eta) \varepsilon_{0}}{2 D}(2 g S+1) T \log (4 / 3)
$$

et, sur la ligne suivante, écrivons $g S+1=\frac{1}{2}(2 g S+1)+\frac{1}{2}$. Ôtons également le facteur $1 / D$ devant la quantité (positive) $(g+1) \log S-9,52 g$ des quatrième et sixième lignes. Divisons par $T$ puis faisons tendre $T$ vers $+\infty$ et $\epsilon$ vers 0 . Nous obtenons alors l'inégalité du lemme avec, à la place de $\beta$, l'expression

$$
\begin{aligned}
& \frac{1-\eta}{D} g \log E+h\left(\frac{\eta \varepsilon_{0}}{2 g}+\frac{3 g^{2}}{2}+\frac{5 g}{2}-1\right)+g \log h+\frac{g}{2} \log (131) \\
& +g(g+1,11) \log \left(2+\frac{1}{S}\right)+g(g+3) \log g+g\left(2 g^{5} \log 2+2 \log g\right) \\
& +\eta g \log 2+3,09 g+1+\frac{\eta \pi \varepsilon_{0}}{2}-9,52(1-\eta) g-9,52 \eta\left(g+\varepsilon_{0}\right) .
\end{aligned}
$$

Nous majorons alors $\log h \operatorname{par} h / e$ et $-h+1$ par 0 . À l'aide de la valeur exacte $\eta=0,06$, nous pouvons calculer la constante numérique devant $g$, plus petite que -3 et il ne reste plus qu'à observer que $(\pi / 2-9,52) \varepsilon_{0} \eta \leq 0$ pour obtenir la constante $\beta$.

Lemme 3.27. On $a$ :

$$
\aleph_{1} \leq \frac{1-\eta}{4 D} \varepsilon_{0} \log E \quad \text { et } \quad \aleph_{2} \leq \frac{1-\eta}{4 D} \varepsilon_{0}(2 g S+1) \log E .
$$


Démonstration. Comme $E \geq e$, la première inégalité est satisfaite si $\aleph_{1} \leq(1-\eta) \varepsilon_{0} /(4 D)$. Compte tenu de la définition de $\aleph_{1}$, cette inégalité équivaut à

$$
\eta\left(2 \pi+1-2 \log \left(\frac{4}{3}\right)\right) \leq 1-2 \log \left(\frac{4}{3}\right),
$$

inégalité qui est vraie puisque $\eta=0,06$. Passons maintenant à la majoration de $\aleph_{2}$. Comme $2 g S+1 \geq 2 g S$, il suffit de vérifier

$$
\beta+\gamma \log S \leq \frac{1-\eta}{2 D} \varepsilon_{0} g S \log E .
$$

Posons

$$
\delta=\frac{(1-\eta) \varepsilon_{0} g}{2 D}, \quad a=\frac{\gamma}{\delta \log E} \quad \text { et } \quad b=\frac{\beta}{\gamma}
$$

si bien qu'il s'agit de voir que $a(b+\log S) \leq S$. Nous allons montrer que

$$
a\left(1+\frac{\log 40}{39}\right) \max \{40, b+\log a\} \leq S,
$$

ce qui donnera l'inégalité précédente en vertu du lemme 3.18 appliqué avec $c=40$. Étant donné les valeurs de $\varepsilon_{0}$ et $\eta$, le nombre $a$ est égal à

$$
\frac{2 g^{g+1}}{1-0,06}\left(\frac{50+3 g^{-g}}{3}\right)\left(1+\frac{2,11}{g}+\frac{2}{g^{2}}+\frac{0,18}{3+50 g^{g}}\left(\frac{1}{g^{2}}+\frac{1}{g^{3}}\right)\right) \frac{D}{\log E} .
$$

Comme $g \geq 2$, il est donc plus petit que $92 g^{g+1} D / \log E$. En particulier on a

$$
\log a \leq 6 g(\log g) \max \left\{1, \log \frac{D}{\log E}\right\} .
$$

Par ailleurs, dans la formule définissant $\beta$ (lemme 3.26), mettons en facteur la quantité $g^{6} \max \{(\log E) / D, h\}$ et remplaçons $S$ par 1 . Ce qui reste est une fonction décroissante en $g$ qui est donc inférieure à sa valeur en $g=2$. Nous en déduisons $\beta \leq 2 g^{6} \max \{(\log E) / D, h\}$ puis, en minorant $\gamma$ par $g^{2}$, nous obtenons

Nous trouvons ainsi

$$
a b \leq 184 g^{g+5} \max \left\{1, \frac{D h}{\log E}\right\} .
$$

$$
\left(1+\frac{\log 40}{39}\right) \max \{40 a, a b+a \log a\} \leq 254 g^{g+5} \max \left\{1, \frac{D h}{\log E}, \frac{D}{\log E} \log \left(\frac{D}{\log E}\right)\right\} .
$$

Ce majorant est plus petit que $S$ puisque $254 \leq 247 \times 4 \log (2) \leq 247 g^{2} \log g$.

Fin de la démonstration du théorème 3.1. Les lemmes 3.26 et 3.27 entraînent

$$
\frac{1-\eta}{2 D} \varepsilon_{0}(2 g S+1) \log E \leq x(g S+1)^{2}\left(\widehat{h}_{L}(p)+\frac{\pi}{2 D} E^{2}\|u\|_{L, \sigma_{0}}^{2}\right) .
$$

Comme $x \leq\left((2 S+1) / \operatorname{deg}_{L} A\right)^{1 / g}$ par (1), on a en particulier

$$
\left(\operatorname{deg}_{L} A\right)^{1 / g} \log E \leq \frac{2}{(1-\eta) \varepsilon_{0}} \cdot \frac{(g S+1)^{2}}{2 g S+1}(2 S+1)^{1 / g}\left(D \widehat{h}_{L}(p)+\frac{\pi}{2} E^{2}\|u\|_{L, \sigma_{0}}^{2}\right) .
$$

En mettant $S$ en facteur, nous en déduisons le théorème 3.1 car, en minorant $S$ par 100, on voit que l'expression

$$
\frac{2\left(50 g^{g}+3\right) g}{3(1-\eta)} \times \frac{(1+1 /(g S))^{2}(2+1 / S)^{1 / g}}{2+1 /(g S)}\left(248 g^{g+7} \log g\right)^{1+1 / g}
$$

est plus petite que $10^{6} g^{2 g+9}(\log g) \leq c_{0}(g)$. 
3.12. Conclusion dans le cas $g=1$. Dans ce paragraphe, nous démontrons le théorème 3.1 dans le cas où $A$ est une courbe elliptique. La démonstration suit exactement les mêmes étapes que dans le cas $g \geq 2$, mais certaines estimations sont modifiées.

À partir de maintenant et jusqu'à la fin du paragraphe, nous supposons $g=1$ et que la polarisation $L$ est la polarisation principale sur $A$. Ce n'est pas une hypothèse restrictive car si le théorème 3.1 est démontré dans ce cas, il suffit de multiplier par $N=\operatorname{deg}_{L} A$ pour obtenir une majoration de $N$ : si $L_{0}$ est la polarisation canonique on a $N\|u\|_{L_{0}, \sigma_{0}}^{2}=\|u\|_{L, \sigma_{0}}^{2}$ et $N \widehat{h}_{L_{0}}(p)=\widehat{h}_{L}(p)$. En procédant de cette manière, la borne pour $N$ est même un peu meilleure que celle du théorème 3.1 puisqu'il n'y a pas de $\log \operatorname{deg}_{L} A$ dans le maximum. Reprenons alors les notations et résultats des paragraphes 3.1 à 3.10 sans changement. Le $\S 3.11$ est modifié et, en particulier, l'estimation du lemme 3.20 devient plus précise :

Lemme 3.28. Soit $(A, L)$ une courbe elliptique principalement polarisée sur un corps de nombres k. Alors on $a$ :

$$
\frac{1}{[k: \mathbb{Q}]} \sum_{\sigma: k \hookrightarrow \mathbb{C}} r\left(A_{\sigma}, L_{\sigma}\right) \leq 1,8 \max \{1, h(A)\}^{1 / 2} .
$$

Démonstration. Par le lemme matriciel d'Autissier, ici utilisé sous la forme de la proposition 3.2 de [GR2] et en utilisant la convexité de $\xi \mapsto \xi^{2}$, on a

$$
\frac{1}{[k: \mathbb{Q}]} \sum_{\sigma: k \hookrightarrow \mathbb{C}} \frac{1}{\rho\left(A_{\sigma}, L_{\sigma}\right)} \leq \sqrt{6,45} \max \{1, h(A)\}^{1 / 2}
$$

où $\rho\left(A_{\sigma}, L_{\sigma}\right)$ désigne le diamètre d'injectivité de $\left(A_{\sigma}, L_{\sigma}\right)$. Nous relions ce nombre au rayon de recouvrement $r\left(A_{\sigma}, L_{\sigma}\right)$ de la même manière que dans la discussion qui précède le lemme 3.20. Toutefois nous utilisons la forme précisée du théorème 2.2 de [Ba] (donnée dans les deux premières lignes de la démonstration) lorsque le réseau est de rang 2. Nous obtenons ainsi

$$
r\left(A_{\sigma}, L_{\sigma}\right) \leq\left(\sqrt{2} \rho\left(A_{\sigma}, L_{\sigma}\right)\right)^{-1}
$$

puis nous concluons avec $\sqrt{6,45 / 2} \leq 1,8$.

Comme précédemment, nous aurons besoin de minorer la hauteur du jet. On a maintenant :

Proposition 3.29. La hauteur de jet ${ }^{\ell} s(m p)$ est supérieure à

$$
-n S^{2} \widehat{h}_{L}(p)-T \max \left\{0, h(A)-\frac{1}{2} \log \pi\right\} .
$$

Démonstration. On a $h_{\bar{F}}\left(\operatorname{jet}^{\ell} s(m p)\right) \geq-\widehat{\mu}_{\max }(\bar{F})=-\widehat{\mu}_{\max }\left(S^{\ell}\left(\overline{t_{A_{K}}^{\mathrm{v}}}\right)\right)-n m^{2} \widehat{h}_{L}(p)$. Puisque $A$ est une courbe elliptique (principalement polarisée), on a de plus :

$$
\widehat{\mu}_{\max }\left(S^{\ell}\left(\overline{t_{A_{K}}^{v}}\right)\right)=\widehat{\mu}\left(\overline{t_{A_{K}}^{\mathrm{v}} \otimes \ell}\right)=-\ell \widehat{\mu}\left(\overline{t_{A_{K}}}\right)=\ell\left(h(A)-\frac{1}{2} \log \pi\right)
$$

(la formule pour la pente de $\overline{t_{A_{K}}}$ est rappelée page 7 ). L'estimation annoncée découle alors de $\ell \leq T$ et $|m| \leq S$

À l'instar du lemme 3.22, pour démontrer le théorème 3.1, nous pouvons supposer (ce que nous ferons désormais) que

$$
\|u\|_{L, \sigma_{0}}^{2} \leq \frac{2}{\pi e^{2} c_{0}} \quad \text { où } c_{0}=c_{0}(1)=8,3.10^{8}
$$

est la constante du théorème 3.1. Dans la suite, nous utiliserons également $x \leq 2 S+1$ et nous noterons $h=\max \{1, h(A)\}$.

Lemme 3.30. Pour tout nombre réel $E^{\prime} \geq 1$, on a les majorations suivantes : 
(i)

(ii)

$$
\frac{1}{D} \sum_{\sigma: k \hookrightarrow \mathbb{C}} \log ^{+}\left(\pi x r\left(A_{\sigma}, L_{\sigma}\right)+\sqrt{\frac{\pi x}{2}}\right) \leq 2,43+\log S+\frac{1}{2} \log h .
$$

$$
\log ^{+}\left(\pi x E^{\prime}(S+1)\|u\|_{L, \sigma_{0}}+\sqrt{\frac{\pi x}{2}}\right) \leq-9,4+\log E^{\prime}+2 \log S .
$$

Démonstration. Reprenons la démonstration du lemme 3.23 (i) en utilisant le lemme 3.28 au lieu du lemme 3.20. Nous obtenons d'abord :

$$
\frac{1}{D} \sum_{\sigma: k \hookrightarrow \mathbb{C}} \log ^{+}\left(\pi x r\left(A_{\sigma}, L_{\sigma}\right)+\sqrt{\frac{\pi x}{2}}\right) \leq \max \left\{1, \log \left(1,8 \pi x \sqrt{h}+\sqrt{\frac{\pi x}{2}}\right)\right\} .
$$

En utilisant $x \leq 2 S+1$, nous avons alors

$$
1,8 \pi x \sqrt{h}+\sqrt{\frac{\pi x}{2}} \leq\left(1,8 \pi+\sqrt{\frac{\pi}{2(2 S+1)}}\right)\left(2+S^{-1}\right) S \sqrt{h}
$$

et, en minorant $S$ par 2919, ce dernier majorant est lui-même plus petit que $\exp (2,43) S \sqrt{h}$. Pour (ii), la démonstration est similaire à celle du point (ii) du lemme 3.23. Le terme devant $E^{\prime} S^{2}$ que l'on obtient est

$$
\frac{1}{e} \sqrt{\frac{2 \pi}{c_{0}}}\left(1+S^{-1}\right)\left(2+S^{-1}\right)+\frac{\sqrt{3 \pi / 2}}{S^{3 / 2}} .
$$

En y injectant $S \geq 2919$ et la valeur $c_{0}=8,3.10^{8}$ nous avons alors une majoration par $\exp (-9,4)$.

Comme dans le cas $g \geq 2$, la démonstration se scinde maintenant en deux cas, selon qu'on se trouve dans le cas non périodique ou dans le cas périodique.

3.12.1. Conclusion dans le cas non périodique $(g=1)$. Commençons par énoncer l'inégalité clef qui conduira à la minoration de $x$, puis au théorème 3.1 .

Lemme 3.31. Posons

$$
\aleph_{1}=\frac{1-\varepsilon_{0}}{2 D} \log \left(1+\frac{\varepsilon_{0}^{2}}{11,6}\right)+\frac{\varepsilon_{0}}{D} \log \left(\frac{2}{\varepsilon_{0}}\left(1+\frac{3}{2 S_{0}}\right)\right)+\frac{\pi \varepsilon_{0}}{2 D} \quad \text { et } \quad \aleph_{2}=\beta+\gamma \log S,
$$

où

$$
\beta=\frac{1-\varepsilon_{0}}{D} \log E+\frac{1}{2} \log h+h \quad \text { et } \quad \gamma=3+2 \varepsilon_{0} .
$$

Alors on $a$ :

$$
\frac{1-\varepsilon_{0}}{D}\left(2 S_{0}+1\right) \log E \leq x S^{2}\left(\widehat{h}_{L}(p)+\frac{\pi}{2 D} E^{2}\|u\|_{L, \sigma_{0}}^{2}\right)+\left(2 S_{0}+1\right) \aleph_{1}+\aleph_{2} .
$$

Démonstration. Reprenons la démonstration du lemme 3.24, mais en utilisant le lemme 3.30 au lieu du lemme 3.23 ainsi que la proposition 3.29 au lieu de la proposition 3.21 pour minorer la hauteur du jet. Les quatre premières inégalités de la démonstration du lemme 3.24 demeurent quasi-inchangées, à ceci près que 3, 09 doit être maintenant remplacé par 2,43 et $-9,52$ par $-9,4$. Nous obtenons ensuite la même inégalité (7), quitte à y opérer les mêmes remplacements, et quitte à remplacer le terme de la dernière ligne par $T\left(h-\frac{1}{2} \log \pi\right)$, provenant de la minoration de la hauteur du jet. Nous terminons alors comme précédemment : nous divisons l'inégalité par $T$, puis nous faisons tendre $T$ vers $+\infty$ et $\epsilon$ vers 0 . Après avoir utilisé les mêmes majorations finales que dans la preuve du lemme 3.24 , sauf pour $\log \|u\|_{L, \sigma_{0}}$ que nous majorons ici par 0 , nous obtenons l'inégalité annoncée. Notons que seule l'expression de $\beta$ se trouve finalement modifiée.

Il reste encore à estimer $\aleph_{1}$ et $\aleph_{2}$ avant de conclure. 
Lemme 3.32. On $a$ :

$$
\aleph_{1} \leq \frac{1-\varepsilon_{0}}{4 D} \log E \quad \text { et } \quad \aleph_{2} \leq \frac{1-\varepsilon_{0}}{4 D}\left(2 S_{0}+1\right) \log E .
$$

Démonstration. La première inégalité se vérifie sans difficulté numériquement en minorant $S_{0}$ par 50 et en utilisant la valeur explicite $\varepsilon_{0}=0,044$ (qui a été choisie dans ce but). Pour la seconde, nous procédons comme dans la démonstration du lemme 3.25 . On a ici $S_{0} \geq 50$, donc $\frac{2 \widetilde{S}_{0}+1}{2 S_{0}+1} \leq 1+\frac{1}{S_{0}} \leq 1,02$. Ainsi, si l'on pose

$$
\delta=\frac{\varepsilon_{0}\left(1-\varepsilon_{0}\right)}{4,08 D}, \quad a=\frac{\gamma}{\delta \log E} \quad \text { et } \quad b=\frac{\beta}{\gamma},
$$

il suffit à nouveau de vérifier l'inégalité $a(b+\log S) \leq S$ ou bien encore, par le lemme 3.18 appliqué avec $c=7,41$, que

$$
a\left(1+\frac{\log c}{c-1}\right) \max \{c, b+\log a\} \leq S
$$

On a :

d'où l'on déduit

$$
a=\frac{4,08\left(3+2 \varepsilon_{0}\right)}{\varepsilon_{0}\left(1-\varepsilon_{0}\right)} \cdot \frac{D}{\log E} \leq 300 \frac{D}{\log E},
$$

$$
\log a \leq 6,71 \max \left\{1, \log \left(\frac{D}{\log E}\right)\right\} .
$$

D'autre part, puisque $\log h \leq h / e$, on a :

$$
b \leq \frac{1-\varepsilon_{0}}{3+2 \varepsilon_{0}} \cdot \frac{\log E}{D}+\left(\frac{1+(2 e)^{-1}}{3+2 \varepsilon_{0}}\right) h \leq 0,7 \max \left\{h, \frac{\log E}{D}\right\} .
$$

Il résulte de ces estimations :

$$
a b+a \log a \leq 2223 \max \left\{1, \frac{D h}{\log E}, \frac{D}{\log E} \log \left(\frac{D}{\log E}\right)\right\} .
$$

Il ne reste plus qu'à observer que $(1+\log (7,41) / 6,41) \times 2223 \leq 2918$ ce qui entraîne l'inégalité voulue pour $S$ en utilisant $\widetilde{S}-1 \leq S$.

Fin de la démonstration du théorème 3.1. Les lemmes 3.31 et 3.32 et $x \leq 2 S+1$ entraînent

$$
\frac{1-\varepsilon_{0}}{2 D}\left(2 S_{0}+1\right) \log E \leq(2 S+1) S^{2}\left(\widehat{h}_{L}(p)+\frac{\pi}{2 D} E^{2}\|u\|_{L, \sigma_{0}}^{2}\right) .
$$

Par définition, on a $2 \widetilde{S}_{0}+1=\varepsilon_{0}(2 S+1) / 2$ et on a vu un peu plus haut que $2 \widetilde{S}_{0}+1 \leq 1,02\left(2 S_{0}+1\right)$. Nous en déduisons $(2 S+1) /\left(2 S_{0}+1\right) \leq 2,04 / \varepsilon_{0}$ puis

$$
\log E \leq \frac{4,08}{\varepsilon_{0}\left(1-\varepsilon_{0}\right)} S^{2}\left(D \widehat{h}_{L}(p)+\frac{\pi}{2} E^{2}\|u\|_{L, \sigma_{0}}^{2}\right)
$$

qui, compte tenu de la valeur de $\varepsilon_{0}$ et de $S \leq 2919 M$, conduit bien au théorème 3.1 .

3.12.2. Conclusion dans le cas périodique $(g=1)$. A priori ce cas devrait être le plus simple car il signifie qu'il existe un entier $m \in\{1, \ldots, 2 S\}$ tel que $m u$ soit une période de $A_{\sigma_{0}}$ (en particulier $\widehat{h}_{L}(p)=0$ ). Par le lemme matriciel d'Autissier (voir la proposition 3.2 de [GR2]), on a donc

$$
2 S\|u\|_{L, \sigma_{0}} \geq \rho\left(A_{\sigma_{0}}, L_{\sigma_{0}}\right) \geq D^{-1 / 2}(6,45 \max \{1, h(A)\})^{-1 / 2} .
$$

Malheureusement, remise sous la forme du théorème 3.1, cette inégalité s'avère plus faible en la hauteur de $A$ que le théorème 3.1 (dépendance polynomiale au cube plutôt qu'au carré). Nous allons donc suivre la même démarche que dans le cas périodique avec $g \geq 2$. Le pendant du lemme 3.26 est le suivant : 
Lemme 3.33. Posons

$$
\aleph_{1}=\frac{\varepsilon_{0}\left(1-\varepsilon_{0}\right)}{2 D} \log \frac{4}{3}+\frac{\varepsilon_{0}^{2}}{2 D} \pi \quad \text { et } \quad \aleph_{2}=\beta+\gamma \log S,
$$

où

Alors on $a$ :

$$
\beta=\frac{1-\varepsilon_{0}}{D} \log E+\log h+h \quad \text { et } \quad \gamma=4+2 \varepsilon_{0}^{2}
$$

$$
\frac{\left(1-\varepsilon_{0}\right) \varepsilon_{0}}{D}(2 S+1) \log E \leq x(S+1)^{2}\left(\frac{\pi}{2 D} E^{2}\|u\|_{L, \sigma_{0}}^{2}\right)+(2 S+1) \aleph_{1}+\aleph_{2} .
$$

Démonstration. Nous reprenons la démonstration du lemme 3.26, mais en utilisant le lemme 3.30 à la place du lemme 3.23 ainsi que la proposition 3.29 au lieu de la proposition 3.21. Comme $S \geq 2919$, l'inégalité $(g+1) \log S \leq \varepsilon_{0} \pi g S / 2$ de la page 30 reste valable si $g=1$, ce qui permet d'aboutir à nouveau à l'inégalité (8), à ceci près que 3,09 doit être maintenant remplacé par $2,43,-9,52$ par $-9,4$ et la dernière ligne doit être remplacée par $T(h-(\log \pi) / 2)+o(T)$. De plus le terme $\log ^{+}\|u\|_{L, \sigma_{0}}$ est maintenant nul et $\log \left(1 / u_{g}\right)$ doit être majoré au moyen du (i) de la proposition 3.5. Nous terminons exactement comme dans la démonstration du lemme 3.26, en notant que la quantité $2 \log S-9,4$ est bien positive.

Majorons maintenant $\aleph_{1}$ et $\aleph_{2}$ :

Lemme 3.34. On $a$ :

$$
\aleph_{1} \leq \frac{\left(1-\varepsilon_{0}\right) \varepsilon_{0}}{4 D} \log E \quad \text { et } \quad \aleph_{2} \leq \frac{\left(1-\varepsilon_{0}\right) \varepsilon_{0}}{4 D}(2 S+1) \log E .
$$

Démonstration. La première inégalité se vérifie sans difficulté numériquement, en utilisant la valeur explicite $\varepsilon_{0}=0,06$ (choisie pour cela). Vérifions la seconde inégalité. Nous procédons comme dans le lemme 3.27 en posant

$$
\delta=\frac{\varepsilon_{0}\left(1-\varepsilon_{0}\right)}{2 D}, \quad a=\frac{\gamma}{\delta \log E} \quad \text { et } \quad b=\frac{\beta}{\gamma},
$$

et en observant qu'il s'agit de montrer $a(b+\log S) \leq S$. Par le lemme 3.18 (avec $c=2$ ), cette inégalité découle de $1,7 a \max \{2, b+\log a\} \leq S$, que nous allons maintenant montrer. On a

$$
a \leq \frac{2\left(4+2 \varepsilon_{0}^{2}\right)}{\varepsilon_{0}\left(1-\varepsilon_{0}\right)} \cdot \frac{D}{\log E} \leq 143 \frac{D}{\log E} \quad \text { et } \quad \log a \leq 6 \max \left\{1, \log \left(\frac{D}{\log E}\right)\right\} .
$$

Par ailleurs, on a $\beta \leq 3 \max \{(\log E) / D, h\}$ et donc $b \leq \max \{(\log E) / D, h\}$. En réunissant ces estimations, nous avons donc

$$
1,7 \max \{2 a, a b+a \log a\} \leq 1702 \max \left\{1, \frac{D}{\log E}, \frac{D}{\log E} \log \left(\frac{D}{\log E}\right), \frac{D h(A)}{\log E}\right\} .
$$

Cette borne est clairement inférieure à $S$, démontrant ainsi la majoration souhaitée pour $\aleph_{2}$.

Fin de la démonstration du théorème 3.1. Les lemmes 3.33 et 3.34 entraînent

$$
\frac{\varepsilon_{0}\left(1-\varepsilon_{0}\right)}{2 D}(2 S+1) \log E \leq x(S+1)^{2}\left(\frac{\pi}{2 D} E^{2}\|u\|_{L, \sigma_{0}}^{2}\right) .
$$

Ici $x \leq 2 S+1$ et la borne se simplifie en

$$
\log E \leq \frac{2(S+1)^{2}}{\varepsilon_{0}\left(1-\varepsilon_{0}\right)}\left(\frac{\pi}{2} E^{2}\|u\|_{L, \sigma_{0}}^{2}\right) .
$$

Le théorème 3.1 s'obtient alors dans ce cas (périodique et $A$ courbe elliptique) avec la constante $2 \times(2919+1)^{2} /(0,06 \times 0,94) \leq 3,1.10^{8}$. 


\section{DÉmonstrations des théorÈmes de l'introduction}

Dans cette partie nous établissons plusieurs conséquences du théorème 3.1 et nous démontrons les résultats présentés dans l'introduction. Le cadre et les notations sont ceux du début de l'introduction.

4.1. Démonstration du théorème 1.1. Nous allons commencer par établir un résultat un peu plus précis. Soit $k^{\prime}$ une extension finie de $k$ sur laquelle la variété abélienne $A_{u}$ est définie. Posons $D^{\prime}=\left[k^{\prime}: \mathbb{Q}\right]$ et $g_{u}=\operatorname{dim} A_{u}$.

Théorème 4.1. Soit $E \geq e$ un nombre réel. Posons

$$
M^{\prime}=\max \left\{1, \frac{D^{\prime}}{\log E}, \frac{D^{\prime}}{\log E} \log \frac{D^{\prime}}{\log E}, \frac{D^{\prime} h(A)}{\log E}, \frac{D^{\prime}}{\log E} \log \left(\frac{D^{\prime} \widehat{h}_{L}(p)+\frac{\pi}{2} E^{2}\|u\|_{L, \sigma_{0}}^{2}}{\log E}\right)\right\} .
$$

Si $u \neq 0$, on $a$

$$
\left(\operatorname{deg}_{L} A_{u}\right)^{1 / g_{u}} \leq c_{1}\left(g_{u}\right) g^{1+1 / g_{u}}\left(\frac{D^{\prime} \widehat{h}_{L}(p)+\frac{\pi}{2} E^{2}\|u\|_{L, \sigma_{0}}^{2}}{\log E}\right)\left(M^{\prime}\right)^{1+1 / g_{u}}
$$

où $c_{1}(1)=8,3.10^{11}$ et $c_{1}(x)=5.10^{8} x^{2 x+12}(\log x)^{3}$ si $x \geq 2$.

Démonstration. Nous appliquons le théorème 3.1 à $\left(A_{u}, k^{\prime}\right)$ muni d'un plongement quelconque qui prolonge $\sigma_{0}$, théorème dont l'hypothèse principale est naturellement vérifiée par $A_{u}$. La quantité $\left(\operatorname{deg}_{L} A_{u}\right)^{1 / g_{u}} \log E$ est alors plus petite que

$$
c_{0}\left(g_{u}\right)\left(D^{\prime} \widehat{h}_{L}(p)+\frac{\pi}{2} E^{2}\|u\|_{L, \sigma_{0}}^{2}\right) \max \left\{1, \frac{D^{\prime}}{\log E}, \frac{D^{\prime}}{\log E} \log \frac{D^{\prime}}{\log E}, \frac{D^{\prime} h\left(A_{u}\right)}{\log E}, \frac{D^{\prime} \log \operatorname{deg}_{L} A_{u}}{\log E}\right\}^{1+\frac{1}{g_{u}}} .
$$

Si ce dernier maximum est atteint pour l'un des trois premiers termes, le théorème découle simplement de $c_{0}\left(g_{u}\right) \leq c_{1}\left(g_{u}\right)$. Dans la suite nous supposerons donc ce maximum égal à $\left(D^{\prime} / \log E\right) \max \left\{h\left(A_{u}\right), \log \operatorname{deg}_{L} A_{u}\right\}$. Comme $A_{u}$ est une sous-variété abélienne de $A$, sa hauteur de Faltings est majorée en fonction de celle de $A$ :

$$
h\left(A_{u}\right) \leq h(A)+\left(g-g_{u}\right) \log \sqrt{2 \pi}+\log \operatorname{deg}_{L} A_{u}
$$

(voir, par exemple, le paragraphe 2.3 de [GR2]). En remplaçant dans l'inégalité précédente, nous obtenons une majoration de la forme $\xi \leq a(b+\log \xi)$ avec $\xi=\left(\operatorname{deg}_{L} A_{u}\right)^{1 /\left(g_{u}+1\right)}$,

$$
a=\left(g_{u}+1\right) c_{0}\left(g_{u}\right)^{g_{u} /\left(g_{u}+1\right)}\left(\frac{D^{\prime} \widehat{h}_{L}(p)+\frac{\pi}{2} E^{2}\|u\|_{L, \sigma_{0}}^{2}}{\log E}\right)^{g_{u} /\left(g_{u}+1\right)} \frac{D^{\prime}}{\log E}
$$

et

$$
b=\left(g_{u}+1\right)^{-1}\left(1+\left(g-g_{u}\right) \log \sqrt{2 \pi}\right) \max \{1, h(A)\} .
$$

En appliquant le lemme 3.18 nous obtenons $\xi \leq a\left(1+\frac{\log c}{c-1}\right) \max \{c, b+\log a\}$. Avant de choisir $c$, observons que la quantité $b+\log a$ est majorée par

$$
\left(\frac{\left(g-g_{u}\right) \log \sqrt{2 \pi}}{g_{u}+1}+\log \left(g_{u}+1\right)+\frac{g_{u} \log c_{0}\left(g_{u}\right)}{g_{u}+1}+2\right) \frac{M^{\prime} \log E}{D^{\prime}} .
$$

L'expression entre parenthèses s'écrit $g s+t$ avec $s, t$ nombres réels positifs, qui est naturellement plus petit que $g\left(s+t / g_{u}\right)$ puisque $g \geq g_{u}$. De la sorte, on trouve

$$
b+\log a \leq g\left(\frac{\log \left(g_{u}+1\right)}{g_{u}}+\frac{\log c_{0}\left(g_{u}\right)}{g_{u}+1}+\frac{2}{g_{u}}\right) \frac{M^{\prime} \log E}{D^{\prime}} .
$$

En utilisant la valeur exacte de $c_{0}\left(g_{u}\right)$, nous avons $b+\log a \leq 13 g\left(M^{\prime} \log E\right) / D^{\prime}$ si $g_{u}=1$ et $b+\log a \leq 14,1 g\left(\log g_{u}\right)\left(M^{\prime} \log E\right) / D^{\prime}$ si $g_{u} \geq 2$. Choisissons alors $c=13$ si $g_{u}=1$ et $c=19$ si $g_{u} \geq 2$. Ces valeurs sont inférieures aux majorants de $b+\log a$ précédents. Si l'on remplace dans 
la majoration de $\xi$ et que nous élevons le tout à la puissance $\left(g_{u}+1\right) / g_{u}$, nous obtenons l'inégalité souhaitée pour $\left(\operatorname{deg}_{L} A_{u}\right)^{1 / g_{u}}$ avec la constante $4 \times(13(1+(\log 13) / 12))^{2} \times c_{0}(1)$ si $g_{u}=1$ et la constante

$$
c_{0}\left(g_{u}\right)\left(14,1\left(1+\frac{\log 19}{18}\right)\left(g_{u}+1\right) \log g_{u}\right)^{1+1 / g_{u}} \quad \text { si } g_{u} \geq 2 .
$$

Il est alors facile de voir que ces quantités sont plus petites que $c_{1}\left(g_{u}\right)$.

Il reste maintenant à déduire le théorème 1.1 de ce théorème. Pour cela nous utiliserons le résultat suivant, conséquence facile du théorème 1.1 de [Ré2].

Proposition 4.2. Soit $A$ une variété abélienne de dimension $g$ définie sur un corps de nombres $k$. Posons $f(g)=\alpha(g) \times 2.6^{g-1} g$ ! où $\alpha(g)=1$ sauf dans les cas suivants $: \alpha(2)=2, \alpha(4)=5$ et $\alpha(6)=7 / 6$. Alors il existe une extension galoisienne $k_{A}$ telle que toute sous-variété abélienne de $A$ est définie sur $k_{A}$ et de degré relatif $\left[k_{A}: k\right] \leq f(g)$.

Démonstration. Considérons une sous-variété abélienne $B$ de $A$ (définie sur une clôture algébrique $\bar{k}$ de $k$ ) et notons $B^{\prime}$ un quasi-supplémentaire de $B$ dans $A$, c'est-à-dire que $A$ est isogène à $B \times B^{\prime}$ sur $\bar{k}$ (théorème de réductibilité de Poincaré). La composée de l'isogénie avec, à gauche, la projection $B \times B^{\prime} \rightarrow B$ suivie de l'injection $B \hookrightarrow A$ fournit un endomorphisme $\varphi \in \operatorname{End}_{\bar{k}}(A)$ tel que $B=\varphi(A)$. Par définition $\varphi$ est défini sur l'extension galoisienne minimale $k_{A} / k$ telle que $\operatorname{End}_{\bar{k}}(A)=\operatorname{End}_{k_{A}}(A)$. Comme $A$ est aussi définie sur $k_{A}$, il en est de même pour $B=\varphi(A)$. On conclut au moyen du théorème 1.1 de [Ré2] qui affirme $\left[k_{A}: k\right] \leq f(g)$.

Dans la suite, pour certaines parties des calculs faisant intervenir $f(g)$, nous serons amenés à utiliser l'encadrement plus simple $48 \leq f(g) \leq(3,8 g)^{g}$ valide pour $g \geq 2$. Posons alors $E=\max \{e, \sqrt{2 f(g) / \pi}\}$ qui vaut $e$ seulement si $g=1$. Dans le majorant du théorème 4.1, nous remplaçons $D^{\prime} \widehat{h}_{L}(p)+\frac{\pi}{2} E^{2}\|u\|_{L, \sigma_{0}}^{2} \operatorname{par}(\pi / 2) E^{2}\left(D \widehat{h}_{L}(p)+\|u\|_{L, \sigma_{0}}^{2}\right)\left(\right.$ car $D^{\prime} \leq f(g) D$ par la proposition 4.2) et, au moyen de la relation $h(A)=h_{F}(A)+(g / 2) \log \pi$, le maximum $M^{\prime}$ par

$$
\frac{f(g)}{\log E}\left(1+\log \max \left\{\pi^{g / 2}, \frac{\pi E^{2}}{2 \log E}\right\}\right) \times D \max \left\{1, \log D, h_{F}(A), \log \left(D \widehat{h}_{L}(p)+\|u\|_{L, \sigma_{0}}^{2}\right)\right\} .
$$

Ainsi le théorème 1.1 est établi avec la constante

$$
c_{2}=c_{1}\left(g_{u}\right) g^{1+1 / g_{u}} \times \frac{\pi E^{2}}{2 \log E} \times\left(\frac{f(g)}{\log E}\left(1+\log \max \left\{\pi^{g / 2}, \frac{\pi E^{2}}{2 \log E}\right\}\right)\right)^{1+1 / g_{u}}
$$

que nous allons maintenant simplifier pour aboutir à la constante du théorème 1.1. Commençons par observer que $\pi E^{2} /(2 \log E) \geq \pi^{g / 2}$ est toujours vrai. En effet si $g=1$ alors $E=e$ et $\pi E^{2} /(2 \log E)=\pi e^{2} / 2 \geq \sqrt{\pi}$. Si $g \geq 2$ nous minorons $E^{2}$ par $e^{g-1}$ et, par croissance de $x \mapsto x / \log x$ pour $x \geq e$, nous avons $\pi E^{2} /(2 \log E) \geq \pi e^{g-1} /(g-1) \geq \pi^{g / 2}$. Ainsi on a

$$
c_{2}=c_{1}\left(g_{u}\right) g^{1+1 / g_{u}} \times \frac{\pi E^{2}}{2 \log E} \times\left(\frac{f(g)}{\log E}\left(\log \frac{e \pi E^{2}}{2 \log E}\right)\right)^{1+1 / g_{u}} .
$$

Si $g=1$ alors $g_{u}=1$ et $A_{u}=A$ si bien que $k^{\prime}=k$ et l'on peut remplacer $f(1)$ par 1 dans $c_{2}$. On a alors $c_{2}=8,3.10^{11} \times\left(\pi e^{2} / 2\right) \times\left(\log \left(\pi e^{3} / 2\right)\right)^{2} \leq 1,2.10^{14}$. Si $g_{u}=1<g$ alors $E \geq \sqrt{96 / \pi} \geq 5,5$ donc

$$
\log \frac{e \pi E^{2}}{2 \log E} \leq 2,6 \log E
$$

puis $c_{2} \leq 8,3.10^{11}(2,6)^{2} g^{2} f(g)^{3} / \log E$. En majorant $f(g)$ par $(3,8 g)^{g}$ et en utilisant $8,3 \times$ $(2,6)^{2} g^{2} /(\log \sqrt{2 / \pi}+(g / 2) \log (3,8 g)) \leq 63 g$, on trouve $c_{2} \leq 6,3.10^{12} g(3,8 g)^{3 g} \leq(100 g)^{4 g}$. Supposons maintenant $g_{u} \geq 2$. Comme précédemment, on a

$$
c_{2} \leq c_{1}\left(g_{u}\right)\left(\frac{\pi E^{2}}{2 \log E}\right)(2,6 g f(g))^{1+1 / g_{u}} .
$$


En remplaçant $E$ par $\sqrt{2 / \pi}(3,8 g)^{g / 2}, f(g)$ par $(3,8 g)^{g}$ et $c_{1}\left(g_{u}\right)$ par sa valeur nous obtenons

$$
c_{2} \leq 1,3.10^{9}\left(\frac{(3,8 g)^{2 g} g^{13}(\log g)^{3}}{\log \sqrt{2 / \pi}+(g / 2) \log (3,8 g)}\right) g_{u}^{2 g_{u}}\left(2,6 g(3,8 g)^{g}\right)^{1 / g_{u}} .
$$

Pour $a>1$, l'application $x \in\left[1,+\infty\left[\mapsto x^{2 x} a^{1 / x}\right.\right.$ est convexe et donc $\sup \left\{x^{2 x} a^{1 / x} ; 2 \leq x \leq g\right\}=$ $\max \left\{16 \sqrt{a}, g^{2 g} a^{1 / g}\right\}$. En appliquant ce principe avec $a=2,6 g(3,8 g)^{g}$ et $x=g_{u}$, on constate que le maximum est atteint pour $g_{u}=g$. Il vient

$$
c_{2} \leq 1,3.10^{9} g^{4 g}\left(\frac{(3,8)^{2 g+1} g^{14}(\log g)^{3}(2,6 g)^{1 / g}}{\log \sqrt{2 / \pi}+(g / 2) \log (3,8 g)}\right)
$$

puis $c_{2} \leq(96 g)^{4 g}$.

4.2. Démonstration du théorème 1.2. Comme précédemment notons $D^{\prime}$ le degré minimal d'un corps de définition de la variété abélienne $A_{u}$. Soit $E>0$ le nombre réel défini par

$$
\frac{\pi}{2} E^{2}\|u\|_{L, \sigma_{0}}^{2}=D^{\prime} \max \left\{1, h_{F}(A), \widehat{h}_{L}(p)\right\} .
$$

En particulier l'inégalité $\log \|u\|_{L, \sigma_{0}} \geq-\log \sqrt{\pi / 2}-\log E$ est toujours vérifiée. Dès $\operatorname{lors,~si~} \log E \leq$ $D^{\prime} \max \left\{1, h(A), \widehat{h}_{L}(p)\right\}$, le théorème 1.2 est immédiat en utilisant $D^{\prime} \leq(3,8 g)^{g} D$ et la relation $h(A)=h_{F}(A)+(g / 2) \log \pi$. Supposons maintenant $\log E \geq D^{\prime} \max \left\{1, h(A), \widehat{h}_{L}(p)\right\}$, inégalité qui implique $E \geq e$ et $\log E \geq(\pi / 2) E^{2}\|u\|_{L, \sigma_{0}}^{2}$. Nous utilisons alors le théorème 4.1 avec $E$ et nous minorons le degré de $A_{u}$ par 1 . Le maximum qui apparaît dans le majorant du théorème vaut 1 par hypothèse sur $\log E$. Nous obtenons ainsi

$$
\log E \leq c_{1}\left(g_{u}\right) g^{1+1 / g_{u}} D^{\prime}\left(\widehat{h}_{L}(p)+\max \left\{1, h_{F}(A), \widehat{h}_{L}(p)\right\}\right)
$$

puis le théorème 1.2 car $\log \sqrt{\frac{\pi}{2}}+2 c_{1}\left(g_{u}\right) g^{1+1 / g_{u}}(3,8 g)^{g}$ est plus petit que $(196 g)^{3 g}$ si $g \geq 2$ et $6,4.10^{12}$ si $g=1$, constantes elles-mêmes plus petites que $(196 g)^{3 g+3}$.

4.3. Démonstration du théorème 1.3. Comme nous l'avons mentionné dans l'introduction, le théorème 1.3 est une conséquence du théorème des périodes généralisé et du premier théorème de Minkowski, théorème qui revêt ici la forme suivante. Nous ne faisons aucune hypothèse sur le point $p \in A(k)$.

Lemme 4.3. Pour tous nombres réels a,b strictement positifs, il existe $(\ell, \omega) \in\left(\mathbb{Z} \times \Omega_{A_{\sigma_{0}}}\right) \backslash\{0\}$ tel que

$$
a \widehat{h}_{L}(\ell p)+b\|\ell u+\omega\|_{L, \sigma_{0}}^{2} \leq(g+1)\left(\widehat{h}_{L}(p) h^{0}(A, L)^{2} a b^{2 g}\right)^{1 /(2 g+1)} .
$$

Démonstration. Si $p$ est un point de torsion alors $\widehat{h}_{L}(p)=0$ et il existe $(\ell, \omega) \in\left(\mathbb{Z} \times \Omega_{A_{\sigma_{0}}}\right) \backslash\{0\}$ tel que $\ell u+\omega=0$, si bien que le membre de gauche de l'inégalité est nul. Le lemme est donc immédiat dans ce cas. Si $p$ n'est pas un point de torsion, la formule

$$
\forall x \in \mathbb{R}, \quad \forall y \in t_{A_{\sigma_{0}}}, \quad\|(x, y)\|^{2}:=a x^{2} \widehat{h}_{L}(p)+b\|x u+y\|_{L, \sigma_{0}}^{2}
$$

définit une métrique euclidienne sur le $\mathbb{R}$-espace vectoriel $\mathbb{R} \times t_{A_{\sigma_{0}}}$ (de dimension $2 g+1$ ). Dans cet espace, le $\mathbb{Z}$-module $\Omega^{\prime}:=\mathbb{Z} \times \Omega_{A_{\sigma_{0}}}$ est un réseau complet. Soit $\omega_{1}, \ldots, \omega_{2 g}$ une $\mathbb{Z}$-base de $\Omega_{A_{\sigma_{0}}}$ de sorte que $\left\{(1,0),\left(0, \omega_{1}\right), \ldots,\left(0, \omega_{2 g}\right)\right\}$ est une base de $\Omega^{\prime}$. Si l'on note $\langle\cdot, \cdot\rangle$ le produit scalaire associé à la norme $\|\cdot\|$, le déterminant de $\Omega^{\prime}$ est celui de la matrice des produits scalaires des vecteurs de cette base. Or, pour tous $1 \leq i, j \leq 2 g$, on a $\langle(1,0),(1,0)\rangle=a \widehat{h}_{L}(p)+b\|u\|_{L, \sigma_{0}}^{2}$, $\left\langle(1,0),\left(0, \omega_{i}\right)\right\rangle=b\left\langle u, \omega_{i}\right\rangle_{L, \sigma_{0}}$ et $\left\langle\left(0, \omega_{i}\right),\left(0, \omega_{j}\right)\right\rangle=b\left\langle\omega_{i}, \omega_{j}\right\rangle_{L, \sigma_{0}}$, où $\langle\cdot, \cdot\rangle_{L, \sigma_{0}}$ est le produit scalaire sur $t_{A_{\sigma_{0}}}$ associé à la norme $\|\cdot\|_{L, \sigma_{0}}$. En écrivant $u$ comme combinaison linéaire réelle des $\omega_{i}$, les propriétés standards du déterminant conduisent à l'égalité

$$
\operatorname{det} \Omega^{\prime}=a \widehat{h}_{L}(p) \times b^{2 g} \operatorname{det}\left(\left\langle\omega_{i}, \omega_{j}\right\rangle_{L, \sigma_{0}}\right)_{1 \leq i, j \leq 2 g} .
$$


Ce dernier déterminant vaut $h^{0}(A, L)^{2}$ (voir, par exemple, la proposition 3 de [BP]). Par définition de la constante d'Hermite $\gamma_{2 g+1}$ en dimension $2 g+1$, il existe $(\ell, \omega) \in\left(\mathbb{Z} \times \Omega_{A_{\sigma_{0}}}\right) \backslash\{0\}$ tel que $\|(\ell, \omega)\|^{2} \leq \gamma_{2 g+1}\left(\operatorname{det} \Omega^{\prime}\right)^{1 /(2 g+1)}$. On conclut avec la borne $\gamma_{2 g+1} \leq g+1$ (voir [MH, p. 17]).

Proposition 4.4. Soit $p \in A(k)$ un point rationnel qui n'est pas de torsion. Si $g=1$ on a

$$
\widehat{h}_{L}(p)^{-1} \leq 10^{40} D^{7} \max \left\{1, \log D, h_{F}(A)\right\}^{6} .
$$

Si $g \geq 2$ on $a$

$$
\widehat{h}_{L}(p)^{-1} \leq(736 g)^{8 g^{2}} D^{4 g+3} h^{0}(A, L)^{2} \max \left\{1, \log D, h_{F}(A)\right\}^{4 g+2} .
$$

L'on sait depuis les travaux de Masser [Ma1, Ma2] que l'on a des majorations du type

$$
\widehat{h}_{L}(p)^{-1} \leq c_{L}(A) D^{2 g+1}(\log (D+1))^{2 g} \quad \text { et } \quad \widehat{h}_{L}(p)^{-1} \leq c_{L}(k, g) \max \left\{1, h_{F}(A)\right\}^{2 g+1}
$$

avec des constantes $c_{L}(A)$ et $c_{L}(k, g)$ qui sont fonction seulement de $A, L$ et $k, g, L$ respectivement (la deuxième majoration n'est démontrée que pour une famille de variétés). Les dépendances en le degré $D$ et en la hauteur de Faltings de $A$ sont donc un peu meilleures que celles de la proposition. Celle-ci a néanmoins l'avantage de donner une borne entièrement explicite et simultanée en les deux paramètres $D$ et $h_{F}(A)$, ce qui, à ce niveau de généralité, est inédit. Citons toutefois l'article de Winckler [Wi] qui donne une majoration fine et explicite de $\widehat{h}_{L}(p)^{-1}$ lorsque $A$ est une courbe elliptique à multiplications complexes.

Démonstration. Commençons par $g \geq 2$. Soit $(\ell, \omega)$ un couple donné par le lemme précédent appliqué avec $a=(3,8 g)^{g} D$ et $b=\pi e^{2} / 2$. Si sa norme (au sens de la démonstration du lemme) est plus grande que 1 alors

$$
\widehat{h}_{L}(p)^{-1} \leq(g+1)^{2 g+1}\left((3,8 g)\left(\frac{\pi e^{2}}{2}\right)^{2}\right)^{g} D h^{0}(A, L)^{2}
$$

et la proposition est démontrée. Sinon, comme $\ell u+\omega \neq 0$ puisque $p$ n'est pas un point de torsion, nous pouvons utiliser le théorème 4.1 avec le logarithme $\ell u+\omega$ de $\ell p$ et $E=e$. Dans l'inégalité donnée par ce théorème, nous majorons $D^{\prime}$ par $(3,8 g)^{g} D$ et nous minorons le degré et la dimension de $A_{\ell u+\omega}$ par 1 . De plus, par hypothèse, le logarithme de $\|(\ell, \omega)\|^{2}$ est négatif, si bien qu'il disparaît du maximum. Nous avons ainsi

$$
1 \leq c_{1}\left(g_{\ell u+\omega}\right) g^{2}\|(\ell, \omega)\|^{2}(3,8 g)^{2 g} D^{2} \max \{1, g \log (3,8 g)+\log D, h(A)\}^{2} .
$$

Comme $h(A)=h_{F}(A)+g \log \sqrt{\pi}$, le maximum dans cette inégalité est plus petit que $(g \log (3,8 g)+$ 1) $\max \left\{1, \log D, h_{F}(A)\right\}$. Si nous remplaçons aussi $\|(\ell, \omega)\|^{2}$ par le majorant donné par le lemme, l'inégalité qui en résulte est celle de la proposition 4.4 mais avec la constante

$$
c(g)=\left(c_{1}\left(g_{\ell u+\omega}\right) g^{2}(g+1)\right)^{2 g+1}(3,8 g)^{g(4 g+3)}\left(\frac{\pi e^{2}}{2}\right)^{2 g}(g \log (3,8 g)+1)^{2(2 g+1)} .
$$

Nous majorons $c_{1}\left(g_{\ell u+\omega}\right)$ par $c_{1}(g)$ (car $c_{1}$ est croissante), $g+1$ par $3 g / 2$ et $g \log (3,8 g)+1$ par $3,7 g \log g$. En appelant $c^{\prime}(g)$ la nouvelle constante ainsi obtenue déduite de $c(g)$, nous voyons que la quantité $\left(\log c^{\prime}(g)-8 g^{2} \log g\right) / g^{2}$ est une combinaison linéaire à coefficients positifs des quantités $1,1 / g, 1 / g^{2}, \log g / g, \log g / g^{2}, \log \log g / g, \log \log g / g^{2}$, qui sont toutes des fonctions décroissantes de $g$ pour $g \geq 6$. Un calcul numérique pour $g \in\{2,3,4,5,6\}$ montre que cette quantité est majorée par sa valeur en $g=2$, soit 52,8 . Dans ce cas, on conclut en observant $\exp (52,8 / 8) \leq 736$. Le cas $g=1$ se traite de manière similaire mais l'on peut économiser le 3,8 puisque $\left(A_{\ell u+\omega}, k^{\prime}\right)=(A, k)$ et donc $D^{\prime}=D$. Nous choisissons donc $a=D$ et la constante $c(g)$ est remplacée par

$$
\left(2 c_{1}(1)\right)^{3}\left(\frac{\pi e^{2}}{2}\right)^{2}(\log \sqrt{\pi}+1)^{6} \leq 10^{40} .
$$

À ce stade nous avons obtenu la borne souhaitée pour $\widehat{h}_{L}(p)^{-1}$ mais seulement lorsque la polarisation est principale $\left(h^{0}(A, L)=1\right)$. Or, lorsque $g=1$, la variété abélienne est une courbe 
elliptique et $L$ est une puissance entière de la polarisation canonique principale $L_{0}$ sur $A$. On a ainsi $\widehat{h}_{L}(p)^{-1} \leq \widehat{h}_{L_{0}}(p)^{-1}$ puis la borne voulue en toute généralité.

Pour obtenir le théorème 1.3, il reste à faire disparaître le degré de $A$ dans le majorant de $\widehat{h}_{L}(p)^{-1}$ donné par cette proposition. Posons $m=\max \left\{D+g^{g}, h_{F}(A)\right\}$ et notons que $\max \left\{1, \log D, h_{F}(A)\right\} \leq m$. Ainsi, lorsque $g=1$, la proposition donne $\widehat{h}_{L}(p)^{-1} \leq 10^{40} m^{13}$ et le théorème 1.3 découle de $10^{40} \leq 2^{133} \leq m^{133}$. Supposons maintenant $g \geq 2$. Nous allons tirer parti de l'astuce de Zarhin. En effet, le théorème 1.3 que l'on cherche à montrer ne dépend pas de $L$ comme faisceau inversible ample et symétrique mais seulement de la polarisation qu'il induit. Étant donné $(A, L)$, on sait qu'il existe une polarisation principale $L^{\prime}$ sur $Z(A)=(A \times \widehat{A})^{4}$ (où $\widehat{A}$ désigne la variété abélienne duale) telle que si $\iota: A \hookrightarrow Z(A)$ désigne l'injection sur le premier facteur de $Z(A)$ alors $\iota^{*} L^{\prime} \simeq L$ (voir le lemme 4.6 de [Ré1]). En particulier la hauteur de NéronTate de $p$ relative à $L$ est égale à celle de $\iota(p)$ relative à $L^{\prime}$. Appliquons alors la proposition 4.4 à $\left(\iota(p), Z(A), L^{\prime}\right)$. On a $h_{F}(Z(A))=8 h_{F}(A)$ et $\operatorname{dim} Z(A)=8 g$. Nous avons donc

$$
\widehat{h}_{L}(p)^{-1} \leq(736 \times 8 g)^{8(8 g)^{2}} D^{32 g+3} \max \left\{1, \log D, 8 h_{F}(A)\right\}^{32 g+2} \leq(5888 g)^{512 g^{2}} 8^{32 g+2} m^{64 g+5} .
$$

Comme $g \geq 2$, on a

$$
(5888 g)^{512 g^{2}} 8^{32 g+2} \leq\left(1+g^{g}\right)^{6007 g} \leq m^{6007 g} .
$$

Le théorème 1.3 découle alors de $6007 g+64 g+5 \leq 6074 g$.

4.4. Démonstration du théorème 1.4. Nous allons commencer par le cas particulier où $A_{p}$ est connexe, pour lequel l'estimation du degré est plus précise. Étant donné une variété abélienne A de dimension g sur $k$, notons

$$
\kappa(\mathrm{A})=\left((14 \mathrm{~g})^{64 \mathrm{~g}^{2}}[k: \mathbb{Q}] \max \left\{1, \log [k: \mathbb{Q}], h_{F}(\mathrm{~A})\right\}^{2}\right)^{1024 \mathrm{~g}^{3}} .
$$

Proposition 4.5. Soit $p \in A(k)$ tel que $A_{p}$ soit une sous-variété abélienne non nulle de $A$ (en particulier $p$ n'est pas un point de torsion). Alors on a

$$
\left(\operatorname{deg}_{L} A_{p}\right)^{1 / \operatorname{dim} A_{p}} \leq \widehat{h}_{L}(p) \kappa\left(A_{p}\right)^{6-1 / \operatorname{dim} A_{p}} .
$$

Démonstration. Nous suivons la démarche de Bertrand expliquée pages 238 et 239 de l'article [Be], démarche dans laquelle se greffent des estimations extraites de l'article [Ré1] de Rémond et la minoration de la hauteur de Néron-Tate de la proposition 4.4.

Par le théorème 1.1 de [GR3], il existe une polarisation $\lambda$ sur $A_{p}$ telle que $\operatorname{deg}_{\lambda} A_{p} \leq \kappa\left(A_{p}\right)$. Notons $A_{1}, \ldots, A_{r}$ les composantes isotypiques de $A_{p}$ définies sur $k$ : chaque $A_{i}$ est une sous-variété abélienne de $A_{p}$, maximale pour l'inclusion et $k$-isogène à une puissance d'une variété abélienne simple. Pour tout $i \in\{1, \ldots, r\}$, notons $g_{i}=\operatorname{dim} A_{i}, d_{i}$ la dimension sur $\mathbb{Q}$ de l'algèbre simple $\operatorname{End}_{k}\left(A_{i}\right) \otimes_{\mathbb{Z}} \mathbb{Q}$ et $\Delta_{i}$ la valeur absolue du discriminant de $\operatorname{End}_{k}\left(A_{i}\right)$. Posons

$$
R_{i}=4^{g_{i}}\left(\frac{\left(d_{i} / 2\right) !}{\pi^{d_{i} / 2}}\right)^{2 g_{i} / d_{i}}\left(\min _{\substack{x \in A_{i}(k) \\ x \text { non de torsion }}} \widehat{h}_{\lambda}(x)\right)^{-g_{i}}\left(\operatorname{deg}_{\lambda} A_{i}\right) \Delta_{i}^{g_{i} / d_{i}} .
$$

L'application somme $s: \prod_{i=1}^{r} A_{i} \rightarrow A_{p}$ est une isogénie de degré minimal et $\prod_{i=1}^{r} h^{0}\left(A_{i}, \lambda\right)=$ $(\operatorname{deg} s) h^{0}\left(A_{p}, \lambda\right)$ (voir [Ré1, Proposition 3.4]). En notant $g_{p}=\operatorname{dim} A_{p}$, Bertrand a montré que

$$
\operatorname{deg}_{L} A_{p} \leq(\operatorname{deg} s)^{2 g_{p}-1}\left(\prod_{i=1}^{r} R_{i}\right) \widehat{h}_{L}(p)^{g_{p}}
$$

qui découle de l'égalité (8) page 238 et du haut de la page 239 de [Be]. Nous avons mis ici une puissance $2 g_{p}$-ème supplémentaire à $\operatorname{deg} s$ pour assurer que les points $P_{i}$ de l'article de Bertrand appartiennent à $A_{i}(k)$ et non seulement au tensorisé par $\mathbb{Q}$. En effet, nous les définissons comme composantes de $\psi(P)$ où $\psi: A_{p} \rightarrow \prod_{i=1}^{r} A_{i}$ est une isogénie telle que $s \circ \psi$ soit la multiplication 
par deg $s$. Au haut de la page 239 de l'article de Bertrand, nous remplaçons alors $P$ par $(\operatorname{deg} s) P=$ $s(\psi(P))$, ce qui fait ressortir un $(\operatorname{deg} s)^{2 g_{p}}$.

Notre tâche est donc de majorer $(\operatorname{deg} s)^{2 g_{p}-1} \prod_{i=1}^{r} R_{i}$. La proposition 2.12 de [Ré1] fournit l'estimation $\Delta_{i}^{g_{i} / d_{i}} \leq \kappa\left(A_{i}\right)$. De plus, comme $d_{i} \leq 2 g_{i}^{2}$ et $\left(d_{i} / 2\right) ! \leq \max \left\{1, d_{i} / 2\right\}^{d_{i} / 2}$, on a

$$
4^{g_{i}}\left(\frac{\left(d_{i} / 2\right) !}{\pi^{d_{i} / 2}}\right)^{2 g_{i} / d_{i}} \leq\left(\frac{2 g_{i}}{\sqrt{\pi}}\right)^{2 g_{i}} .
$$

Enfin grâce à la proposition 4.4 et à l'égalité $\operatorname{deg}_{\lambda} A_{i}=g_{i} ! h^{0}\left(A_{i}, \lambda\right)$, la quantité

$$
\left(\min _{\substack{x \in A_{i}(k) \\ x \text { non de torsion }}} \widehat{h}_{\lambda}(x)\right)^{-g_{i}}\left(\operatorname{deg}_{\lambda} A_{i}\right)
$$

est plus petite que

$$
g_{i} !\left(\left(10 g_{i}\right)^{40 g_{i}^{2}} D^{4 g_{i}+3} \max \left\{1, \log D, h_{F}\left(A_{i}\right)\right\}^{4 g_{i}+2}\right)^{g_{i}} h^{0}\left(A_{i}, \lambda\right)^{2 g_{i}+1},
$$

elle-même inférieure à $\left(\sqrt{\pi} /\left(2 g_{i}\right)\right)^{2 g_{i}} \kappa\left(A_{i}\right) h^{0}\left(A_{i}, \lambda\right)^{2 g_{i}+1}$. En utilisant $h^{0}\left(A_{p}, \lambda\right) \leq \kappa\left(A_{p}\right)$ (choix de $\lambda)$, nous obtenons ainsi

$$
\prod_{i=1}^{r} R_{i} \leq\left((\operatorname{deg} s) \kappa\left(A_{p}\right)\right)^{2 \max _{i}\left\{g_{i}\right\}+1} \prod_{i=1}^{r} \kappa\left(A_{i}\right)^{2} .
$$

Reportons dans ce majorant l'estimation $\kappa\left(A_{i}\right) \leq \kappa\left(A_{p}\right)^{\left(g_{i} / g_{p}\right)^{3}}$ donnée par le lemme 2.8 de [Ré1]. Si $r=1$ alors $A_{1}=A_{p}$ et $s$ est l'identité donc $\operatorname{deg} s=1$. Dans ce cas nous avons

$$
\left(\operatorname{deg}_{L} A_{p}\right) \widehat{h}_{L}(p)^{-g_{p}} \leq \kappa\left(A_{p}\right)^{2 g_{p}+3} \leq \kappa\left(A_{p}\right)^{6 g_{p}-1}
$$

et la proposition est démontrée. Si $r \geq 2$ alors $\max _{i}\left\{g_{i}\right\} \leq g_{p}-1$. De plus, par minimalité de $s$ et en vertu du théorème 1.4 de [GR3], nous avons $\operatorname{deg} s \leq \kappa\left(A_{p}\right)$. Nous obtenons alors

$$
(\operatorname{deg} s)^{2 g_{p}-1} \prod_{i=1}^{r} R_{i} \leq \kappa\left(A_{p}\right)^{a} \quad \text { avec } \quad a=6 g_{p}-3+2 \sum_{i=1}^{r}\left(\frac{g_{i}}{g_{p}}\right)^{3} .
$$

La fonction $\xi \mapsto \xi^{3}$ étant sur-additive, nous avons

$$
\sum_{i=1}^{r}\left(\frac{g_{i}}{g_{p}}\right)^{3} \leq\left(\sum_{i=1}^{r} \frac{g_{i}}{g_{p}}\right)^{3}=1
$$

puis $a \leq 6 g_{p}-1$. Ainsi, dans tous les cas, on a $\left(\operatorname{deg}_{L} A_{p}\right) \leq \kappa\left(A_{p}\right)^{6 g_{p}-1} \widehat{h}_{L}(p)^{g_{p}}$.

De cette proposition, l'on déduit en particulier la borne $\left(\operatorname{deg}_{L} A_{p}\right)^{1 / \operatorname{dim} A_{p}} \leq \widehat{h}_{L}(p) \kappa(A)^{6}$ (car $\kappa\left(A_{p}\right) \leq \kappa(A)$ ) lorsque $A_{p}$ est connexe. Nous allons maintenant supprimer cette dernière hypothèse et remplacer l'exposant 6 par 8 , ce qui fournira la démonstration du théorème 1.4. Considérons un quasi-supplémentaire $B$ (sous-variété abélienne définie sur $k$ ) de la composante neutre $A_{p}^{0}$ de $A_{p}$ dans $A$ donné par le théorème 1.3 de [GR3] : $A=A_{p}^{0}+B$ et $N:=\operatorname{Card} A_{p}^{0} \cap B \leq \kappa(A)$. L'isogénie somme $\varphi: A_{p}^{0} \times B \rightarrow A$ possède un quasi-inverse $\psi: A \rightarrow A_{p}^{0} \times B$ qui est une isogénie vérifiant $\varphi \circ \psi=[N]$. L'image de $p$ par $\psi$ s'écrit $\left(p^{\prime}, q\right)$ avec $p^{\prime} \in A_{p}^{0}(k)$ et $q \in B(k)$. Comme $N p=p^{\prime}+q$, on a $q \in A_{p}(k)$ donc un multiple entier non nul de $q$ appartient au groupe fini $A_{p}^{0} \cap B$ et $q$ est un point de torsion. En notant $e_{B}$ l'exposant du groupe fini $B(k)_{\text {tors }}$, nous avons ainsi $e_{B} p^{\prime}=$ $e_{B} N p$. Cette formule donne $e_{B} N p \in A_{p^{\prime}}$ donc $A_{p} \subset\left[e_{B} N\right]^{-1} A_{p^{\prime}}$ puis, en prenant les composantes neutres, $A_{p}^{0} \subset A_{p^{\prime}}^{0} \subset A_{p^{\prime}}$. Comme $A_{p^{\prime}} \subset A_{p}^{0}$ puisque $p^{\prime} \in A_{p}^{0}$, nous en déduisons $A_{p^{\prime}}=A_{p}^{0}$. Ceci prouve que $A_{p^{\prime}}$ est une variété abélienne (connexe) à laquelle nous pouvons appliquer la borne de la proposition 4.5. L'égalité $e_{B} p^{\prime}=e_{B} N p$ implique également que la hauteur de $p^{\prime}$ est égale 
à $N^{2} \widehat{h}_{L}(p)$. De plus, elle montre que l'ordre $n$ de $p$ dans le groupe (fini) $A_{p} / A_{p}^{0}$ divise $e_{B} N$ et il vérifie donc

$$
n \leq \kappa(A)^{(35 / 16)(\operatorname{dim} B / \operatorname{dim} A)^{3}+1}
$$

car $e_{B} \leq \kappa(B)^{35 / 16}$ [Ré1, Proposition 2.9] et $\kappa(B) \leq \kappa(A)^{(\operatorname{dim} B / \operatorname{dim} A)^{3}}$ [Ré1, Lemme 2.8]. Enfin, l'égalité $A_{p} / A_{p}^{0}=\left\{i p+A_{p^{\prime}} ; 0 \leq i \leq n-1\right\}$ conduit à $\operatorname{deg} A_{p}=n \operatorname{deg} A_{p^{\prime}}$. Le report de ces estimations dans la borne pour $\operatorname{deg} A_{p^{\prime}}$ donnée par la proposition 4.5 fournit avec le lemme 2.8 de [Ré1] :

$$
\left(\operatorname{deg} A_{p}\right)^{1 / \operatorname{dim} A_{p}} \leq n^{1 / \operatorname{dim} A_{p}}\left(N^{2} \widehat{h}_{L}(p)\right) \kappa\left(A_{p^{\prime}}\right)^{6-1 / \operatorname{dim} A_{p}} \leq \widehat{h}_{L}(p) \kappa(A)^{b}
$$

où

$$
b=\frac{35}{16 \operatorname{dim} A_{p}}\left(\frac{\operatorname{dim} B}{\operatorname{dim} A}\right)^{3}+\frac{1}{\operatorname{dim} A_{p}}+2+\left(6-\frac{1}{\operatorname{dim} A_{p}}\right)\left(\frac{\operatorname{dim} A_{p}}{\operatorname{dim} A}\right)^{3} .
$$

Cet exposant $b$ est plus petit que 8 comme on le voit en utilisant $\lambda x^{3}+\mu y^{3} \leq \max \{\lambda, \mu\}(x+y)^{3}$ pour $\lambda, \mu, x, y$ nombres réels positifs et $\operatorname{dim} A=\operatorname{dim} B+\operatorname{dim} A_{p}$. L'exposant 8192 du théorème 1.4 résulte alors de $1024 \times 8$.

\section{RÉFÉRENCES}

[Au] P. Autissier. Un lemme matriciel effectif. Math. Z., 273 (2013), 355-361.

[BW] A. Baker et G. Wüstholz. Logarithmic forms and group varieties. J. reine angew. Math, 442 (1993), $19-62$.

[Ba] W. Banaszczyk. New bounds in some transference theorems in the geometry of numbers. Math. Ann. 296 (1993), 625-635.

[Be] D. Bertrand. Minimal heights and polarizations on group varieties. Duke Math. J., 80 (1995), 223-250.

[BP] D. Bertrand et P. Philippon. Sous-groupes algébriques de groupes algébriques commutatifs. Illinois J. Math, 32 (1988), 263-280.

[Bo1] J.-B. Bost. Périodes et isogénies des variétés abéliennes sur les corps de nombres (d'après D. Masser et G. Wüstholz). Séminaire Bourbaki, volume 237 d'Astérisque. Société Mathématique de France (1996), $115-161$.

[Bo2] J.-B. Bost. Intrinsic heights of stable varieties and abelian varieties. Duke Math. J., 82 (1996), 21-70.

[CW] P. Cijsouw et M. Waldschmidt. Linear forms and simultaneous approximations. Compos. Math., 34 (1977), 173-197.

[Da] S. David. Approximation diophantienne sur les variétés abéliennes. École doctorale de Géométrie diophantienne. Rennes (France), 15-26 juin 2009.

[Ga1] É. Gaudron. Formes linéaires de logarithmes effectives sur les variétés abéliennes. Ann. Sci. École Norm. Sup., 39 (2006), 699-773.

[Ga2] É. Gaudron. Pentes des fibrés vectoriels adéliques sur un corps global. Rend. Sem. Mat. Univ. Padova, 119 (2008), 21-95.

[Ga3] É. Gaudron. Minorations simultanées de formes linéaires de logarithmes de nombres algébriques. Bull. Soc. Math. Fr., 142 (2014), 1-62.

[Ga4] É. Gaudron. Lower bound for the Néron-Tate height (with V. Bosser). Oberwolfach Reports, workshop «Diophantische Approximationen » organisé par Y. Bugeaud, P. Habegger et U. Zannier. Report $n^{\circ} 21$, p. $22-24,2016$

[GR1] É. Gaudron et G. RÉmond. Minima, pentes et algèbre tensorielle. Israel Journal of Math., 195 (2013), $565-591$.

[GR2] É. Gaudron et G. RÉmond. Théorème des périodes et degrés minimaux d'isogénies. Commentarii Math. Helv. 89 (2014), 343-403.

[GR3] É. Gaudron et G. Rémond. Polarisations et isogénies. Duke Math. J., 163 (2014), $2057-2108$.

[GS] H. Gillet et C. Soulé. An Arithmetic Riemann-Roch Theorem. Invent. Math., 110 (1992), $473-543$.

[Ma1] D. MASSER. Small values of heights on families of abelian varieties, in Diophantine approximation and transcendence theory (Bonn, 1985). Lecture Notes in Math. 1290, (1987), 109-148.

[Ma2] D. MAsser. Lettre à Daniel Bertrand, 17 novembre 1986.

[MaWü] D. Masser et G. Wüstholz. Periods and minimal abelian subvarieties. Ann. of Math., 137 (1993), 407458.

[MiWa] M. Mignotte et M. Waldschmidt. Linear forms in two logarithms and Schneider's method. II. Acta Arith., 53 (1989), 251-287.

[MH] J. Milnor et D. Husemoller. Symmetric bilinear forms, volume 73 de Ergebnisse der Mathematik und ihrer Grenzgebiete. Springer-Verlag, 1973. 
[Na] M. NakAmaye. Multiplicity estimates on commutative algebraic groups. J. reine angew. Math., 607 (2007), $217-235$.

[Pe] F. Pellarin. Sur la distance d'un point algébrique à l'origine dans les variétés abéliennes. J. Number Theory, 88 (2001), 241-262.

[PW] P. Philippon et M. Waldschmidt. Formes linéaires de logarithmes sur les groupes algébriques commutatifs. Illinois J. Math., 32 (1988), 281-314.

[Ré1] G. RÉmond. Conjectures uniformes sur les variétés abéliennes. Quarterly Journal of Math., 69 (2018), 459-486.

[Ré2] G. Rémond. Degré de définition des endomorphismes d'une variété abélienne. Prépublication 2017. https://www-fourier.ujf-grenoble.fr/ remond/4441.pdf

[Si] A. Silverberg. Fields of definition for homomorphisms of abelian varieties. J. Pure Appl. Algebra. 77 (1992), 253-262.

[Wi] B. Winckler. Problème de Lehmer sur les courbes elliptiques à multiplications complexes. Acta Arith., 182 (2018), 347-396

Vincent Bosser

Laboratoire de Mathématiques Nicolas Oresme

UMR 6139, Université de Caen

Boulevard Maréchal Juin

14032 Caen Cedex

France

vincent.bosser@unicaen.fr
Éric Gaudron

Université Clermont Auvergne

CNRS, LMBP

F-63000

Clermont-Ferrand

France

Eric.Gaudron@uca.fr 